Luiz Augusto Martin Gonçalves

\title{
COMPENSAÇÃO DINÂMICA EM CAMES
}

Tese apresentada à Escola de Engenharia de São Carlos da Universidade de São Paulo, como parte dos requisitos para a obtenção do Título de Doutor em Engenharia Mecânica.

Orientador: Prof. Dr. Álvaro Costa Neto

São Carlos 2007 
À toda a minha família 


\section{AGRADECIMENTOS}

Ao Prof. Dr. Álvaro Costa Neto, mais do que orientador, um companheiro e amigo de longa data.

Aos colegas de departamento, professores e funcionários, pela amizade, companheirismo e colaboração.

Ao CNPq (Conselho Nacional de Desenvolvimento Científico e Tecnológico) pela bolsa de estudos que deu suporte ao desenvolvimento da maior parte deste trabalho na Katholieke Universiteit Leuven na Bélgica.

Ao Prof. Dr. Joris De Schutter pela orientação, pelo apoio, pela compreensão e por todo o suporte durante toda minha estadia na Bélgica.

Aos professores, funcionários e colegas do PMA, departamento onde desenvolvi boa parte de meu trabalho na Bélgica.

À toda a minha família sem o suporte da qual certamente não estaria hoje concluindo este trabalho. 


\section{RESUMO}

GONÇALVES, L.A.M. (2007). Compensação Dinâmica em Cames. Tese (Doutorado) - Escola de Engenharia de São Carlos, Universidade de São Paulo, São Carlos, 2007.

Em um sistema came-seguidor a dinâmica do sistema seguidor faz com que o movimento final se desvie daquele especificado. Este efeito pode ser compensado considerando-se o modelo dinâmico inverso do sistema seguidor no projeto da lei de movimento do came. Considerando-se constante a velocidade do came, o sistema seguidor tem dinâmica linear. Entretanto, devido à razão de transmissão variável, e devido a outros efeitos não lineares, o sistema de acionamento como um todo é não linear, e procedimentos não lineares devem ser utilizados para se ajustar a lei de movimento do came. Uma análise teórica, suportada por simulações, mostra o potencial deste procedimento, ao menos no caso de uma dinâmica simples do sistema seguidor: uma considerável redução do erro de movimento, e uma boa robustez relativa a erros na freqüência de ressonância e razão de amortecimento estimadas. Experimentos com o acionamento por um servomotor sub-dimensionado, como é de se esperar, mostram resultados diferentes, devido à velocidade angular não constante. A flutuação observada na velocidade angular em torno do valor constante é então levada em consideração para o projeto da lei de movimento com nonlinear feedforward.

Palavras-chave: cames, vibração em cames, cames sintonizados, cames compensados dinamicamente, compensação por feedforward, otimização de cames. 


\section{ABSTRACT}

GONÇALVES, L.A.M. (2007). Dinamically Compensated Cams. Ph.D. Thesis Escola de Engenharia de São Carlos, Universidade de São Paulo, São Carlos, 2007.

In a cam-follower system the dynamics of the follower-train mechanism cause an actual motion which deviates from the desired one. This effect can be compensated by taking into account the inverse dynamic model of the followertrain in the design of the cam motion law. By considering a constant cam velocity, the follower-train has linear dynamics. However, due to the varying transmission ratio, and due to other nonlinear effects, the whole drive train is a nonlinear system, and nonlinear procedures should be used to fit a motion law. A theoretical analysis with only the linear feed-forward compensation, supported by simulation results, has shown the potential of this approach, at least in the case of simple follower-train dynamics: a considerable reduction of the motion error, and a good robustness with respect to errors in the estimated resonance frequency and damping ratio of the follower-train. Experiments with a small driving servomotor, as expected, show different results, due to the non-constant angular velocity. The observed cam angular velocity ripple is then taken into account to design a complete nonlinear feedforward motion law.

Key-words: Cams, cam vibration, tuned cams, dynamically compensated cams, feedforward compensation, cams optimization. 


\section{SUMÁRIO}

\section{SUMÁRIO}

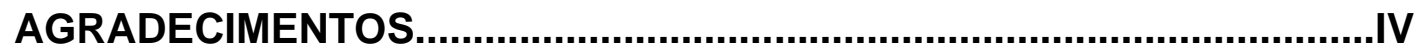

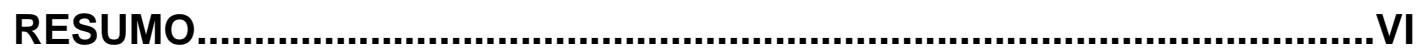

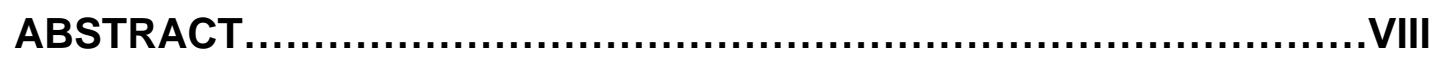

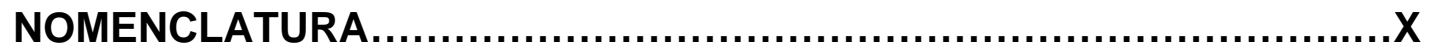

\section{Introdução}

1.1 Motivação

1.2 Objetivos

1.3 Contribuições

1.4 Estrutura da Tese

\section{Revisão da Literatura}

2.1 Algumas definições e o Desenvolvimento Inicial dos Cames

2.2 (Mais Recentes) Desenvolvimentos em Dinâmica de Sistemas Acionados por Cames 
2.3 Desenvolvimentos Relacionados com Cames Sintonizados e com Compensação Dinâmica

\section{Estudo de Caso}

3.1 Descrição do Problema

3.2 Compensação Linear (Linear Feedforward)

3.2.1 Introdução

3.2.2 Projeto da Lei de Movimento com Linear Feedforward

3.2.2.1 Modelo Dinâmico do Sistema

3.2.2.2 Princípio de Projeto da Lei de Movimento com Linear Feedforward

3.2.2.2.1 Modelo Reduzido

3.2.2.2.2 Análise de Robustez

3.2.2.3 Projeto e Implementação da Lei de Movimento com Linear Feedforward

3.2.2.2.1 Aplicação de Séries de Fourier ao Projeto da Lei de Movimento

3.2.2.2.2 Implementação da Lei de Movimento com Linear Feedforward

3.3 Compensação Não Linear (Nonlinear Feedforward)

3.3.1 Introdução

3.3.2 Projeto da Lei de Movimento com Nonlinear Feedforward

3.3.2.1 Modelo Dinâmico do Sistema

3.3.2.1.1 Modelo do Sistema de Acionamento do Came

3.3.2.2 Projeto e Implementação da Lei de Movimento com Nonlinear Feedforward

3.3.2.2.1 Determinação da Equação Diferencial Não Linear

3.3.2.2.2 Interpretação da Equação Diferencial Não Linear

3.3.2.2.3 Implementação da Lei de Movimento com Nonlinear Feedforward

3.3.3 Identificação dos Parâmetros do Sistema

\section{Resultados e Análises}


4.1 Introdução

4.2 Compensação Linear (Linear Feedforward)

4.3 Compensação Não Linear (Nonlinear Feedforward)

4.4 Conclusões

5 Conclusões Gerais e Trabalhos Futuros

\section{Bibliografia}

\section{Anexo}

A. Regularização da Velocidade por Compensação de Torque com um Mecanismo Inercial Adaptativo (Ballerina Flywheel) 


\section{Introdução}

\subsection{Motivação}

De tempos em tempos nas últimas décadas, com o advento e o rápido desenvolvimento de novos dispositivos baseados na micro-eletrônica para controle em geral, e para o controle de movimento em particular, mesmo alguns engenheiros vaticinam que os mecanismos com cames ficarão obsoletos, somente para serem rapidamente contraditados pela realidade. No final dos anos 1970, em "Where the Mechanical Way Still Makes Sense" (Brooke, 1979), algumas considerações sobre meio ambiente, robustez, prontidão, custo, interferência elétrica e manutenção foram usadas para justificar as vantagens relativas da aplicação de dispositivos mecânicos em relação aos eletrônicos em diversas aplicações. Mais recentemente ainda, em “Simple Cams Deliver No-Nonsense Motion Control” (Seitzer, 1997), e em "Motion control in product design" (Kota and Erdman, 1997), falta de flexibilidade era vista como uma das principais restrições de sistemas com cames, ainda que claramente compensadas pelas vantagens como alta velocidade de operação, simplicidade de operação, confiabilidade, eficiência energética, adequação ambiental e mais baixo custo. 
Os mesmos fatores que estão permitindo o maior emprego dos dispositivos citados, estão ao mesmo tempo permitindo a sinergia inteligente entre disciplinas antes isoladas tais como eletrônica, mecânica, controle e engenharia de computação (Chen, 1993), favorecendo o desenvolvimento de dispositivos mecatrônicos como o mecanismo híbrido com cames (Van de Straete and De Schutter, 1996), que supera a falta de flexibilidade dos sistemas convencionais com cames. Da mesma forma, a aplicação cruzada de conceitos e técnicas anteriormente confinadas a uma área às outras áreas, como é o caso da teoria de otimização, está permitindo não apenas um contínuo "ressurgimento" mas também um grande avanço em dispositivos como o mecanismo convencional de came e seguidor.

Os métodos para a síntese do movimento de cames, antes baseados em regras empíricas para o controle das derivadas de ordem mais alta do deslocamento do came, experimentaram nas últimas décadas profundas transformações. Para mencionar apenas algumas podemos citar os trabalhos com funções polinomiais objetivando sintonizar o projeto do came de forma a eliminar a vibração residual na velocidade nominal de operação (Stoddart, 1953; Chen, 1982), a extensão do mesmo procedimento para se eliminar a vibração residual agora para múltiplas velocidades nominais de operação (Kanzaki and Itao, 1972), o uso da técnica de multiplicadores de Lagrange (Wiederrich and Roth, 1975; Chew and Chuang, 1995), a aplicação da teoria de controle ótimo na síntese dinâmica (Chew, Freudenstein and Longman, 1983; Fabien, Longman and Freudenstein, 1994), e finalmente a adaptação e extensão do clássico método da Polydyne mas com a representação das curvas de deslocamento do came por curvas harmônicas de Bernstein-Bézier 
ao invés de curvas polinomiais (Srinivasan and Ge, 1998). O objetivo deste último procedimento é projetar leis de movimento de cames compensadas dinamicamente não apenas para extinguir a vibração residual na velocidade nominal de operação como também reduzir a sensibilidade a essas vibrações com variações de velocidade.

Seguindo esta tendência, e dada a sempre urgente pressão por produtividade que frequentemente requer a busca de solução de compromisso entre altas velocidades de operação e mais alta ainda confiabilidade, projetistas estão sempre enfrentando o desafio de prover novas formas de se controlar e sincronizar com mais precisão cada parte móvel das máquinas para evitar ou minimizar vibrações, ruído, e quaisquer outros efeitos colaterais devido à operação sob estas novas condições.

Com o benefício adicional das novas técnicas de análise e de projeto, consequentemente, mecanismos com cames mais rápidos e mais confiáveis parecem ter de fato um permanente lugar em muitas máquinas de embalagem, montagem, produção automática, e em muitas outras aplicações.

\subsection{Objetivos}

O presente trabalho propõe o estudo de metodologias de projeto para sistemas came-seguidor que permitam a consideração dos efeitos dinâmicos globais que afetam o funcionamento desses sistemas. Inúmeros trabalhos ao longo das últimas décadas propuseram soluções para os problemas de desvios de desempenho no comportamento desses mecanismos devidos a diferentes naturezas. Assim, muito se evoluiu no que diz respeito a desvios de natureza 
geométrica, e mesmo aos de natureza cinemática, como aqueles causados por deformações elásticas, desajustes e folgas, desgaste e irregularidades nas superfícies, saltos e perdas de contato, etc. Também no que diz respeito a desvios de natureza dinâmica como aqueles com origem nas altas inércias translacionais e rotacionais, massas, rigidezes, amortecimentos, excitações, distúrbios, freqüência de excitação, desbalanceamento de forças, etc., muitos bons resultados foram conseguidos. Uma das principais questões, neste ponto, é que essas causas que originam desvios são em geral tratadas de maneira isolada, não se obtendo uma solução global e otimizada para a correção e/ou minimização desses problemas. Assim, neste trabalho, ao se estudar metodologias que permitam a consideração dos efeitos dinâmicos globais no projeto desses sistemas, propõe-se atingir, entre outros de natureza mais geral, os seguintes objetivos específicos:

- Derivar um modelo de mecanismo came-seguidor para uma dada aplicação;

- Avaliar o efeito dinâmico do sistema completo no desempenho da trajetória do seguidor;

- Desenvolver metodologias de compensação para cames;

- Validar experimentalmente a metodologia proposta;

- Avaliar sensibilidade e robustez das compensações sintetizadas;

\subsection{Contribuições}


As principais contribuições deste trabalho são:

- Desenvolvimento de metodologia de compensação para sistemas cameseguidor aplicando-se técnicas de dinâmica de sistemas, como o feedforward control;

- Desenvolvimento de metodologia para a obtenção do perfil otimizado do came com a consideração dos efeitos dinâmicos globais em seu projeto;

\subsection{Estrutura da Tese}

Incluindo a Introdução, apresentada no Capítulo 1, esta tese se divide em 5 capítulos, abrangendo a motivação do trabalho, a revisão da literatura, a proposta de projeto da lei de movimento do came com a utilização de compensação por linear feedforward, inicialmente com um modelo dinâmico reduzido e depois com um modelo dinâmico completo, bem como a proposta de projeto por compensação por nonlinear feedforward, resultados e análises de ambas as proposições, conclusões e trabalhos futuros.

O Capítulo 2 apresenta uma revisão de literatura tanto no que diz respeito aos primeiros estágios de desenvolvimento dos cames como também aos avanços mais recentes no que diz respeito aos cames sintonizados e dinamicamente compensados, e que se constituem na base para o trabalho aqui proposto.

O Capítulo 3 apresenta a idéia central dos novos métodos propostos para a compensação dinâmica dos cames utilizando-se entre outras, técnicas derivadas da área de controle automático. Nesse capítulo é detalhado o 
emprego de feedforward, técnica mais comumente associada a controle, no projeto da lei de movimento de um came sujeito a condições críticas de utilização, considerando-se entretanto dois casos distintos: no primeiro caso, trata-se apenas com a compensação da dinâmica do subsistema seguidor, enquanto que no segundo caso apresenta-se a utilização ainda da técnica de controle feedforward no projeto da lei de movimento de um came submetido às mesmas condições críticas de utilização, porém buscando agora a compensação da dinâmica do sistema como um todo. Dadas as não linearidades apresentadas pelo conjunto de acionamento do came, do próprio came, e do subsistema seguidor, aplica-se aqui a técnica de controle por nonlinear feedforward.

O Capítulo 4 apresenta os resultados e as análises relativas aos dois casos estudados ou seja, a compensação com linear feedforward e a compensação com nonlinear feedforward.

O Capítulo 5 apresenta as conclusões gerais e as sugestões de trabalhos futuros.

\section{Revisão da Literatura}

\subsection{Algumas Definições e o Desenvolvimento Inicial dos Cames}


Came, elemento mecânico de contorno especial não regular que através de interação por contato direto com outros elementos produz um movimento prescrito, tem sido utilizado já há muitos séculos. Sua evolução segue aquela dos mecanismos em geral, cuja história poderia ser rastreada desde os primórdios do desenvolvimento tecnológico. Um dos primeiros campos de aplicação de cames de uma maneira mais sistemática, entretanto, foi provavelmente na indústria holandesa de moinhos a vento no século dezessete, onde sua utilização era bem difundida (Koster, 1974).

A definição acima, embora excluindo engrenagens devido à sua forma de contorno regular, é abrangente o suficiente para incluir alguns mecanismos que de outra forma seriam excluídos, mas que são comumente aceitos com algumas restrições na definição. Duas entre estas definições são as que se seguem:

i) "um came é um componente mecânico de uma máquina que é usado para transmitir movimento para outro componente, chamado seguidor, seguindo um programa de movimento prescrito, e através de contato direto" (Chen, 1982);

ii) "um came é um componente com um perfil ou superfície curva que impõe um deslocamento por contato por ponto ou linha ao elemento seguidor" (Bögelsack, Gierse, Oravský, Prentis, and Rossi, 1983);

Estas últimas duas definições, enquanto práticas e apropriadas para a grande maioria dos assim chamados mecanismos de came e seguidor, são 
restritivas no sentido de que implicam no came como sendo o elemento motor, que transmite ou impõe movimento a um seguidor assim guiado. Este escopo também não abrange, entre outros, alguns mecanismos nos quais o contorno especial na forma é parte do elemento movido. Este é o caso, por exemplo, do mecanismo no qual um elemento oscilante com uma forma de contorno especial com um sulco onde se encaixa uma manivela de raio constante para produzir um movimento prescrito em algum ponto ou pontos do então às vezes chamado came-seguidor ou ainda mais usualmente chamado de seguidor com perfil inserido (Hunt, K. H., 1973, and Dhande, S. G. and J. Chakraborty, 1976). Para se dar a esta definição algum grau de compatibilidade com as definições clássicas, este tipo de arranjo é também conhecido como um came invertido, onde os papeis desempenhados pelo came e pelo seguidor em termos de elementos motor e movido são invertidos (Rothbart, H. A., 1956, and Mabie and Reinholtz, 1987).

A lei de movimento do came, que corresponde ao perfil imposto ao came, foi por muito tempo projetada com base apenas em considerações cinemáticas. Como as velocidades envolvidas não eram muito elevadas, o movimento final desejado do mecanismo de came e seguidor guardava muitas vezes uma relação próxima com aquele movimento prescrito no perfil do came. Mesmo no primeiro quarto do século passado, quando cames eram já extensivamente utilizados em diversos campos de aplicação, pouca ou nenhuma atenção era observada aos aspectos dinâmicos desses mecanismos (Koster, 1974).

Apenas com o início do aparecimento de falhas mecânicas em algumas unidades de válvulas de automóveis e aviões, como conseqüência do aumento da velocidade dos motores no início dos anos 1930, é que se iniciaram então 
os primeiros estudos objetivando evitar e minimizar vibrações ressonantes. Durante o período seguinte entretanto apenas uns poucos trabalhos foram publicados a respeito desse assunto e apenas no início dos anos 1950 é que estudos mais sistemáticos sobre as características dinâmicas dos sistemas acionados por cames começaram a ser elaborados (Chen, 1977). Esta tendência no campo dos mecanismos acionados por cames não era muito diferente em relação ao que acontecia com relação ao campo dos mecanismos em geral, no qual uma das primeiras mais sérias tentativas para se organizar a nomenclatura dos aspectos de dinâmica em mecanismos foi apresentada somente no início dos anos 1970 (Erdman and Sandor, 1972). Em seu trabalho de revisão "Kineto-Elastodynamics - A Review of the State of the Art and Trends", estes autores reconheceram o rápido crescimento da importância da necessidade de se considerar a inerente elasticidade de mecanismos em aplicações de alta velocidade, e ofereceram diversas novas definições para a terminologia desta área. $\mathrm{Na}$ maioria da literatura relacionada a sistemas acionados por cames, entretanto, continua ainda a prevalecer uma terminologia básica e geral que classifica a maioria dos fatores envolvidos em um dos dois principais grupos de características cinemáticas e dinâmicas dos mecanismos.

Os primeiros procedimentos para o projeto das leis de movimento dos cames, embora com diversos aperfeiçoamentos ao longo do tempo, poderiam na maior parte do século passado ser classificados em um dos seguintes dois métodos: "(a) assumir o movimento requerido para o elemento seguidor e então projetar o came para produzir esse movimento, ou (b) assumir a forma do came e determinar quais características de deslocamento, velocidade e aceleração esse contorno produziria" (Mabie and Reinholtz, 1987). O primeiro 
método poderia ainda ser aplicado utilizando-se procedimentos gráficos ou algumas vezes analíticos, que é o que ainda se encontra na maioria dos livros gerais sobre máquinas e mecanismos (Mabie and Reinholtz, 1987, Burton, P., 1979, and Shigley, 1961, por exemplo) e também em livros mais específicos sobre cames (Neklutin, 1969, Molian, 1968, and Jensen, 1965, por exemplo). Um dos principais problemas com o primeiro método foi, durante um longo período de tempo, as dificuldades envolvidas na manufatura do perfil do came resultante daqueles procedimentos gráficos ou analíticos. O segundo método, nas aplicações em que era apropriado ou aceitável, poderia superar este problema pela utilização de perfis de cames mais fáceis de se produzir de forma mais precisa e barata. Exemplos deste último tipo são alguns dos cames utilizados em motores a combustão interna (Rothbart, H. A., 1956).

Com exceção de uns poucos mecanismos de came e seguidor onde a totalidade da trajetória do elemento final do sistema seguidor é completamente especificada, a grande maioria destes mecanismos tem por objetivo fazer com que o elemento final do sistema seguidor passe por certos pontos de controle com características de movimento prescritas. Isto, em princípio, dá ao projetista grande liberdade de escolha, por exemplo, para definir como tem um seguidor que se mover para atingir aqueles pontos-alvo com os desejados atributos estabelecidos para o elemento final do seguidor. Como as restrições são estabelecidas somente para alguns pontos, as diferentes formas de se fazer o elemento final do seguidor se mover entre dois desses pontos prescritos deram margem ao longo do tempo ao surgimento de diversas curvas para os perfis dos cames. Dependendo da aplicação específica, cada curva poderia apresentar algumas vantagens e algumas desvantagens. Desta forma, e 
durante diversas décadas do desenvolvimento dos cames, a evolução ficou então centrada principalmente na proposição de novas curvas e na subseqüente comparação de seus atributos com os das outras já existentes.

Como o came tem um movimento cíclico, o seguidor pode executar durante cada um destes ciclos movimentos particulares consistindo em eventos de elevações, permanência em regimes constantes, e retornos. "Elevação é o movimento do seguidor ao se afastar do centro do came; permanência em regime constante é o movimento do sistema no qual o seguidor fica em repouso; e retorno é o movimento do seguidor em direção ao centro do came." (Chen, 1982). Os primeiros projetos de curvas para a elevação e o retorno tiveram início então com curvas simples baseadas em polinômios algébricos e curvas trigonométricas. As curvas simples baseadas em polinômios algébricos incluem a de velocidade constante, a de velocidade constante modificada com arcos circulares, a de aceleração constante também conhecida como parabólica, as curvas distorcidas de aceleração, etc. As curvas trigonométricas, entre outras, incluem as de harmônica simples, a cicloidal, e as curvas elípticas. Seguindo este desenvolvimento, aparecem então as curvas polinomiais com derivadas de ordem mais altas, as harmônicas atenuadas, as curvas trigonométricas modificadas, e ainda diversas outras, incluindo curvas que são ou modificações ou combinações de características das anteriores.

Durante a maior parte deste período inicial, entretanto, e mesmo bem depois do inicio dos anos 1950 quando as características dinâmicas dos sistemas acionados por cames começaram a ser estudadas de forma mais sistematizada, a tendência geral neste campo se parecia mais com a tentativa de se obter uma receita universal para o "melhor perfil de came de todos os 
tempos" o qual apresentaria então as melhores respostas para as mais diversas aplicações. Assim, diversos estudos comparando as curvas disponíveis foram apresentados, em que algumas características particulares de alguma das curvas disponíveis eram escolhidas para se concluir que essa era então a curva mais apropriada para se produzir os melhores resultados. De fato, não era muito difícil durante esse período encontrar afirmações aparentemente contraditórias entre diferentes pesquisadores, mas isto era em geral somente o resultado das tentativas para se generalizar as conclusões. Dado o grande numero de variáveis envolvidas na análise de um sistema completo de came e seguidor, e devido às dificuldades envolvendo as interações entre essas diversas variáveis, a maior parte dos trabalhos era realizada com base em diversas hipóteses simplificadoras de forma a simplificar as análises. Como essas hipóteses não eram em geral as mesmas, também diferentes terminariam sendo as resultantes conclusões. Em sua maioria, o resultado do trabalho realizado ao longo desse período poderia então ser resumido a regras empíricas, geralmente relacionadas com 0 controle das derivadas das curvas de deslocamento dos cames. A enorme quantidade de literatura cobrindo o trabalho feito nesse período em mecanismos acionados por cames é de certa forma um atestado da importância desses sistemas na indústria, o que pode ser também verificado por extensivas revisões disponíveis sobre esse assunto (Chen, 1977, Chen, 1982, and Norton, 1993, entre outros). 


\section{2 (Mais Recentes) Desenvolvimentos em Dinâmica de Sistemas Acionados por Cames}

Os estudos das características dinâmicas de sistemas acionados por cames que foram conduzidos de forma mais regular depois do início dos anos 1950, assim como os avanços na tecnologia relacionada à manufatura dos cames e de protótipos de testes desses sistemas sob condições de altas velocidades de operação, em definitivo mostraram que alguns dos mais importantes efeitos nas características de sua resposta como taxa de desgaste, nível de ruído e vida útil eram derivados não somente do perfil do came mas também da resiliência do sistema seguidor.

Sem contar o grande número de trabalhos sendo desenvolvidos desde então e direcionados a atributos particulares de sistemas acionados por cames e suas respectivas aplicações específicas, um número crescente de pesquisadores começaram a atentar para as características globais do sistema came e seguidor que poderiam ter influência na resposta do elemento final do sistema seguidor. Depois de quase três décadas de pesquisas relatadas neste campo, dois grupos básicos de causas foram então sistematicamente identificadas como fontes do mau funcionamento dinâmico desses sistemas quando operando a velocidades mais altas: desvios em relação ao desempenho de origem cinemática, e aumento do carregamento estrutural (Chen, 1977). Entre as causas dos desvios de desempenho de origem cinemática podem ser notados deformações elásticas, desajustes e folgas, desgaste e irregularidades nas superfícies, saltos e perdas de contato, etc. $\bigcirc$ aumento do carregamento estrutural é principalmente relacionado às inércias 
translacionais e rotacionais, massas, rigidezes, amortecimentos, excitações e distúrbios, freqüência de excitação, desbalanceamento de forças, lubrificação das superfícies, etc. Alguns autores (Choubey and Rao, 1981) trabalharam simultaneamente com ambos os grupos de causas de desvios de desempenho que afetam a saída real dos mecanismos acionados por cames e classificaramnas como erros mecânicos e erros de flexibilidade. Outros (Kim and Newcombe, 1982) classificaram as causas dos erros em uma das três seguintes categorias: efeitos de natureza geométrica, cinemática, e dinâmica.

Em “Cam Dynamics of High-speed Systems” (Rothbart, 1956), os vários aspectos do desempenho de sistemas operando a altas velocidades foram discutidos, onde diversos fatores que influenciam na operação dos sistemas de came e seguidor são analisados, e recomendações são apresentadas como sendo a solução efetiva dos problemas de projeto. Entre diversas fontes de vibração, são apresentadas as características de aceleração, da derivada da aceleração, do impacto das cargas, considerações de balanceamento, as limitações de produção naquele momento, etc., e um guia de projeto é proposto. O efeito dos erros nos perfis na dinâmica dos cames também foi analisado (Johnson, 1957a) com um método analítico para se prever a influência de variações dimensionais sobre a velocidade e a aceleração, seguindo trabalho do mesmo autor a respeito da minimização da pressão de contato (Johnson, 1956a) e da variação da força de contato no came, identificada como a fonte das deformações elásticas que causam movimentos vibratórios (Johnson, 1956b). Johnson, que tinha já apresentado uma aplicação do método das diferenças finitas para prover técnicas de projeto de cames a partir de um ponto de vista puramente cinemático (Johnson, 1955, 1956c, 
1957b), estendeu o mesmo procedimento para a análise dinâmica e o projeto de mecanismos com cames levando-se em conta sua flexibilidade (Johnson, 1959), assumindo para isso um modelo simples anteriormente proposto para a deformação elástica da região de contato entre as superfícies do came e do seguidor Johnson, 1958).

Um procedimento de análise foi desenvolvido para se determinar os erros cinemáticos característicos na saída devidos ao processo de manufatura e montagem (Dhande S. G. and J. Chakraborty, 1975), do ponto de vista da teoria das probabilidades, e um procedimento de síntese foi proposto e discutido para a distribuição das tolerâncias em diferentes membros dos mecanismos para se obter um erro máximo de saída especificado.

Di Benedetto (Di Benedetto, 1975) desenvolveu três métodos para a síntese cinemática de perfis de cames para padrões prescritos de derivadas da aceleração: o primeiro deles baseado no método das diferenças finitas anteriormente desenvolvido por R.C. Johnson, e posteriormente aperfeiçoado por Chen (Chen, 1972, 1973), e os outros dois deduzidos considerando-se concorrentemente diferenças finitas e esquemas de integração numérica.

Di Benedetto (Di Benedetto, 1979) desenvolveu subsequentemente um algoritmo para a síntese de perfis de cames em função de uma aceleração préestabelecida do elemento seguidor.

Johnson (Johnson, 1959) desenvolveu uma análise e uma metodologia de projeto para mecanismos com cames com relativa flexibilidade considerando mais de um grau de liberdade.

O trabalho de Kim e Newcomb (Kim \& Newcomb, 1982) produziu resultados a partir de simulações feitas para se investigar os efeitos de erros de 
manufatura nas superficies de contato de mecanismos com cames em paralelo com todos os possíveis erros devidos à flexibilidade desses mecanismos. Um modelo dinâmico com onze graus de liberdade de um sistema de came e seguidor incluindo o subsistema de acionamento foi desenvolvido, e a simulação dinâmica foi combinada com uma simulação estocástica da natureza aleatória das tolerâncias de usinagem que tinham um considerável efeito na saída real. Fatores como perda de contato, pré-carregamento da mola retentora, variações na velocidade angular, características das várias curvas de movimento, flexibilidade dos vários componentes, não circularidade dos rolamentos no eixo do came, não concentricidade do círculo de base e tolerâncias de usinagem tanto no perfil do came como no do seguidor foram considerados, e um método analítico para se calcular a constante de mola equivalente entre o came e o seguidor considerando-se contato Hertziano foi desenvolvido. Os efeitos da tolerância e erros de flexibilidade foram examinados tanto separadamente como em combinação. Uma revisão de assuntos relacionados foi também apresentada, onde os fatores afetando a saída de movimento de um sistema de came e seguidor são classificados em três categorias como erros geométricos, erros cinemáticos, e erros devidos a efeitos dinâmicos. Os autores enfatizam ainda que como nenhum trabalho anterior havia considerado todas estas três fontes de erro simultaneamente, eles assim o fizeram com o recurso de simulações.

Seu principal objetivo era então divisar novas estratégias de projeto que pudessem levar a melhorias no comportamento dinâmico dos sistemas com came e seguidor. A base fundamental desses trabalhos foi a introdução da flexibilidade nos sistemas de cames em geral, e no subsistema seguidor em 
particular. A primeira fase desse período foi caracterizada também, entre outros fatores, pelas considerações sobre as tolerâncias de manufatura.

\subsection{Desenvolvimentos Relacionados com Cames Sintonizados e com Compensação Dinâmica}

Os métodos para a síntese do movimento dos cames, previamente baseado em regras empíricas e no controle das derivadas de ordem mais alta do deslocamento do came, experimentaram nas últimas décadas profundas transformações. Para mencionar apenas alguns se devem citar os trabalhos com funções polinomiais tendo como objetivo um ajuste, uma sintonização do came, para eliminar a vibração residual a uma determinada velocidade (Stoddart, 1953; Chen, 1982), a extensão do mesmo procedimento para se eliminar a vibração residual mas agora para múltiplas velocidades préestabelecidas (Kanzaki and Itao, 1972), o uso da técnica dos multiplicadores de Lagrange (Wiederrich and Roth, 1975; Chew and Chuang, 1995), a aplicação da teoria de controle ótimo para a síntese dinâmica de cames (Chew, Freudenstein and Longman, 1983; Fabien, Longman and Freudenstein, 1994), e finalmente uma adaptação e extensão do método clássido Polydyne mas com a representação das curvas de deslocamento do came por curvas harmônicas de Bernstein-Bézier ao invés de curvas polinomiais (Srinivasan and Ge, 1998). O objetivo deste último procedimento é projetar leis de movimento de cames dinamicamente compensadas não somente para se eliminar a vibração residual na velocidade nominal de trabalho mas também reduzir a sensibilidade dessas vibrações à variações de velocidade. 
Novos métodos foram também apresentados para o projeto de cames para operação em altas velocidades (Wiederrich \& Roth, 1975), tendo como principais vantagens $\mathrm{o}$ fato de assegurar a acuracidade do modelo matemático assumido ao mesmo tempo em que permitia bom desempenho dinâmico. As soluções obtidas utilizando-se estes métodos mostraram a existência de vibrações de baixa intensidade que de certa forma violavam a crença comum em projeto que supunha ser necessário eliminar ou minimizar a derivada da aceleração (Jerk) para se obter um movimento com baixa vibração. Nesse artigo os autores determinam as limitações teóricas do modelo matemático até então mais comumente utilizado e estabelecem a influência das derivadas de ordem mais alta na resposta, concluindo que através do controle do conteúdo harmônico dos segmentos do came podese não somente reduzir a influência das derivadas de ordem mais alta (que são, de fato, difíceis de controlar devido às limitações de usinagem), mas também assegurar a acuracidade da resposta prevista pelo modelo assumido. Em vista desses fatos os autores apresentam novos métodos para o projeto de perfis de cames baseados em series trigonométricas finitas. O primeiro método proposto (Projeto de Came Sintonizado) se aplica àqueles casos em que o came opera a velocidades constantes ou quase constantes, quando é então teoricamente possível se controlar completamente o movimento do seguidor pelo uso desses cames sintonizados. O segundo método proposto (Projeto de Cames Baseado em Minimização do Erro Médio Quadrático) se aplica àqueles cames previstos para operar com baixo nível de vibração em uma grande gama de 
velocidades de operação (um dos principais objetivos desses métodos é a eliminação de todas as restrições possíveis de forma a se obter a maior família possível de soluções).

Um grande passo adiante nessa direção de se eliminar ou ao menos minimizar as vibrações em sistemas operando em altas velocidades foi dado por um novo método também baseado em series trigonométricas finitas (Van den Noortgate \& De Fraine, 1977), mas agora buscando evitar a excitação da estrutura da máquina através dessas séries. O método se utiliza de um número limitado de harmônicas criteriosamente selecionadas para se evitar a excitação das freqüências naturais da estrutura. Dois tipos de condições são impostas nesse caso: condições restritivas (por exemplo, um dado deslocamento a uma dada posição angular), e condições de otimização (por exemplo, minimização do erro de movimento durante o repouso do seguidor).

Outro trabalho de síntese da lei de movimento do came usando séries trigonométricas finitas foi desenvolvido por Weber (Weber, 1979) Foi então desenvolvida o que o autor denominou por síntese por Fourier para gerar curvas com quaisquer inclinações finais, de forma a conectar duas outras curvas dadas. $\mathrm{O}$ contorno resultante aciona o seguidor de forma mais suave devido à supressão das harmônicas mais altas. São apresentadas equações de Fourier para o deslocamento, velocidade, aceleração e derivadas de ordem mais alta.

De Fraine, que já havia proposto a utilização de séries trigonométricas para se evitar a excitação da estrutura da máquina, compila agora as melhores técnicas desenvolvidas em um novo trabalho (De Fraine, 1979), onde busca a 
integração das técnicas de CAD e de CAM em três programas computacionais com o objetivo de melhorar tanto o projeto como a manufatura dos cames.

Um compreensivo estudo experimental é também apresentado (Hsu et al, 1995), onde são verificados os efeitos das velocidades do came nos sistemas de came e seguidor. 


\section{Estudo de Caso}

\subsection{Descrição do Problema}

Em um sistema constituído de um came, seu sistema de acionamento, e o respectivo subsistema seguidor, a dinâmica do subsistema seguidor impõe à carga um movimento real que se desvia daquele desejado (Figuras 1a e 1b).
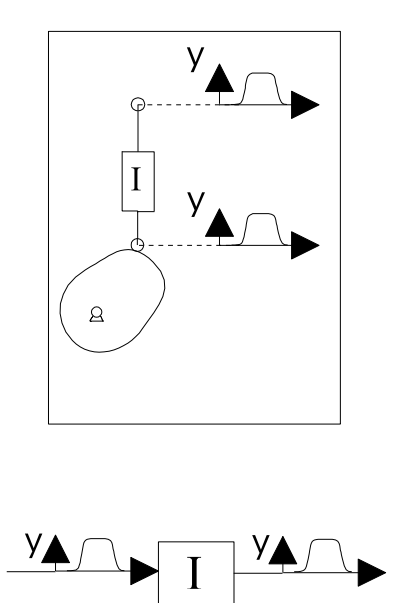

(a)
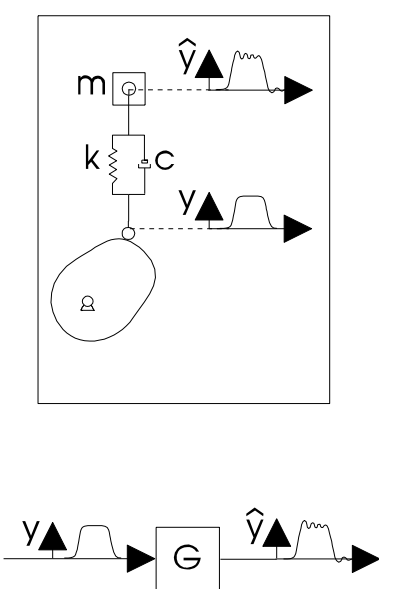

(b)

Figura 1 - Resposta do seguidor sem efeitos dinâmicos (a);

e com efeitos dinâmicos (b) 
Ao longo das últimas décadas, desde que a demanda por sucessivos aumentos de produtividade impôs às máquinas velocidades de operação cada vez maiores, diversos procedimentos foram propostos para tentar contornar este problema, com distintos graus de eficácia (Chen, 1982).

Este trabalho propõe uma nova maneira de se tratar deste problema baseado em uma idéia, usualmente aplicada à sistemas de controle, ou seja, a de que tal efeito pode ser compensado levando-se em consideração o inverso do modelo da dinâmica do subsistema seguidor no projeto da nova lei de movimento para o came.

Para melhor explicar o procedimento adotado pode ser observado que o movimento desejado, que deveria ser usinado no came se não houvesse efeitos dinâmicos, deve ser modificado pela aplicação da função transferência inversa do subsistema seguidor para gerar a lei de movimento do came com compensação (Figura 2a). O came compensado poderia então acionar 0 subsistema seguidor de forma a se obter o movimento originalmente projetado e desejado para o movimento da carga (Figura 2b), assumindo-se uma velocidade angular constante.

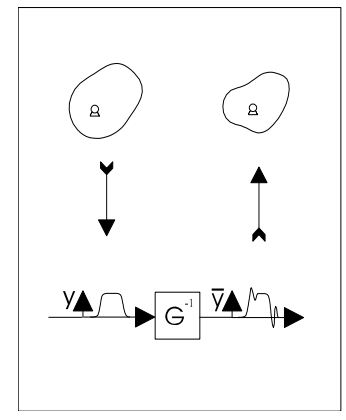

(a)

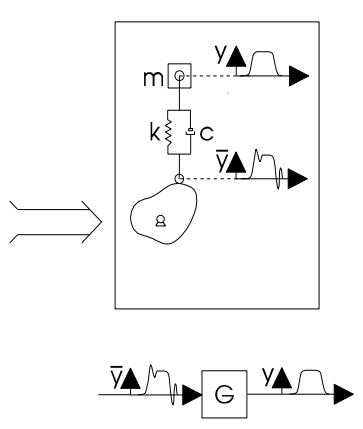

(b)

Figura 2 - Idéia básica do método proposto (a);

e resultante resposta do subsistema seguidor (b) 
Considerando-se uma velocidade angular constante, o subsistema seguidor tem uma dinâmica linear. Entretanto, devido à relação de transmissão variável, e a outros efeitos não lineares, o sistema completo é um sistema não linear, e procedimentos não lineares devem ser utilizados para se obter uma lei de movimento ideal.

No item 3.2, de forma a simplificar a explanação do método e mostrar alguns aspectos relativos à robustez do procedimento, a análise teórica que se efetua utiliza um modelo reduzido, obtido a partir do sistema não linear pela assunção de que os principais efeitos dinâmicos estão no subsistema seguidor, como se o sistema fosse de fato linear. Nesse item é descrita a obtenção de um novo perfil de came obtida com a consideração linear e com a aplicação da compensação por linear feedforward. Esse novo perfil de came é então analisado tanto em relação ao modelo reduzido como em relação ao modelo não linear completo.

Uma série inicial de experimentos com um servomotor subdimensionado, entretanto, mostra diferentes resultados, como esperado, principalmente devido à falta de potência do motor para manter a velocidade angular constante. $\mathrm{O}$ pequeno servomotor, com um controlador do tipo proporcional-integral na corrente, apresenta um efeito de "escorregamento" que é equivalente ao que se teria se a transmissão entre o motor e o came fosse flexível, como mostrado na Figura 5b. A flutuação na velocidade deve então ser levada em consideração, como mostrado no item 3.3, para se projetar a lei de movimento com a compensação por nonlinear feedforward. 


\subsection{Compensação Linear (Linear Feedforward)}

\subsubsection{Introdução}

A Figura 3 apresenta uma visão geral do banco de ensaios utilizado no desenvolvimento deste trabalho. Nessa figura podem ser vistos o servomotor que aciona o came através de uma correia dentada, o volante usado como suporte ao motor para auxiliar na manutenção da desejada velocidade angular constante, e a barra que transmite o movimento do seguidor à carga oscilante, também mostrada na figura. Em seguida tem-se os valores estimados para alguns dos parâmetros do sistema. 


\section{$\underline{\text { Test Setup }}$}

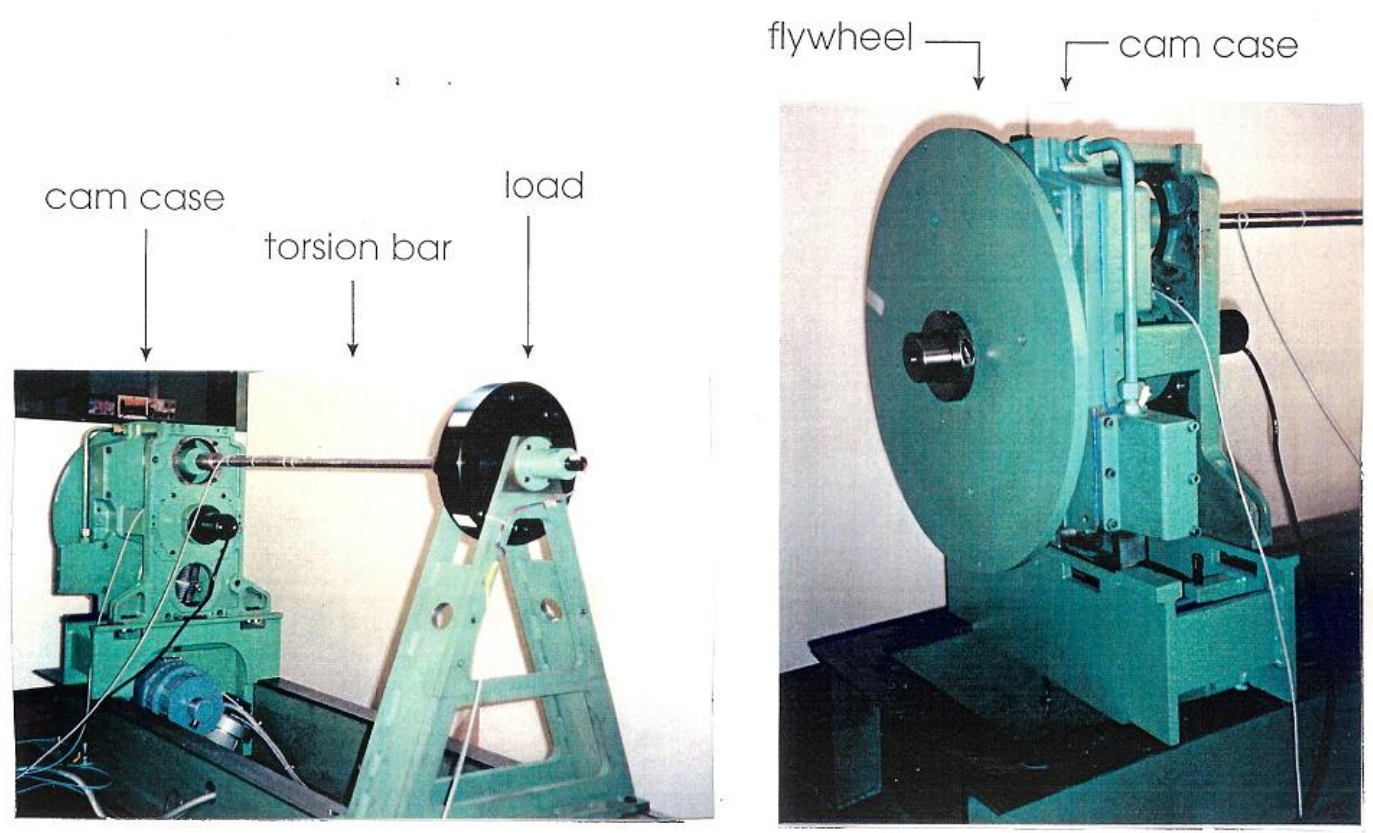

Figura 3 - Vista geral do banco de ensaios

$$
\begin{array}{ll}
\text { If }=2.08 & \text { Inércia do volante } \\
\text { IC }=0.044 & \text { Inércia do came } \\
\text { Im }=0.001 & \text { Inércia do motor } \\
\text { Ip } 0=0.0012 & \text { Inércia da polia menor } \\
\text { Ip } 1=0.0392 & \text { Inércia da polia maior } \\
\text { I1 }=\text { If }+\mathrm{IC}+\mathrm{Ip} 1+(\mathrm{Im}+\mathrm{Ip} 0) \times(75 / 32)^{\wedge} 2=2.1 \\
\mathrm{I} 2=0.126 & \text { Inércia do seguidor } \\
\mathrm{I} 3=0.357 & \text { Inércia da carga (com 4 massas) } \\
\mathrm{I3}=0.394 & \text { Inércia da carga (com 8 massas) } \\
\mathrm{C} 3=0.205 & \text { Amortecimento na carga } \\
\mathrm{K} 23=3050 & \text { Rigidez da barra torcional }
\end{array}
$$


A Figura 4 apresenta, de forma esquematizada, uma visão geral do mesmo banco de ensaios onde podem ser vistos o motor de acionamento, o came e subsistema seguidor oscilante, assim como o volante usado como suporte ao motor para auxiliar na manutenção da desejada velocidade angular constante.

\section{seguidor oscilante}

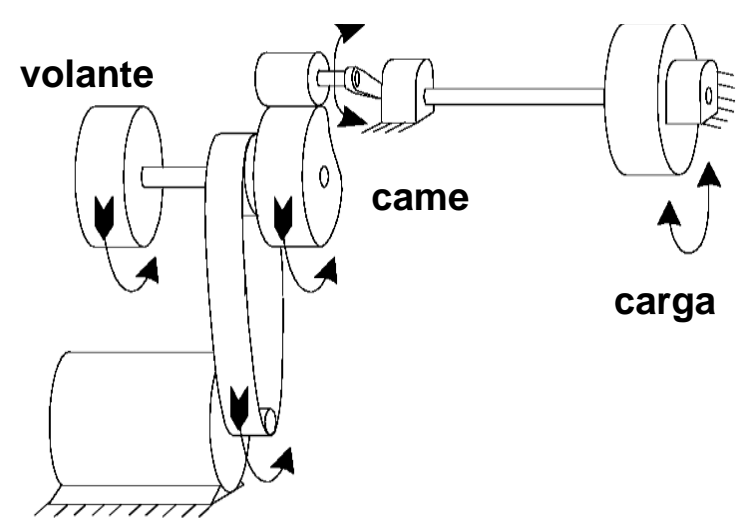

Figura 4 - Vista geral esquemática do banco de ensaios

- O dispositivo atual é do tipo master-slave, de tal forma que não ocorre a perda de contato entre o came e o seguidor;

- O perfil do came é gerado através de uma série harmônica limitada (9 componentes);

- Esta técnica é normalmente utilizada para sintetizar perfis de cames que evitem ressonâncias do sistema;

- Neste trabalho as componentes destas harmônicas serão modificadas para incluir a compensação. 


\subsubsection{Projeto da Lei de Movimento com Linear Feedforward}

\subsubsection{Modelo Dinâmico do Sistema}

As figuras (5a) e (5b) mostram dois diferentes modelos para o sistema de came e seguidor. Nelas os parâmetros relacionados ao rotor do sistema de acionamento têm índice inferior 0 , aqueles relacionados diretamente com 0 came têm índice inferior 1 , aqueles relacionados com o primeiro elemento do subsistema seguidor têm índice 2, enquanto que os parâmetros ligados ao último elemento do subsistema seguidor têm índice inferior 3. A única diferença é que para o primeiro modelo (Figura 5a), a entrada para o sistema é considerada como sendo um torque constante $\mathrm{T}_{0}$ fornecido pelo motor para $\mathrm{o}$ sistema, enquanto que para o segundo modelo (Figura 5b), assume-se que o sistema é acionado por uma velocidade angular constante $\dot{\Theta}_{0}$.

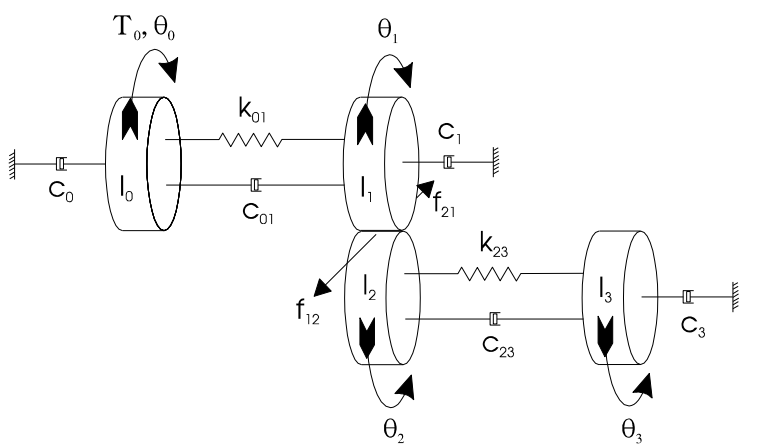

(a)

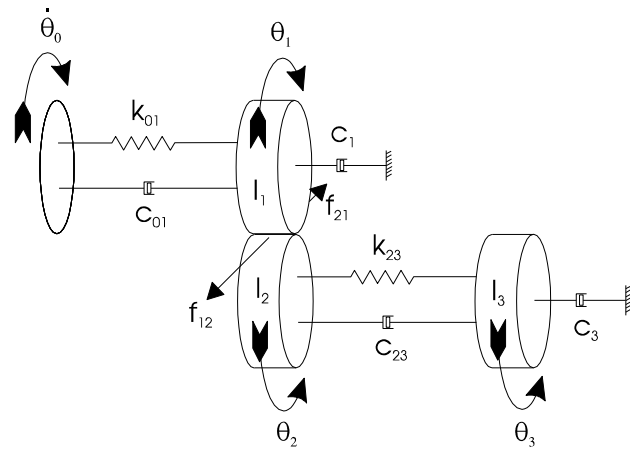

(b)

Figura 5 - Entrada Torque Constante $\mathrm{T}_{0}$ (a), e Entrada Velocidade Angular Constante $\dot{\Theta}_{0}$ (b).

As equações (3.1) e (3.2) mostram a representação em espaço de estados para os dois diferentes modelos do sistema, onde valem as seguintes relações: 


$$
\begin{array}{ll}
\theta_{2}=\mathrm{f}\left(\theta_{1}\right) & \Delta \theta_{2}=\mathrm{f}^{\prime}\left(\theta_{1}\right) \Delta \theta_{1} \\
\dot{\theta}_{2}=\mathrm{f}^{\prime}\left(\theta_{1}\right) \dot{\theta}_{1} & \mathrm{~d}\left(\Delta \theta_{2}\right) / \mathrm{dt}=\mathrm{f}^{\prime}\left(\theta_{1}\right) \mathrm{d}\left(\Delta \theta_{1}\right) / \mathrm{dt} \\
\ddot{\theta}_{2}=\mathrm{f}^{\prime}\left(\theta_{1}\right) \ddot{\theta}_{1}+\mathrm{f}^{\prime \prime}\left(\theta_{1}\right) \dot{\theta}_{1}^{2} & \mathrm{~d}^{2}\left(\Delta \theta_{2}\right) / \mathrm{dt}^{2}=\mathrm{f}^{\prime}\left(\theta_{1}\right) \mathrm{d}^{2}\left(\Delta \theta_{1}\right) / \mathrm{dt}^{2}
\end{array}
$$

Nessas relações $f$ representa a função cinemática que relaciona o seguidor (elemento 2) ao perfil do came (elemento 1), ou à lei de movimento do came propriamente dita. Também para as equações (3.1) e (3.2) valem as relações $\left\{I_{1 \mathrm{e}}=I_{1}+I_{2}\left[f^{\prime}\left(\theta_{1}\right)\right]^{2}\right\}$, a inércia equivalente para os elementos 1 e 2 , e ainda $\left\{\mathrm{W}=\left(\mathrm{m}_{1} \mathrm{e}_{1} \sin \theta_{1}+\mathrm{m}_{2} \mathrm{e}_{2} \mathrm{f}^{\prime} \operatorname{sinf} \mathrm{g}\right\}\right.$, os efeitos gravitacionais em 1 e 2 .

- Entrada Torque Constante (correspondente à figura 5a):

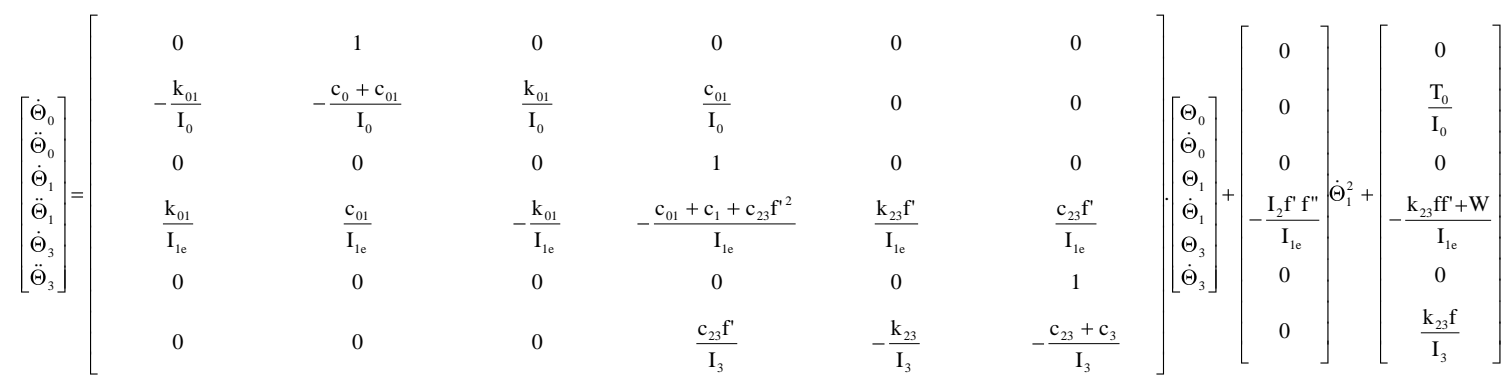

- Entrada Velocidade Angular Constante (correspondente à figura 5b):

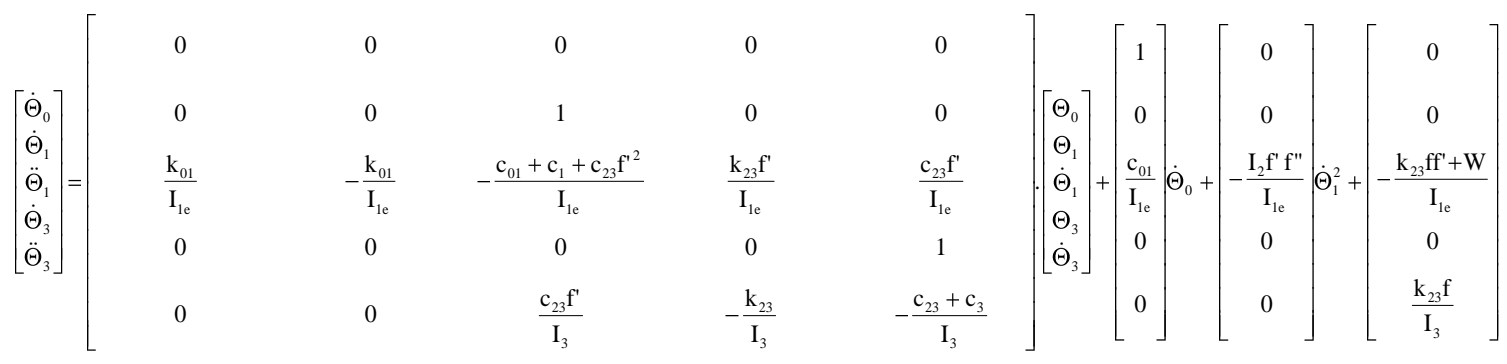


Conforme mencionado anteriormente, as dinâmicas do sistema de acionamento (motor) devem ser consideradas na modelagem do sistema completo. No primeiro dos modelos anteriormente mostrados (figura 5a), a entrada para o sistema é o torque do motor elétrico. Esse servomotor de acionamento do conjunto tinha sua corrente controlada por um controlador do tipo proporcional + integral (PI). A transmissão de movimento entre a polia do eixo do motor e a polia do eixo do came se dava através de uma correia dentada de rigidez extremamente alta, ou seja, com baixíssima flexibilidade. Observou-se, entretanto, que por se tratar de um servomotor (propositalmente) subdimensionado, nos periodos dos ciclos de movimento onde maior potência era exigida, ocorria um aparente "escorregamento" do motor, e a conseqüente queda na velocidade.

Analisando-se o conjunto pode-se verificar que essas dinâmicas podem ser substituídas por um arranjo mecânico equivalente, de mesma dinâmica. Podese mostrar que o conjunto "flexibilidade" do motor e correia de transmissão rígida pode ser substituído pelo conjunto dinamicamente equivalente velocidade de rotação constante no eixo do motor e correia de transmissão flexível.

Com base na equivalência anteriormente descrita, chega-se então ao modelo mostrado na figura (5b). Neste caso, o primeiro modelo pode ser representado pelo modelo da figura (5b). A equivalência deste último modelo com o modelo anterior é obtida através do ajuste da rigidez de acoplamento entre o acionamento do motor $\theta_{0}$ e o movimento do came $\theta_{1}$.

Este último é o modelo que será utilizado ao longo deste trabalho. 
3.2.2.2 Princípio de Projeto da Lei de Movimento com Linear Feedforward

Para descrever o procedimento para o projeto da lei de movimento com linear feedforward utiliza-se o modelo mostrado na figura (5b), onde admite-se que o motor tenha potência suficiente para impor a velocidade de rotação desejada. A partir deste modelo, e em um primeiro passo para simplificar a explanação do procedimento, admite-se inicialmente que essa rotação constante no eixo do motor seja transmitida ao eixo do came de tal forma que também neste eixo a velocidade de rotação seja mantida constante. Esta hipótese implica em que a única dinâmica relevante seria aquela do subsistema seguidor. Desta forma, obtém-se um novo modelo, mais simplificado, que será aqui tratado como modelo reduzido, e cujo equacionamento é mostrado a seguir.

\subsection{Modelo Reduzido}

Considerando-se que os principais efeitos dinâmicos estejam no subsistema seguidor, devido à sua maior flexibilidade, e para mais facilmente explicar o procedimento proposto para a geração da lei de movimento dinamicamente compensada para o came, será utilizado aqui um modelo reduzido para o sistema de came e seguidor. Neste modelo consideram-se apenas os principais efeitos dinâmicos, como mostrado na Figura (6), em que a relação entre saída e entrada é dada pela equação (3.3). Esta relação (equação 3.3) 
pode ainda ser reduzida para aquela da equação (3.4) considerando-se que o efeito de amortecimento da barra ligando as duas inércias é muito pequeno (valor estimado em torno de $0.01 \%$ ), em comparação com aquele nos mancais na inércia 3 (carga).

$$
\Theta_{3}(\mathrm{~s})=\frac{\frac{\mathrm{c}_{23}}{\mathrm{k}_{23}} \mathrm{~s}+1}{\frac{\mathrm{I}_{3}}{\mathrm{k}_{23}} \mathrm{~s}^{2}+\frac{\mathrm{c}_{3}}{\mathrm{k}_{23}} \mathrm{~s}+1} \cdot \Theta_{2}(\mathrm{~s}) \quad(3 \cdot 3)=>\Theta_{3}(\mathrm{~s})=\frac{1}{\frac{\mathrm{I}_{3}}{\mathrm{k}_{23}} \mathrm{~s}^{2}+\frac{\mathrm{c}_{3}}{\mathrm{k}_{23}} \mathrm{~s}+1} \cdot \Theta_{2}(\mathrm{~s})
$$

Como $\Theta_{2}$ expressa o movimento imposto pelo came ao seguidor, neste modelo ele representa a lei de movimento do came original, que impõe à carga um movimento $\Theta_{3}$ afetado pela dinâmica do subsistema seguidor, como mostrado na figura (1b). A nova função $\bar{\Theta}_{2}$ que resulta da aplicação da função transferência inversa $G^{-1}$ do subsistema seguidor na função original $\Theta_{2}$, como na equação (3.5), irá então produzir uma nova $\bar{\Theta}_{3}$, equação (3.6), exatamente igual à função original $\Theta_{2}$. Este procedimento é similar ao mostrado anteriormente na figura (2).

$$
\bar{\Theta}_{2}=G^{-1} \cdot \Theta_{2} \quad(3.5) \quad \Rightarrow \quad \bar{\Theta}_{3}=G \cdot\left(\bar{\Theta}_{2}\right)=G \cdot\left(G^{-1} \cdot \Theta_{2}{ }^{-}=\Theta_{2}\right.
$$

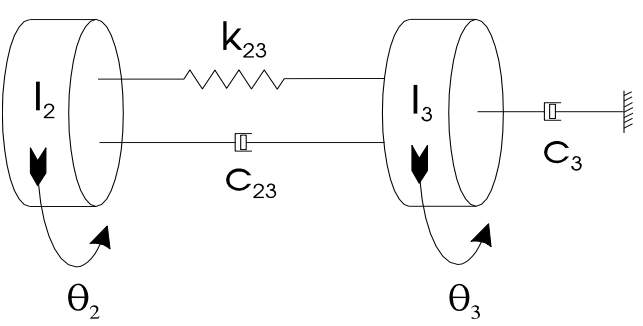

Figura 6: Modelo reduzido considerando apenas a dinâmica do seguidor 
O mesmo procedimento pode ser visto graficamente na figura (7), onde se mostra a aplicação da resposta em freqüência inversa do sistema seguidor à função $\Theta_{2}$, para se produzir uma nova função $\bar{\Theta}_{2}$. Esta nova função, quando aplicada à resposta em freqüência do sistema seguidor, irá então produzir uma função $\bar{\Theta}_{3}$, que é idêntica à função original $\Theta_{2}$.
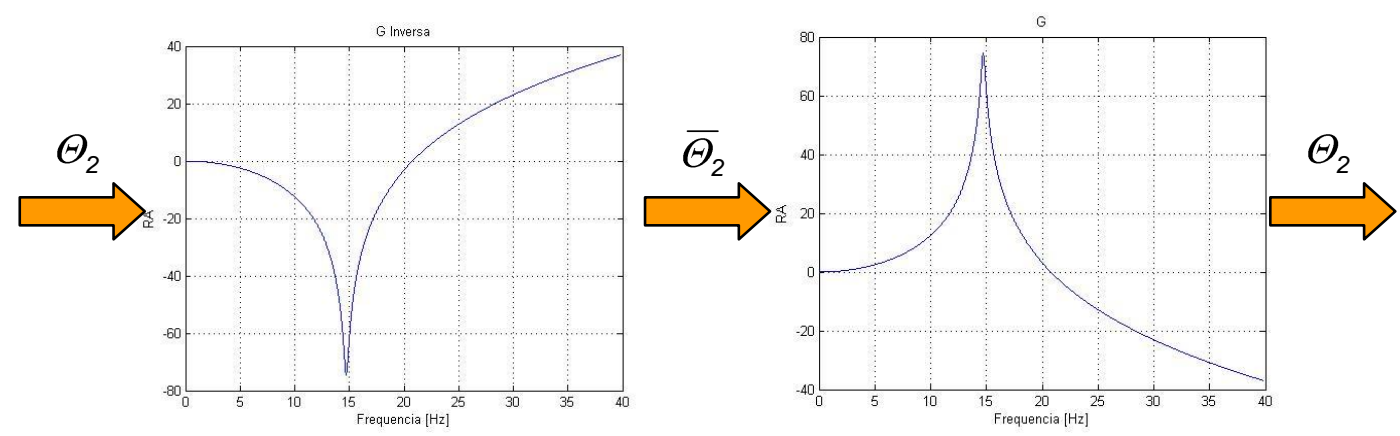

Figura 7 - Compensaçao linear considerando apenas a dinâmica do seguidor

\subsection{Análise de Robustez}

Para a verificação dos aspectos relacionados à robustez deste procedimento com respeito a erros nos valores estimados de amortecimento e da freqüência natural, bem como à possibilidade de se operar o sistema à velocidades diferentes da nominal, podemos fazer os parâmetros $\bar{\zeta}$, $\bar{\omega}$, e $\bar{\omega}_{\mathrm{n}}$ representarem respectivamente os valores reais do fator de amortecimento, a velocidade angular e a freqüência natural. Na prática, eles podem diferir dos valores estimados $\varsigma, \omega, \mathrm{e} \omega_{\mathrm{n}}$, aplicados para se ajustar a nova lei de 
movimento do came, e podem ser relacionados pelo conjunto de condições: $\left\{\bar{\zeta}=\alpha \cdot \zeta, \bar{\omega}=/ \beta \cdot{ }^{\prime} \omega, \bar{\omega}_{\mathrm{n}}=\chi \cdot \omega_{\mathrm{n}}\right\}$. Se não houver diferenças entre os valores reais e os estimados para os parâmetros, o que é equivalente a fixar $\alpha=\beta=\chi=1$ nas relações acima, o movimento resultante $\bar{\Theta}_{3}$ na equação (3.7) é exatamente igual ao $\Theta_{2}$.

$$
\bar{\Theta}_{3}=\bar{G} \cdot \boldsymbol{G}^{-1} \cdot \Theta_{2}-=\frac{\omega_{\mathrm{n}}^{2}-\omega^{2}-+2 \zeta \omega \omega_{\mathrm{n}} \mathrm{i}}{\left(\bar{\omega}_{\mathrm{n}}^{2}-\bar{\omega}^{2}\right)+2 \bar{\zeta} \bar{\omega} \omega_{\mathrm{n}} \mathrm{i}} \cdot \Theta_{2}
$$

Agora, utilizando-se da relação matemática da equação (3.8) na equação (3.7), deduz-se de forma simples a equação (3.9), que permite a verificação das verificações de robustez mencionadas.

$$
\frac{a+b i}{A+B i}=\left(\frac{A a+B b}{A^{2}+B^{2}}\right)+\left(\frac{A b-B a}{A^{2}+B^{2}}\right) \cdot i
$$

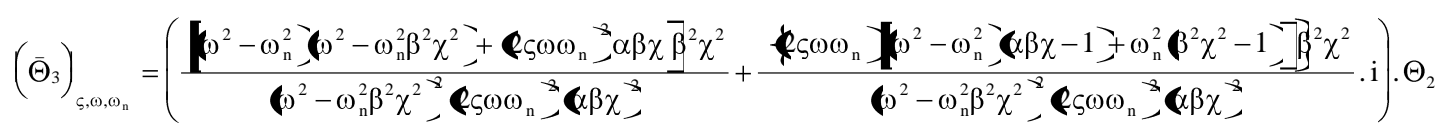

A influência da estimação incorreta dos valores dos parâmetros no comportamento do sistema pode então ser vista derivando-se da equação (3.9) equações particulares para cada um dos parâmetros, e então se atribuindo valores numéricos tanto para os valores estimados como para os reais. Para a derivação das equações particulares basta fazer a variação de apenas um parâmetro de cada vez na equação (3.9), obtendo-se assim as equações (3.10), (3.11) e (3.12). 
- Robustez em relação ao fator de amortecimento

$$
\left(\bar{\Theta}_{3}\right)_{\varsigma}=\bar{G}_{\varsigma} \cdot\left(G^{-1} \cdot \Theta_{2}\right)=\left(\left(\omega_{n}^{2}-\omega^{2}\right)+2 \varsigma \omega \omega_{n} \cdot i /\left(\omega_{n}^{2}-\omega^{2}\right)+2 \varsigma \alpha \omega \omega_{n} \cdot i\right) \cdot \Theta_{2}
$$

que, com a aplicação da relação da equação (3.8) resulta em

$$
\left(\bar{\Theta}_{3}\right)_{\varsigma}=\left(\frac{\left(\omega^{2}-\omega_{n}^{2}\right)^{2}+\left(2 \varsigma \omega \omega_{n}\right)^{2} \cdot \alpha}{\left(\omega^{2}-\omega_{n}^{2}\right)^{2}+\left(2 \varsigma \omega \omega_{n}\right)^{2} \cdot \alpha^{2}}+\frac{2 \varsigma \omega \omega_{n}\left(\omega^{2}-\omega_{n}^{2}\right)(\alpha-1)}{\left(\omega^{2}-\omega_{n}^{2}\right)^{2}+\left(2 \varsigma \omega \omega_{n}\right)^{2} \cdot \alpha^{2}} \cdot i\right) \Theta_{2}
$$

\section{- Robustez em relação à velocidade angular}

$$
\left(\bar{\Theta}_{3}\right)_{\omega}=\bar{G}_{\omega} \cdot\left(G^{-1} \cdot \Theta_{2}\right)=\left(\left(\omega_{n}^{2}-\omega^{2}\right)+2 \varsigma \omega \omega_{n} \cdot i /\left(\omega_{n}^{2}-(\omega / \beta)^{2}\right)+2 \varsigma(\omega / \beta) \omega_{n} \cdot i\right) \cdot \Theta_{2}
$$

que, com a aplicação da relação da equação (3.8) resulta em

$$
\left(\bar{\Theta}_{3}\right)_{\omega}=\left(\frac{\left[\left(\omega^{2}-\omega_{\mathrm{n}}^{2}\right)\left(\omega^{2}-\omega_{\mathrm{n}}^{2} \beta^{2}\right)+\left(2 \varsigma \omega \omega_{\mathrm{n}}\right)^{2} \cdot \beta\right] \beta^{2}}{\left(\omega^{2}-\omega_{\mathrm{n}}^{2} \beta^{2}\right)^{2}+\left(2 \zeta \omega \omega_{\mathrm{n}}\right)^{2} \cdot \beta^{2}}+\frac{\left[2 \zeta \omega \omega_{\mathrm{n}}\left(\omega^{2}+\omega_{\mathrm{n}}^{2} \beta\right)(\beta-1)\right] \beta^{2}}{\left(\omega^{2}-\omega_{\mathrm{n}}^{2} \beta^{2}\right)^{2}+\left(2 \zeta \omega \omega_{\mathrm{n}}\right)^{2} \cdot \beta^{2}} \cdot \mathrm{i}\right) \cdot \Theta_{2}
$$

- Robustez em relação à freqüência natural

$$
\left(\bar{\Theta}_{3}\right)_{\omega_{\mathrm{n}}}=\overline{\mathrm{G}}_{\omega_{\mathrm{n}}} \cdot\left(\mathrm{G}^{-1} \cdot \Theta_{2}\right)=\left(-\left(\omega_{\mathrm{n}}^{2}-\omega^{2}\right)+2 \varsigma \omega \omega_{\mathrm{n}} \cdot \mathrm{i} /\left(\left(\omega_{\mathrm{n}} \cdot \chi\right)^{2}-\omega^{2}\right)+2 \varsigma \omega \omega_{\mathrm{n}} \chi \cdot \mathrm{i}\right) \cdot \Theta_{2}
$$

que, com a aplicação da relação da equação (3.8) resulta em

$$
\left(\Theta_{3}\right)_{\omega_{\mathrm{n}}}=\left(\frac{\left[\left(\omega^{2}-\omega_{\mathrm{n}}^{2}\right)\left(\omega^{2}-\omega_{\mathrm{n}}^{2} \chi^{2}\right)+\left(2 \zeta \omega \omega_{\mathrm{n}}\right)^{2} \cdot \chi\right] \chi^{2}}{\left(\omega^{2}-\omega_{\mathrm{n}}^{2} \chi^{2}\right)^{2}+\left(2 \zeta \omega \omega_{\mathrm{n}}\right)^{2} \cdot \chi^{2}}+\frac{\left[2 \zeta \omega \omega_{\mathrm{n}}\left(\omega^{2}+\omega_{\mathrm{n}}^{2} \chi\right)(\chi-1)\right] \chi^{2}}{\left(\omega^{2}-\omega_{\mathrm{n}}^{2} \chi^{2}\right)^{2}+\left(2 \zeta \omega \omega_{\mathrm{n}}\right)^{2} \cdot \chi^{2}} \cdot \mathrm{i}\right) \cdot \Theta_{2}
$$

As figuras (8) e (9), relação de amplitudes e ângulo de fase das funções transferências nas equações (3.10), (3.11) e (3.12), mostram os efeitos causados por erros em (a), o fator de amortecimento, (b), a velocidade angular, 
e em (c), a freqüência natural, com base nos valores estimados para os parâmetros $\omega=3 \mathrm{~Hz}$ e $\omega_{\mathrm{n}}=15 \mathrm{~Hz}$.

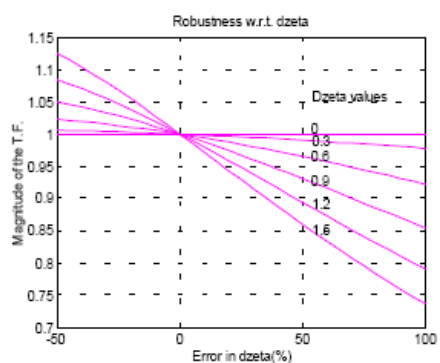

(a)

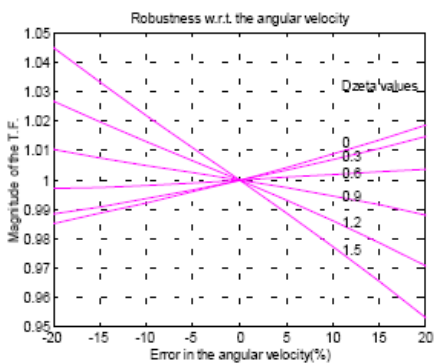

(b)

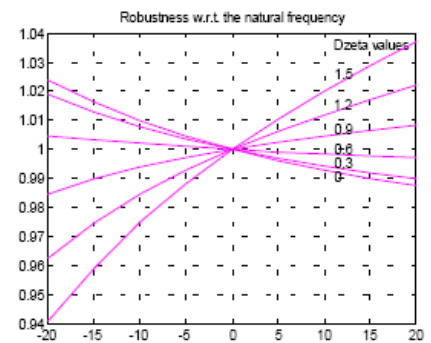

(c)

Figura 8 - Relação de Amplitudes da Função Transferência para Verificação de Robustez em Relação ao Amortecimento, Velocidade Angular e Freqüência Natural

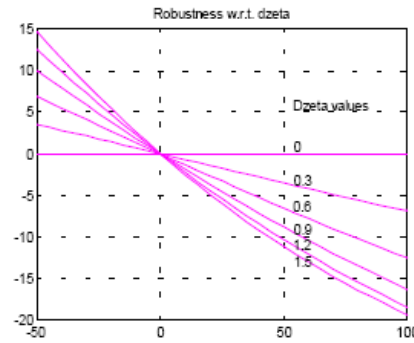

(a)

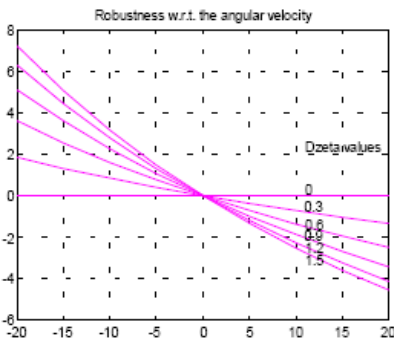

(b)

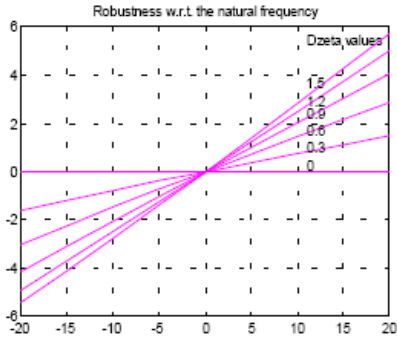

(c)

Figura 9 - Ângulo de Fase da Função Transferência para Verificação de Robustez em Relação ao Amortecimento, Velocidade Angular e Freqüência Natural

As figuras (8) e (9), com desvios desde $-50 \%$ até $+100 \%$ nos valores de amortecimento, e de $-20 \%$ até $+20 \%$ nos valores tanto da velocidade angular como da freqüência natural, mostram a robustez do presente procedimento, dentro dos limites dados pelas hipóteses de modelagem assumidas. Os 
resultados são ainda melhores para valores pequenos do fator de amortecimento. Além disso, as equações (3.11) d (3.12) apresentam a mesma relação entre $\bar{\Theta}_{3}$ e $\Theta_{2}$ e, como $\bar{\omega}=(1 / \beta)$. $\omega$ e $\bar{\omega}_{n}=\chi \cdot \omega_{n}$, disso resulta que os aspectos de robustez relativos à velocidade angular e à freqüência natural são exatamente os mesmos. A mesma conclusão poderia ser tirada a partir da equação (3.13), que mostra a influência de mudanças simultâneas na velocidade angular e na freqüência natural.

$$
\left(\Theta_{3}\right)_{\omega, \omega_{\mathrm{n}}}=\left(\frac{\left[\left(\omega^{2}-\omega_{\mathrm{n}}^{2}\right)\left(\omega^{2}-\omega_{\mathrm{n}}^{2} \beta^{2} \chi^{2}\right)+\left(2 \varsigma \omega \omega_{\mathrm{n}}\right)^{2} \cdot \beta \chi\right] \beta^{2} \chi^{2}}{\left(\omega^{2}-\omega_{\mathrm{n}}^{2} \beta^{2} \chi^{2}\right)^{2}+\left(2 \varsigma \omega \omega_{\mathrm{n}}\right)^{2} \cdot \beta^{2} \chi^{2}}+\frac{\left[2 \zeta \omega \omega_{\mathrm{n}}\left(\omega^{2}+\omega_{\mathrm{n}}^{2} \beta \chi\right)(\beta \chi-1)\right] \beta^{2} \chi^{2}}{\left(\omega^{2}-\omega_{\mathrm{n}}^{2} \beta^{2} \chi^{2}\right)^{2}+\left(2 \varsigma \omega \omega_{\mathrm{n}}\right)^{2} \cdot \beta^{2} \chi^{2}} \cdot \mathrm{i}\right) \cdot \Theta_{2}
$$

A equação (3.13) também mostra que uma mudança na freqüência natural pode compensar a mesma variação na velocidade angular, uma vez que o produto $(\boldsymbol{B} \cdot \boldsymbol{x})=\mathbf{1}$ faz a amplitude da resposta ser igual a um e a fase ser igual a zero. Inversamente, igual resultado vale também para uma mudança na velocidade angular, que compensa exatamente uma mudança similar na freqüência natural.

Os resultados mostrados acima, com o modelo reduzido e com a consideração apenas da dinâmica do subsistema seguidor, além de ilustrar de forma mais simples a idéia central deste trabalho, permitem ainda visualizar a robustez do procedimento básico adotado, ao menos naquelas condições impostas pelas hipóteses que levaram a esse modelo. Como para a implementação dessa técnica (feedforward) em um sistema real não basta considerar apenas o modelo reduzido, mas sim verificar as implicações dessa 
implementação no sistema completo, a seguir se apresenta o projeto e a implementação da lei de movimento com linear feedforward para o sistema não linear completo.

3.2.2.3 Projeto e Implementação da Lei de Movimento com Linear Feedforward

Para completar o modelo do sistema descrito na secção 3.2.2.1 de forma a possibilitar a realização de simulações e ensaios experimentais com o sistema completo, deve-se mostrar o procedimento adotado para se construir o came, baseado em séries harmônicas limitadas de Fourier.

3.2.2.2.1 Aplicação de Séries de Fourier ao Projeto da Lei de Movimento

Qualquer lei de movimento inscrita em um came através da sua usinagem pode ser representada por séries infinitas de Fourier. Para os objetivos deste trabalho foi escolhida para se gerar o perfil do came uma série de Fourier truncada, ou uma série harmônica limitada de Fourier (DE FRAINE, 1995). principal objetivo da utilização das séries harmônicas limitadas de Fourier em sistemas de came e seguidor é o de se evitar a excitação de freqüências naturais do subsistema seguidor ou de algum outro componente do conjunto, ao não se empregar no projeto aquelas harmônicas que podem coincidir com freqüências de ressonância do sistema. Outra grande vantagem do emprego das séries harmônicas é que fica assim possível uma otimização global de 
corpo rígido, ao contrário do que ocorre com os perfis de cames projetados a partir de combinações de trajetórias parciais.

No caso deste trabalho, entretanto, a escolha de séries harmônicas limitadas de Fourier para se desenvolver o perfil dos cames deveu-se a outro motivo, que não aquele de se evitar a excitação de ressonâncias do sistema. $O$ objetivo aqui foi justamente o oposto, ou seja, desejava-se que uma das harmônicas coincidisse exatamente com a freqüência natural do subsistema seguidor, de forma a enfatizar o problema a ser resolvido com os métodos aqui propostos. Assim, para uma velocidade angular de regime permanente estabelecida em $2.96 \mathrm{~Hz}$, o perfil projetado para o came nominal com séries harmônicas limitadas tem a sua $5^{\underline{a}}$ harmônica exatamente igual à freqüência natural de $14.8 \mathrm{~Hz}$ do subsistema seguidor, conforme mostrado na figura (10) abaixo.

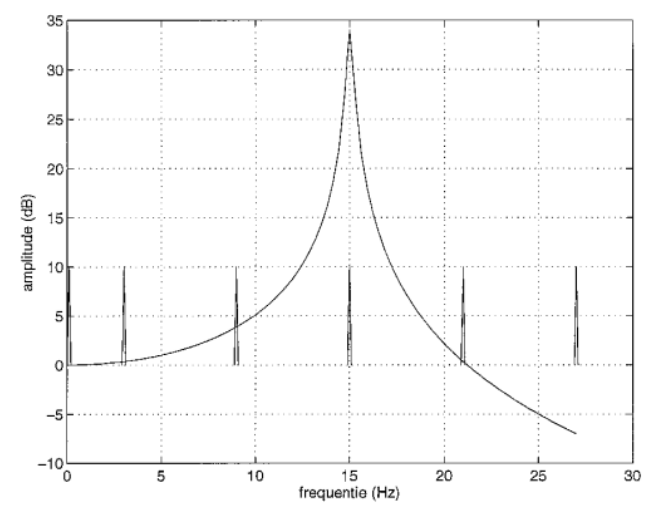

Figura 10 - Harmônica do Perfil do Came Coincidente com a Freqüência Natural do Subsistema Seguidor 
3.2.2.2.2 Implementação da Lei de Movimento com Linear Feedforward

Um modelo linear torna possível, como visto anteriormente, a obtenção da função transferência inversa exata para o sistema dinâmico e então a simulação com o modelo reduzido, com uma boa estimação dos parâmetros, claramente produz bons resultados. As figuras (8) e (9) também ilustram esta afirmação, com as relações de amplitude próximas a um e os ângulos de fase próximos à zero para pequenos desvios nos parâmetros.

Como com um modelo não linear não existe um modelo dinâmico inverso, o procedimento agora consiste em se aplicar ao came original o modelo dinâmico inverso apenas da dinâmica (linear) do subsistema seguidor, e não aquele do sistema não linear completo. As simulações e experimentos são então realizados com o sistema não linear completo, porém com apenas a compensação por linear feedforwarding da dinâmica do subsistema seguidor. Para compensar a dinâmica do sistema não linear completo devem ser usados procedimentos não lineares na geração de um novo came, mas isto é objeto do item (3.3). 


\subsection{Compensação Não Linear (Nonlinear Feedforward)}

\subsubsection{Introdução}

No item 3.2, que tratou da compensação linear, discutiu-se a aplicação do came compensado por linear feedforward em dois diferentes modelos: um primeiro modelo, reduzido, do sistema, onde se garantia a velocidade angular constante no eixo do came, e em um modelo do sistema completo, com suas não linearidades, onde a velocidade no eixo do came poderia oscilar.

Para se obter bons resultados com essa compensação por linear feedforward aplicada ao modelo completo verifica-se que as oscilações na velocidade angular não podem ser muito acentuadas. Assim, se essas oscilações forem tais que prejudiquem o bom desempenho do sistema, elas têm que ser diminuídas de alguma forma. Isto pode se dar através do emprego de um sistema de acionamento mais potente (maior servomotor), ou de um volante maior, ou mesmo com o emprego de outras técnicas tais como compensação de torque (Nishioka, 1995). Caso não seja possível ou mesmo desejável buscar essa diminuição das oscilações, e o desempenho do sistema continue prejudicado, deve-se então buscar outra solução. Disto é que trata este item, onde se propõe que a flutuação observada na velocidade angular seja levada em consideração no projeto de uma nova lei de movimento para o came, levando-se em conta agora toda a dinâmica não linear do sistema. Esta nova técnica, levando-se em conta as não linearidades do sistema completo, resulta em uma otimização para o conjunto através da obtenção de um novo 
perfil de came onde se obtém a chamada compensação por nonlinear feedforward. Como um sistema não linear não possue uma dinâmica inversa, como ocorre quando se trata de um sistema linear, neste caso técnicas de desacoplamento e de solução numérica das equações diferenciais não lineares devem então ser empregadas.

\subsubsection{Projeto da Lei de Movimento com Nonlinear Feedforward}

\subsubsection{Modelo Dinâmico do Sistema}

A figura (11) mostra o modelo físico do sistema dinâmico detalhando o sistema de acionamento, a transmissão entre o eixo do motor e o came, e o subsistema seguidor completo.

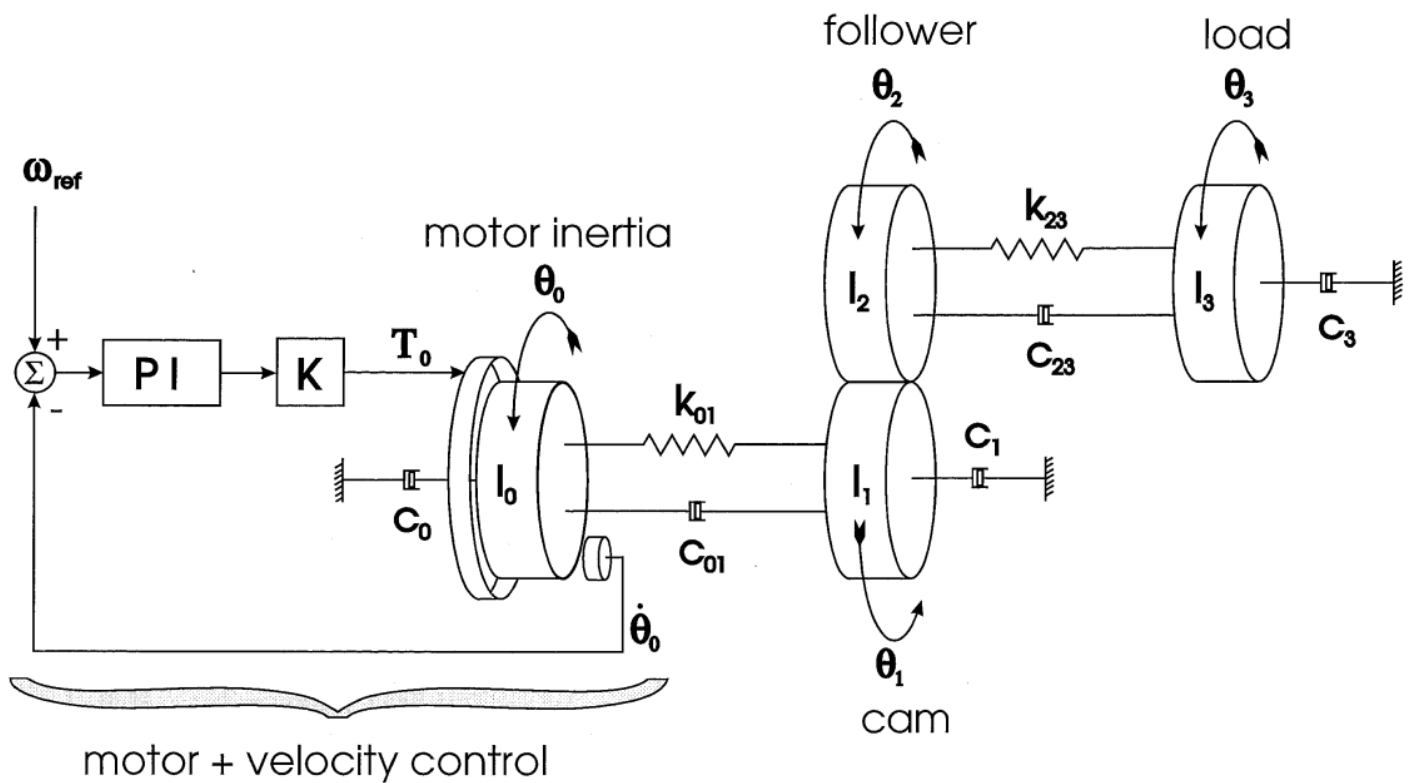

Figura 11- Modelo Físico do Sistema de Acionamento, Came e Seguidor 


\subsection{Modelo do Sistema de Acionamento do Came}

O sistema de acionamento do came é composto de um servomotor com controle proporcional + integral $(\mathrm{PI})$ na velocidade angular, como pode ser visto na figura (11). Ao se projetar o banco de ensaios, e visando acentuar o problema proposto de compensação dinâmica do sistema, esse motor teve a sua potência propositalmente subdimensionada, o que conta favoravelmente em termos de redução de custos. Ao se estudar o problema em questão notouse que em certas condições, mantendo-se constante o torque do motor, ele não conseguia manter constante a velocidade de rotação. A transmissão de movimento entre o eixo do motor e o eixo do came era feita através de uma correia dentada de rigidez extremamente alta. Concluiu-se que esse efeito de "escorregamento" do motor em certas partes do ciclo de movimento era equivalente a se ter um motor mais potente, que mantivesse constante sua velocidade angular, e ter uma transmissão flexível entre o eixo do motor e o came. Assim, como mostrado no item 3.2, com o devido ajuste na flexibilidade da correia no modelo matemático, o modelo inicialmente adotado com entrada torque constante pode ser substituído por um modelo com entrada velocidade angular constante no eixo do motor e flexibilidade na transmissão. Assim, o modelo anterior pode ser substituído pelo modelo da figura (12), equação (3.2).

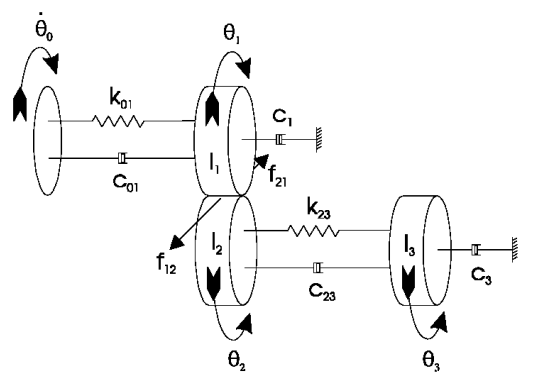

Figura 12 - Modelo Adotado com Entrada Velocidade Constante 
3.3.2.2 Projeto e Implementação da Lei de Movimento com Nonlinear Feedforward

A figura (12) mostra que o modelo dinâmico do sistema é constituído de dois modelos dinâmicos lineares, acoplados entre si pelo contato entre as superfícies do came e do seguidor. Como o perfil do came não é regular, esse contato pode ser visto como uma não linearidade estática. Para se projetar um novo perfil de came que considere para efeito de compensação a dinâmica do sistema como um todo é necessário se obter a equação diferencial não linear que representa esse sistema. Para se obter essa equação diferencial é preciso desacoplar o sistema da figura (12) em dois subsistemas, como mostrado a seguir.

\subsection{Determinação da Equação Diferencial Não Linear}

Para se proceder à compensação dinâmica do sistema por nonlinear feedforward deve-se inicialmente desacoplar as equações entre o subsistema de acionamento, transmissão e came, daquele outro subsistema seguidor constituído pelo seguidor, barra torcional de transmissão e carga. A figura (13) mostra esta situação. Esse desacoplamento se faz com base em considerações sobre o equilíbrio instantâneo de potência. 


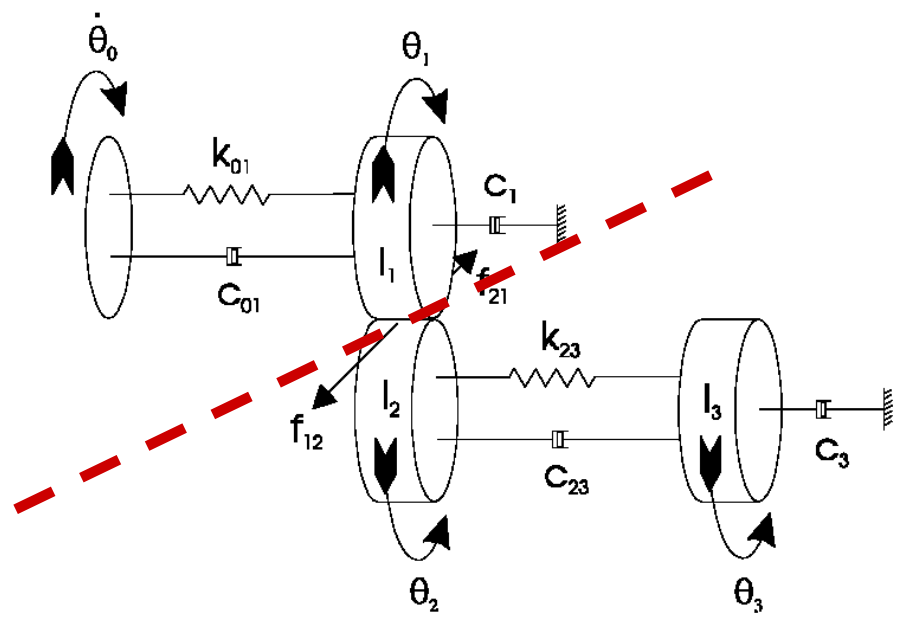

Figura 13 - Desacoplamento dos Subsistemas

Assim, pode-se então desacoplar os dois subsistemas de forma a se proceder à modelagem matemática de cada um deles. O primeiro subsistema pode ser visto isoladamente na figura (14), onde a entrada é a velocidade angular constante no eixo do motor.

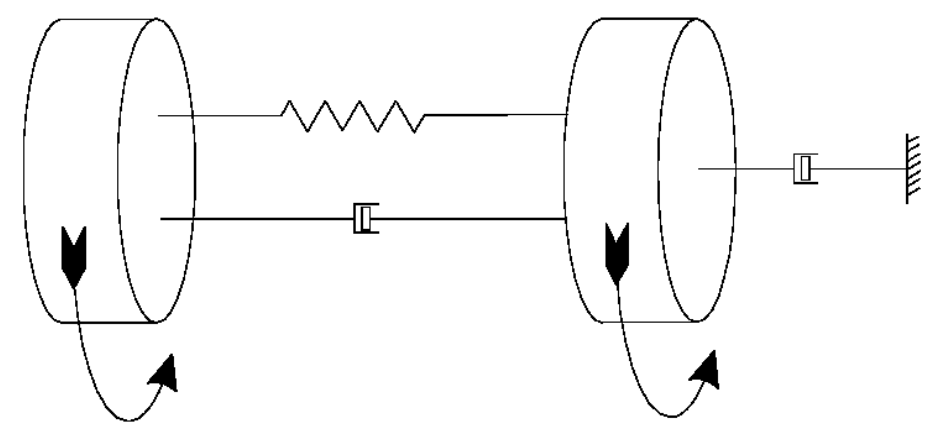

Figura 14 - Subsistema de Acionamento, Transmissão e Came

- Aplicando a $2^{\mathrm{a}}$ Lei de Newton a 11, e desprezando-se 0 atrito estrutural no acoplamento vem: 


$$
-k_{01}\left(\theta_{1}-\theta_{0}\right)-c_{1}\left(\dot{\theta}_{1}\right)-T_{1}=I_{1} \ddot{\theta}_{1}
$$

O segundo subsistema desacoplado pode ser visto na figura (15)

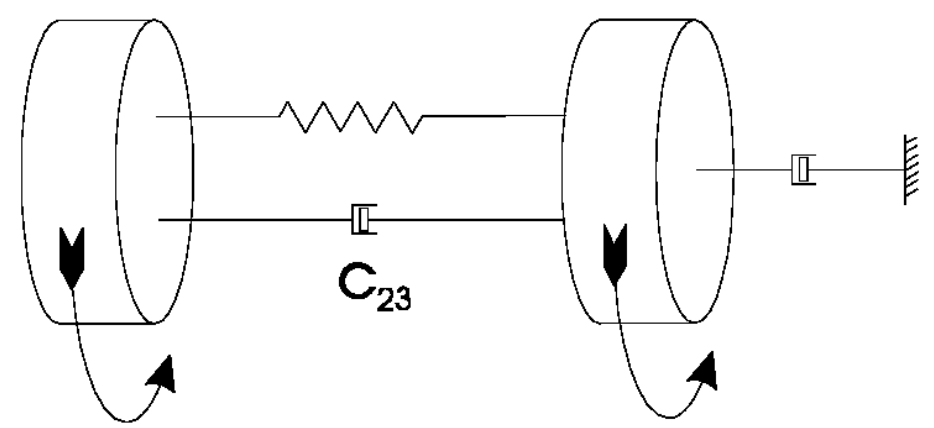

Figura 15 - Subsistema Seguidor

- Aplicando a 2a Lei de Newton a 12 e desprezando-se o atrito estrutural na barra:

$$
-k_{23}\left(\theta_{2}-\theta_{3}\right)+T_{2}=I_{2} \ddot{\theta}_{2}
$$

- Para a inércia /3 tem-se:

$$
-k_{23}\left(\theta_{3}-\theta_{2}\right)-c_{3}\left(\dot{\theta}_{3}\right)=I_{3} \ddot{\theta}_{3}
$$

- Sendo $P$ a potência transmitida entre os subsistemas:

$$
P=T_{1} \cdot \dot{\theta}_{1}=T_{2} \cdot \dot{\theta}_{2}
$$

- Escrevendo T1 em função de T2, vem : 


$$
T_{1}=\frac{T_{2} \cdot \dot{\theta}_{2}}{\dot{\theta}_{1}}
$$

- Substituindo na equação do sistema 1:

$$
I_{1} \ddot{\theta}_{1}+k\left(\theta_{1}-\theta_{0}\right)+c_{1}\left(\dot{\theta}_{1}\right)=\frac{T_{2} \dot{\theta}_{2}}{\dot{\theta}_{1}}
$$

- Onde T2 pode ser obtido da equação do sistema 2 :

$$
T_{2}=I_{2} \ddot{\theta}_{2}+k_{23}\left(\theta_{2}-\theta_{3}\right)
$$

- Pode-se observar que T2 não depende de $\theta 1$.

- Escrevendo na forma de variáveis de estado:

$$
\left[\begin{array}{c}
\ddot{\theta}_{1} \\
\dot{\theta}_{1}
\end{array}\right]=\left[\begin{array}{cc}
-\frac{c_{01}}{l_{1}} & -\frac{k_{01}}{l_{1}} \\
1 & 0
\end{array}\right]\left[\begin{array}{c}
\dot{\theta}_{1} \\
\theta_{1}
\end{array}\right]+\left[\begin{array}{c}
k_{01} \theta_{0}+c_{01} \dot{\theta}_{0}+\frac{T_{2} \dot{\theta}_{2}}{l_{1} \dot{\theta}_{1}} \\
0
\end{array}\right]
$$

- Conforme visto anteriormente:

$$
T_{2}=I_{2} \ddot{\theta}_{2}+k_{23}\left(\theta_{2}-\theta_{3}\right)
$$

- O novo perfil do came é então descrito pela relação:

$$
\theta_{2}(t)=f_{n l f} \boldsymbol{Q}_{1}(t)
$$

- Ou na forma de componentes harmônicas:

$$
\theta_{2}(t)=f_{n l l f} \boldsymbol{\theta}_{1}(t)=h_{0}+\sum \boldsymbol{\sigma}_{i} \cos \left(i \theta_{1}(t)+\varphi_{i}^{-}\right.
$$




\subsection{Interpretação da Equação Diferencial Não Linear}

A equação diferencial obtida no item anterior, ao se fazer o desacoplamento dos dois subsistemas que compõem o sistema completo de came e seguidor, fornece uma relação entre os movimentos do came e do primeiro elemento do seguidor. O movimento que o came geraria no seguidor (elemento 2) e também no elemento final do subsistema seguidor (elemento 3) se não houvesse efeitos dinâmicos nesse subsistema corresponde ao perfil do came nominal, sem compensação alguma. Esse movimento deve então ser imposto ao último elemento do subsistema seguidor (elemento 3) e, através do método de linear feedforwarding, obtém-se o movimento que deve ser realizado pelo elemento 2 . Verifica-se assim que mesmo nos casos em que a compensação por linear feedforwarding apresenta restrições, essa compensação é utilizada no projeto da compensação por nonlinear feedforwarding. Uma vez que o movimento ideal do elemento 2 está assim definido, o sistema completo de came e seguidor se reduz ao primeiro subsistema mais uma lei de movimento, que agora tem que superar o torque variável necessário para produzir o movimento ideal. A equação diferencial não linear obtida no item anterior permite a obtenção do perfil do came compensado não linearmente ao relacionar os movimentos do came e do primeiro elemento do seguidor.

Os coeficientes hi e pi da equação (3.24) são assim obtidos através de um ajuste por mínimos quadrados na função do perfil do came obtida pelo método linear feedforward e a solução $\theta 1$ resultante da equação diferencial (3.18). 
3.3.2.2.3 Implementação da Lei de Movimento com Nonlinear Feedforward

Uma vez gerado o novo perfil de came com a compensação por nonlinear feedforwarding o comportamento do sistema pode ser simulado e também analisado experimentalmente, de forma a se comparar com o comportamento no caso da compensação por linear feedforwarding. Esse novo came, com a compensação não linear, deve ser capaz de superar as deficiências que podem ser apresentadas pelo de compensação linear nos casos mais críticos. Como caso crítico importante deve-se ressaltar aquele da flutuação de velocidade no eixo do came, produzida na compensação linear com um servomotor subdimensionado. Aqui também, mais uma vez, aparece a importância do came compensado apenas linearmente: nos casos em que se constata a não manutenção de velocidade constante, esta mesma flutuação deve ser usada no processo de obtenção do novo perfil de came com compensação não linear.

\subsubsection{Identificação dos Parâmetros do Sistema}

Para se proceder à identificação dos parâmetros do sistema devem-se levar em conta os dois subsistemas em que pode ser desacoplado o sistema completo de came e seguidor. Assim, procede-se à identificação dos parâmetros do sistema de acionamento, constituído do servomotor, 
transmissão e came, e à identificação do subsistema seguidor, constituído do elemento seguidor e da inércia oscilante movida.

Como essa identificação é necessária para se completar e até mesmo ajustar os modelos dinâmicos utilizados no desenvolvimento dos novos perfis de cames com compensação, é conveniente aqui que se descreva um pouco mais as características dos perfis dos cames.

O primeiro came a ser utilizado é o chamado came nominal, aquele cujo perfil produziria no elemento final do subsistema seguidor o movimento desejado, desconsiderados os efeitos dinâmicos. Os cames utilizados no banco de ensaio foram do tipo "master-slave", de forma a sempre garantir o contato entre as superfícies do came e do seguidor. A figura (16) mostra de forma esquemática o conjunto came-seguidor, onde se observa que o movimento do corpo 2 é função do movimento e do perfil do corpo 1, o came, além das curvas para o seguidor (elemento 2) e para a carga oscilante movida (elemento 3).

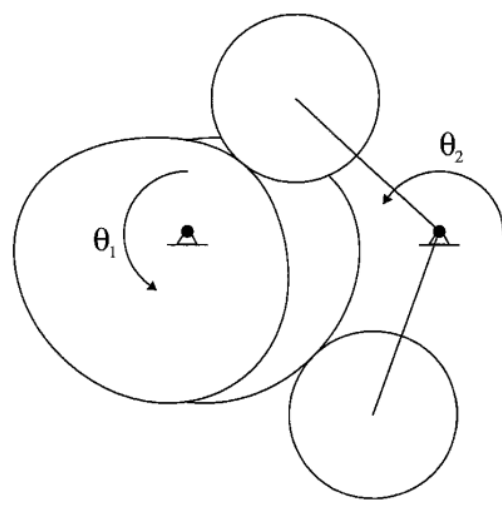

motion law : $\theta_{2}=f\left(\theta_{1}\right)$

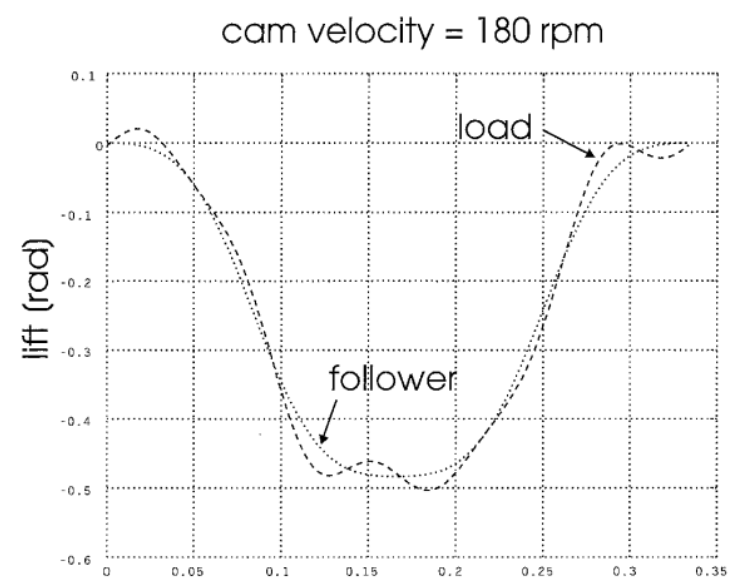

time (s)

Figura 16 - Detalhe do Came e dos Movimentos dos Corpos 3 e 4 
Os movimentos do seguidor (pontilhado) $\Theta_{2}$ e da carga (tracejado) $\Theta_{3}$, mostrados na figura (16), apresentam entre si uma diferença que explicita o efeito da dinâmica do sistema na transmissão do movimento.

O perfil do came original é descrito pela seguinte tabela:

\begin{tabular}{|c|c|c|}
\hline Componente & Amplitude [m] & Fase [rd] \\
\hline 0 & 0.24170000000000 & 0.0 \\
\hline 1 & 0.26670000000000 & 0.0 \\
\hline 2 & 0 & 0.0 \\
\hline 3 & -0.02540000000000 & 0.0 \\
\hline 4 & 0 & 0.0 \\
\hline 5 & 0.00042570000000 & 0.0 \\
\hline 6 & 0 & 0.0 \\
\hline 7 & -0.00004702000000 & 0.0 \\
\hline 8 & 0 & 0.0 \\
\hline 9 & 0.00001073000000 & 0.0 \\
\hline
\end{tabular}

Ao se projetar um novo perfil de came, seja ele aquele com compensação linear ou o com compensação não linear, uma nova tabela como essa é gerada na forma das equações (3.23) e (3.24) com os valores de amplitude e ângulo de fase para cada componente harmônico do novo perfil. Um dado prático 
interessante é que os três perfis de cames utilizados, o nominal, o compensado linearmente, e aquele com compensação não linear, quando colocados lado a lado, apresentam entre si diferenças visualmente desprezíveis.

A figura (17) mostra graficamente o perfil do came nominal projetado originalmente para o sistema em estudo com base em série harmônica limitada de Fourier.

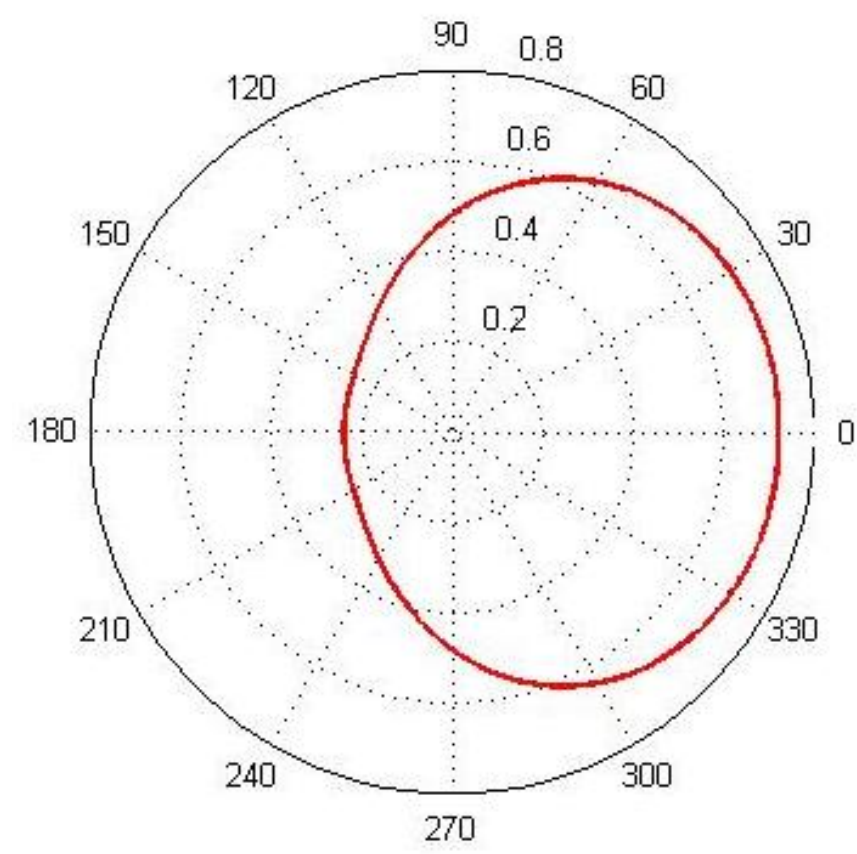

Figura 17 - Perfil do Came Nominal

Para a identificação dos parâmetros foram instalados no banco de ensaios um RVDT, para medir a posição angular do último elemento do subsistema seguidor, $\Theta_{3}$, extensômetros para medir o torque na barra de ligação entre 0 seguidor e a carga, e um tacômetro para medir a velocidade angular no eixo do came, $\Theta_{1}$. 
Com os elementos de medição instalados foram então medidas as seguintes grandezas:

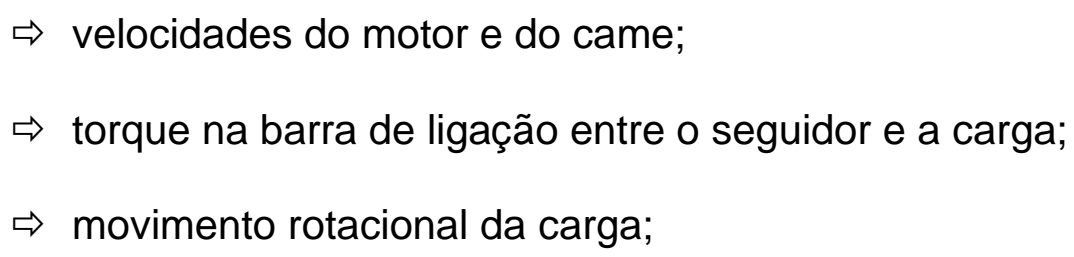

O procedimento utilizado para a identificação dos parâmetros do subsistema seguidor está mostrado esquematicamente na figura (18).

Identification of the Follower System

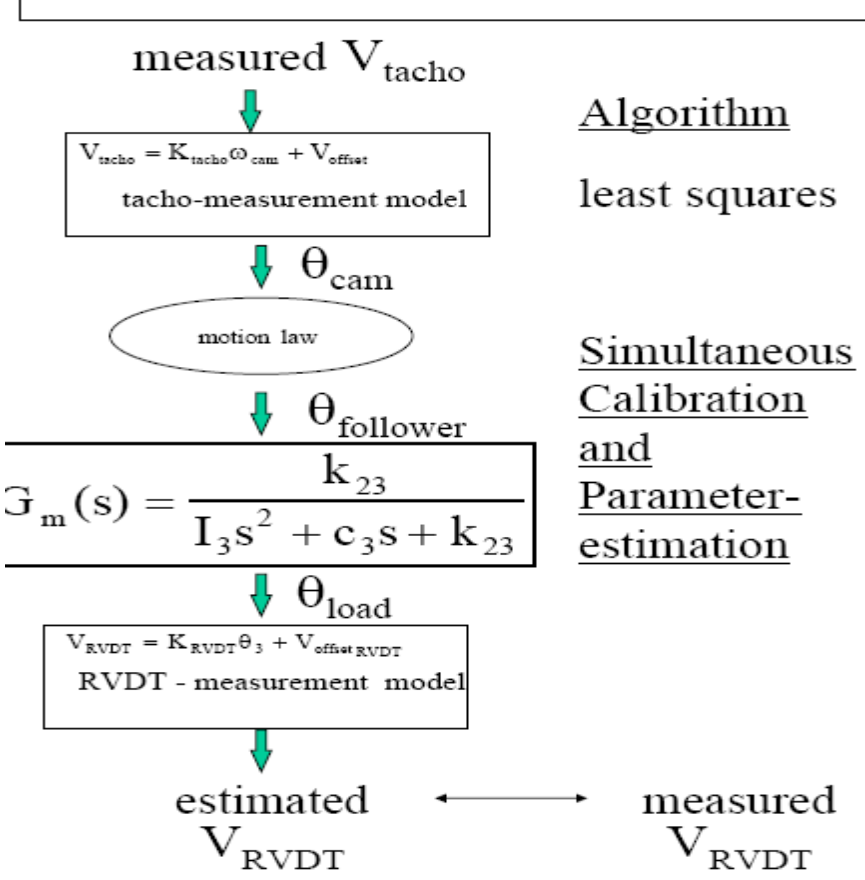

Figura 18 - Diagrama Esquemático do Procedimento para Identificação dos Parâmetros do Subsistema Seguidor 
Os resultados da identificação desses parâmetros do subsistema seguidor são mostrados na figura (19), e esses resultados foram então utilizados para se projetar o novo perfil do came, com a aplicação da compensação por linear feedforward a partir do came nominal.

\section{Identification Results}

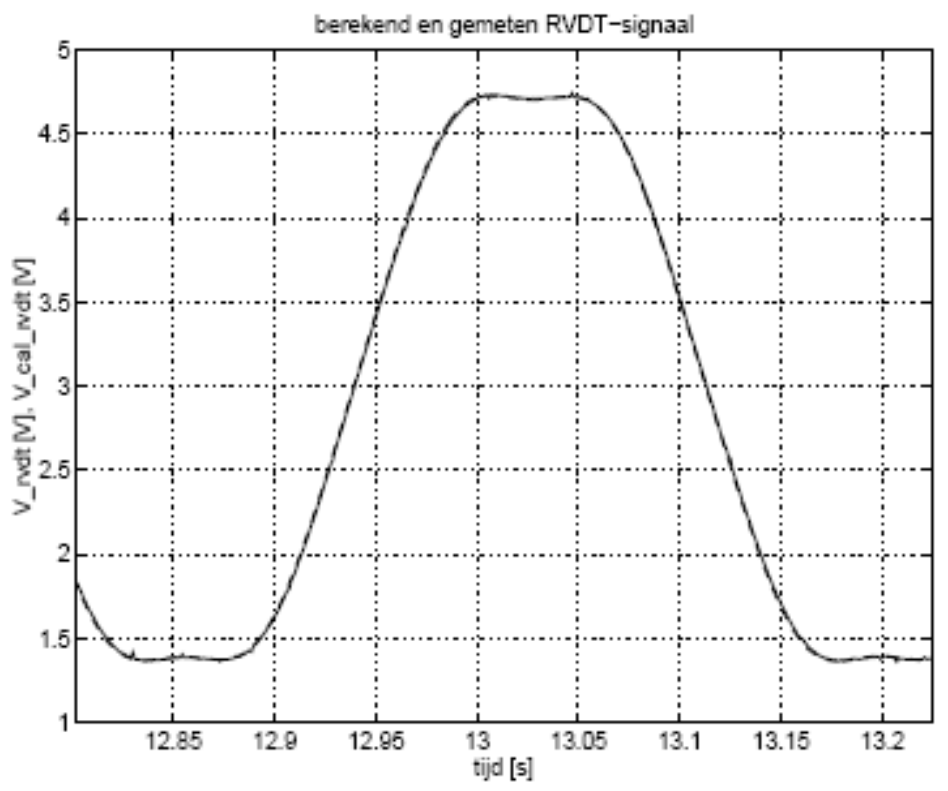

$$
\begin{aligned}
& \mathrm{k}_{23}=3050.14 \mathrm{Nm} / \mathrm{rad} \\
& \mathrm{c}_{3}=0.2622 \mathrm{Nms} / \mathrm{rad} \\
& \mathrm{I}_{3}=0.3550 \mathrm{kgm}^{2}
\end{aligned}
$$

design of the feedforward motion law

Figura 19 - Resultados da Identificação dos Parâmetros do Subsistema Seguidor 
A partir desta identificação foram obtidos os resultados referentes aos parâmetros do subsistema seguidor, a saber, a rigidez torcional da barra que liga o seguidor à carga, o fator de amortecimento no eixo da carga, e a inércia da carga.

Foi também realizado um ensaio de identificação com um martelo de impacto e os resultados estão mostrados na figura (20).

\section{Hammer excitation}
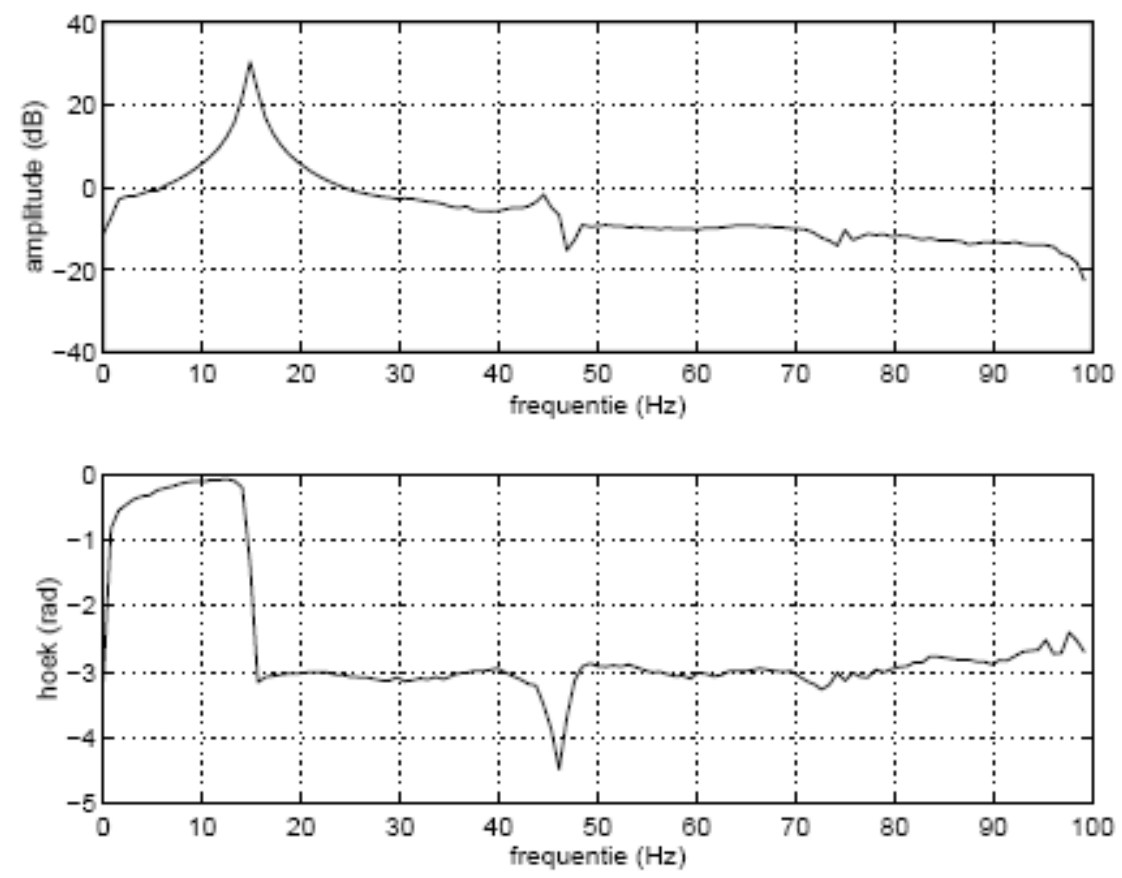

$$
\begin{gathered}
\mathrm{f}_{0}=14.8 \mathrm{~Hz} \\
\zeta \approx 1 \%
\end{gathered}
$$

Figura 20 - Resultados da Identificação de Parâmetros do Subsistema Seguidor 
Para a identificação dos parâmetros do subsistema de acionamento foi adotado inicialmente o modelo mostrado esquematicamente na figura (21).

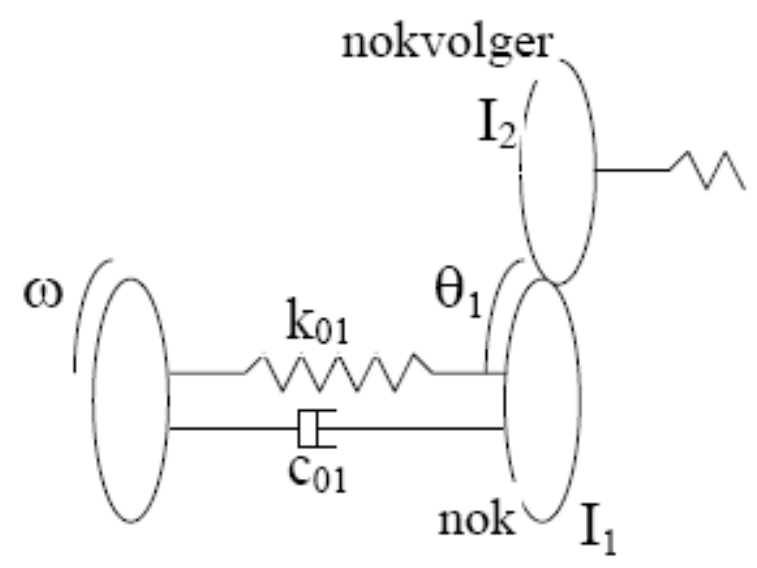

Figura 21 - Modelo Esquemático do Subsistema de Acionamento para Identificação de Parâmetros

O subsistema de acionamento, constituído pelo servomotor com controle PI de velocidade e entrada torque constante, a transmissão com alta rigidez por correia dentada, e o came, foram substituídos na modelagem pelo modelo equivalente entre eixo do motor com velocidade angular constante, flexibilidade na transmissão, e o came. Este último é o modelo esquemático ilustrado na figura (21), onde se observa ainda o seguidor em contato com o came. Os principais problemas encontrados inicialmente na identificação dos parâmetros desse subsistema foram a influência do subsistema seguidor no came, e a saturação do motor em certas partes do ciclo de movimento. A figura (22) mostra o resultado de medição da corrente do motor com a saturação 
juntamente com o registro do movimento do subsistema seguidor, de forma a se verificar a correspondência entre essa saturação e a respectiva parte do ciclo de movimento.

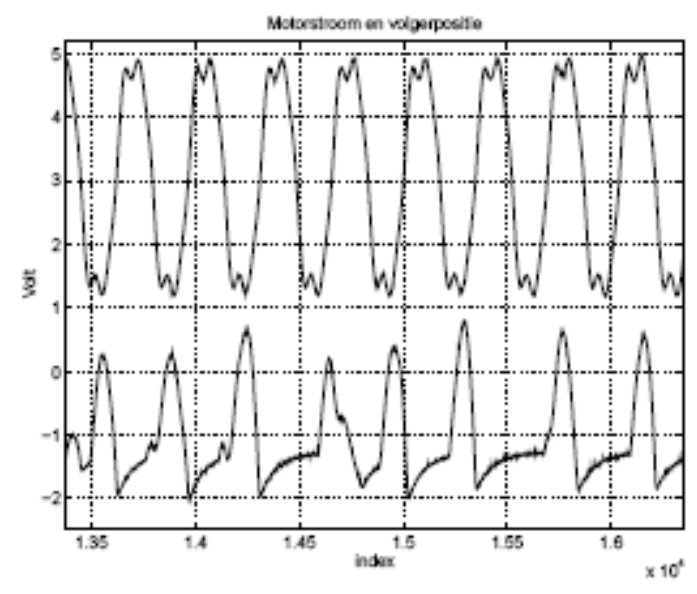

Figura 22 - Registro da Saturação do Motor e o Correspondente Ciclo de Movimento do Subsistema Seguidor

Como resultado do efeito dessa saturação verificou-se prejudicado o modelo inicial do subsistema de acionamento. A saturação ocorria devido à alta demanda de energia durante a ressonância do subsistema seguidor. Como resultado deste fato, pode-se concluir que o came ótimo em termos de comportamento, que viria a ser aquele com compensação por nonlinear feedforward, deveria evitar a ressonância.

A partir dessa constatação da influência do subsistema seguidor no came e a saturação de corrente do motor, buscou-se levar em consideração esses fatos na identificação dos parâmetros do subsistema de acionamento e o procedimento adotado está mostrado de forma esquemática na figura (23). 


\section{Identification of the cam driving system}

- Influence of the follower-train $=$ instant. conservation of the energy (power) $\quad T_{1} \dot{\theta}_{1}=T_{2} \dot{\theta}_{2}$

- $\underline{\text { Model comparison }}=$

$$
\frac{\dot{\theta}_{1}-\dot{\theta}_{0}}{T_{1}}=A(s)=\frac{s}{l_{1} s^{2}+c_{01} s+k_{01}}
$$

- Outline :

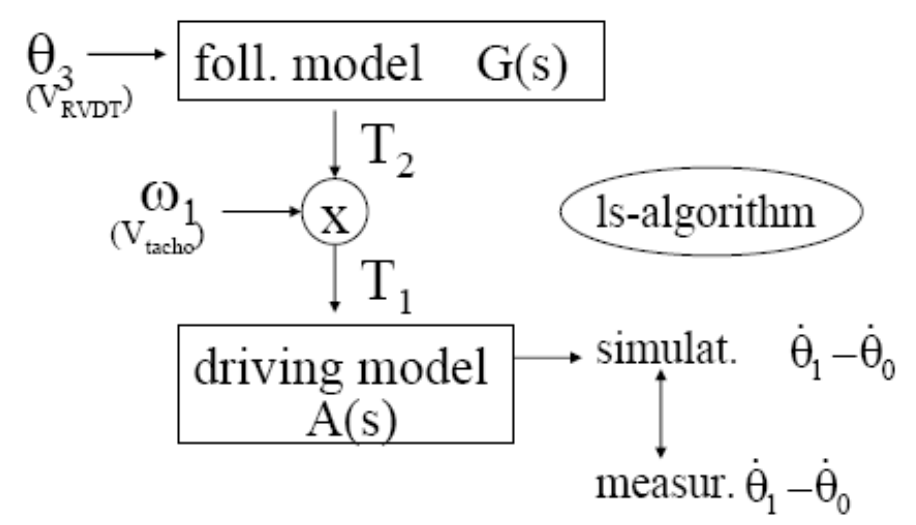

Figura 23 - Diagrama Esquemático do Procedimento para Identificação dos Parâmetros do Subsistema de Acionamento

A figura (24) mostra os resultados da identificação dos parâmetros do subsistema de acionamento e a figura (25) mostra os resultados da análise de sensibilidade com os parâmetros de rigidez, inércia e amortecimento do subsistema de acionamento. 


\section{Identification results}
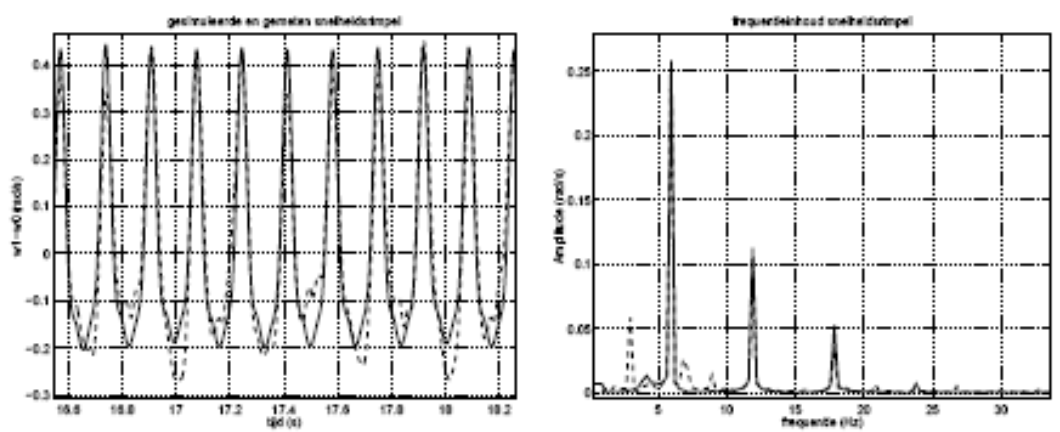

$$
\begin{aligned}
& \mathrm{k} 01=1000 \mathrm{Nm} / \mathrm{rad} \\
& \mathrm{c} 01=4.83 \mathrm{Nms} / \mathrm{rad} \\
& \mathrm{I} 1=1.5 \mathrm{kgm}^{2}
\end{aligned}
$$

Figura 24 - Resultados da Identificação de Parâmetros do Subsistema de Acionamento 


\section{Sensitivity analysis of the cam driving system model}

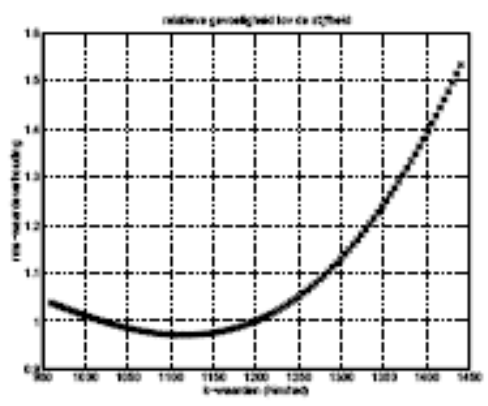

stiffness

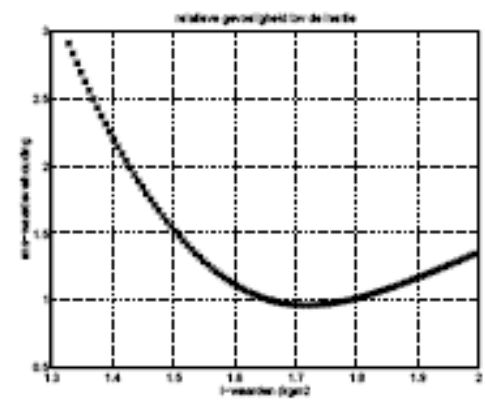

inertia

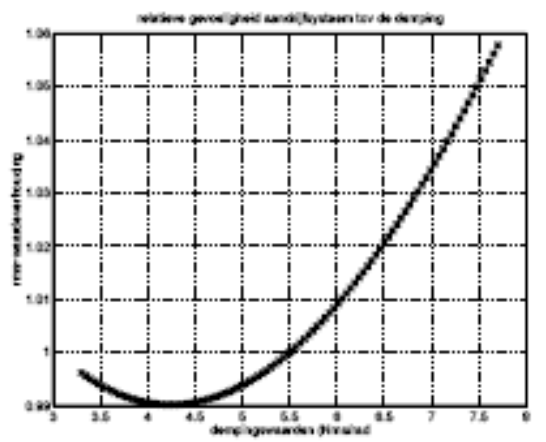

damping

Driving system + follower system identification:

- complete model

Figura 25 - Análise de Sensibilidade de Parâmetros do Subsistema de Acionamento

A partir da identificação dos parâmetros do subsistema seguidor e também dos parâmetros do subsistema de acionamento, resulta determinado o modelo 
dinâmico completo do sistema, que então é utilizado para a obtenção dos novos perfis de cames com compensação.

A figura (26) mostra os dados utilizados para a validação do modelo completo. São utilizados três séries de dados obtidos através de medições da flutuação da velocidade no eixo do came e também da posição angular da carga. Duas destas séries são utilizadas para ajustar os parâmetros do modelo dinâmico que posteriormente é comparado com a outra série de dados experimentais.

\section{Complete model validation}

velocity ripple

Measurement I:
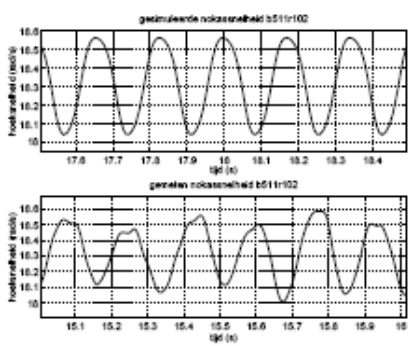

Measurement II :
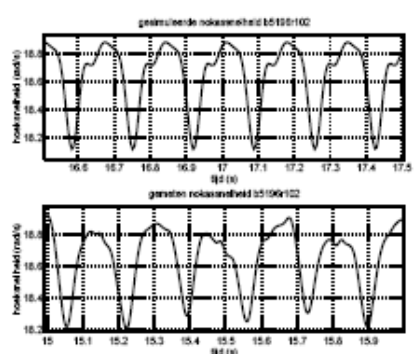

follower motion
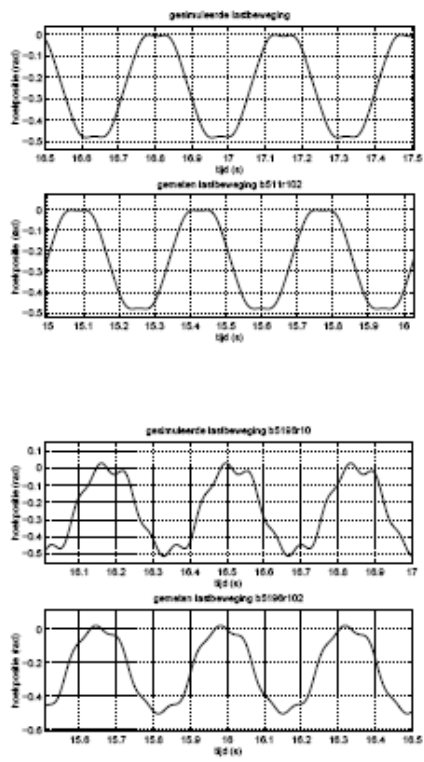

Figura 25 - Dados Experimentais e de Simulação da Flutuação na Velocidade do Came e da Posição Angular da Carga 


\section{Resultados e Análises}

\subsection{Introdução}

Foram examinados três tipos de compensação. A primeira delas, apenas a compensação da dinâmica do subsistema seguidor (linear), aplicada a um modelo reduzido onde era imposta velocidade constante ao eixo do came, desprezando-se assim toda a dinâmica do subsistema de acionamento do came e mesmo a não linearidade estática presente no contato entre o came e o seguidor. Esta primeira compensação, de forma isolada, não se apresenta realista em termos de aplicação prática pois a hipótese de velocidade constante no eixo do came, dadas as restrições do banco de ensaios, não era realista. Ela serve muito bem, entretanto, para ilustrar a idéia básica do método proposto de se aplicar técnicas de controle como feedforward ao projeto de máquinas. A segunda compensação tratou também apenas da compensação da dinâmica do subsistema seguidor (linear), porém aplicando-se o resultado do came correspondente ao sistema completo não linear. A terceira e última compensação tratou da dinâmica do sistema completo não linear, levando em conta estas não linearidades no projeto desse novo perfil de came. 


\subsection{Compensação Linear (Linear Feedforward)}

Um modelo linear permite a obtenção do modelo inverso exato para se obter um came compensado por feedforward a partir do came nominal (original). Como em um sistema não linear não existe um modelo dinâmico inverso, neste caso de compensação por linear feedforwarding o que se fez foi aplicar a dinâmica inversa apenas do modelo do subsistema seguidor (linear) ao came nominal, obtendo-se assim um novo came compensado que foi então utilizado no sistema não linear completo. Os resultados da simulação do sistema completo com esse novo came mostram uma acentuada melhora no desempenho em relação aos resultados obtidos com o came nominal, nos casos em que se mantém constante a velocidade no eixo do came. Os experimentos levados a efeito com esse novo came, entretanto, apresentam resultados diferentes. Isto é devido ao fato de se utilizar um servomotor propositalmente subdimensionado, que não tem potência suficiente para manter a velocidade constante nos períodos do ciclo de movimento onde é maior a demanda. O modelo dinâmico ajustado para essa situação mostra também resultados similares. Para se superar essa situação, em que a velocidade não se mantem constante, algumas possíveis saídas são: aumentar a potência do servomotor (aumenta custos), aumentar o volante auxiliar no eixo do came cuja função é ajudar a manter estável a velocidade, ou aplicar técnicas de compensação de torque (descrito anteriormente). Também com esta finalidade, durante a execução deste trabalho, foi desenvolvido um estudo com um mecanismo inercial adaptativo (ballerina flywheel) para regularização 
de velocidade por compensação de torque. Parte deste estudo é apresentada em um artigo anexo a este trabalho.

A figura (26) mostra alguns resultados experimentais e de simulações com os cames nominal e o com compensação por linear feedforwarding.

\section{SIMULATIONS AND EXPERIMENTAL VERIFICATION}

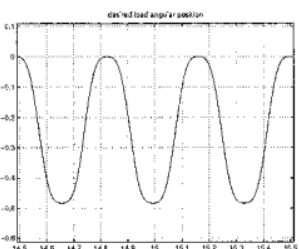

(a)

: (a) desired load angular position

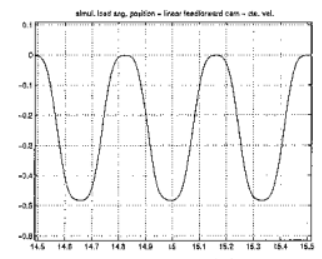

(a)

): (a) simulated load angular position - linear feedforward cam - const.vel.

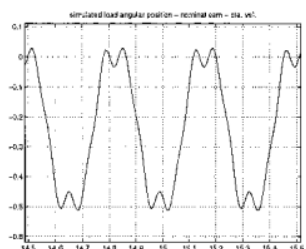

(b)

(b) simulated load angular position - nominal cam - const. vel.

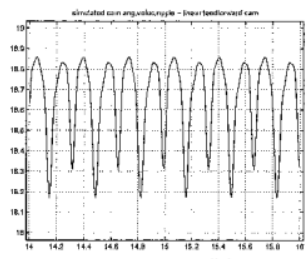

(b)

(b) simulated cam angular velocity ripple - linear feedforward cam

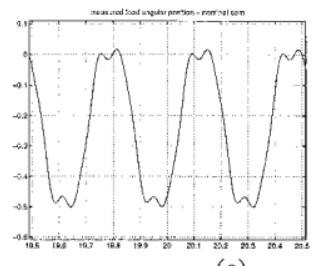

(c)

(c) measured load angular position - nominal cam

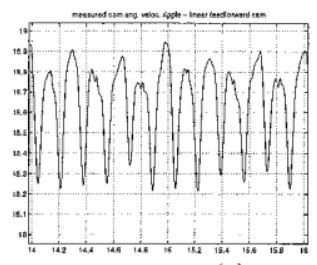

(c)

(c) measured cam angular velocity ripple - linear feedforward cam

Figura 26 - Resultados de Simulações e de Experimentos com os Cames Nominal e com Compensação Linear

Na figura (26), a figura (a) superior mostra o perfil do came nominal, e que também é o movimento desejado para a carga. As figuras (b) e (c) superiores, respectivamente simulação e medida da posição da carga com o came nominal, mostram o efeito da dinâmica do seguidor no resultado final. A figura (a) inferior mostra o movimento da carga com o came compensado linearmente porém com velocidade constante no eixo do came. Já as figuras (b) e (c) 
inferiores, respectivamente simulação e medida, mostram os resultados da flutuação de velocidade no eixo do came quando não se impõe a velocidade constante ao eixo do came. O movimento da carga nesse caso, mesmo com o came compensado linearmente, e devido aos fatores explicados anteriormente, não são então aceitáveis. A figura (27) mostra esses resultados do movimento da carga, tanto na velocidade nominal de operação quanto $10 \%$ abaixo e $10 \%$ acima dessa rotação.

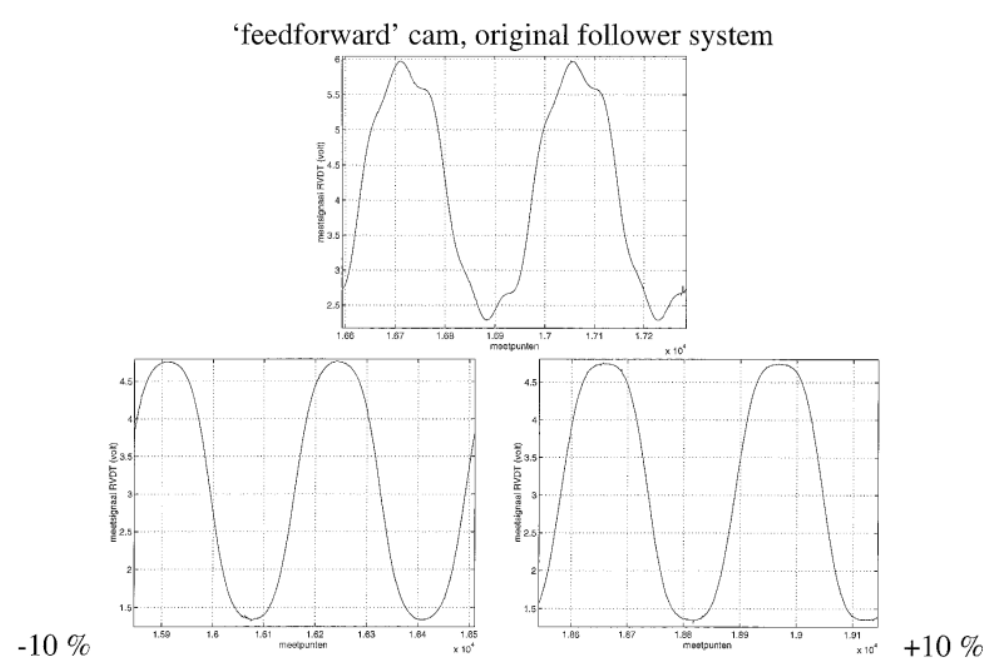

Figura 27 - Resultados Experimentais com o Came Compensado por linear feedforwarding na Rotação Nominal, e 10\% Abaixo e Acima dessa Rotação

Como os resultados com o came compensado linearmente não se mostraram satisfatórios dentro das condições de trabalho propostas, partiu-se então para o projeto do novo came, com a compensação das não linearidades do sistema completo. Para a obtenção desse novo came, com compensação por nonlinear feedforwarding, é fundamental a utilização dos registros de dados experimentais da flutuação da velocidade no eixo do came do caso linear. 


\subsection{Compensação Não Linear (Nonlinear Feedforward)}

Conforme estabelecido anteriormente, a flutuação de velocidade no eixo came em torno do valor nominal deve ser considerada na formulação do perfil do came com compensação por nonlinear feedforwarding. Assim sendo, é conveniente proceder a uma análise dos dados experimentais obtidos sobre essa flutuação de velocidade quando da utilização no sistema do came compensado apenas por linear feedforwarding. A figura (28) mostra o registro dessa flutuação de velocidade em torno do valor nominal, e também uma análise do conteúdo de freqüência desse sinal.
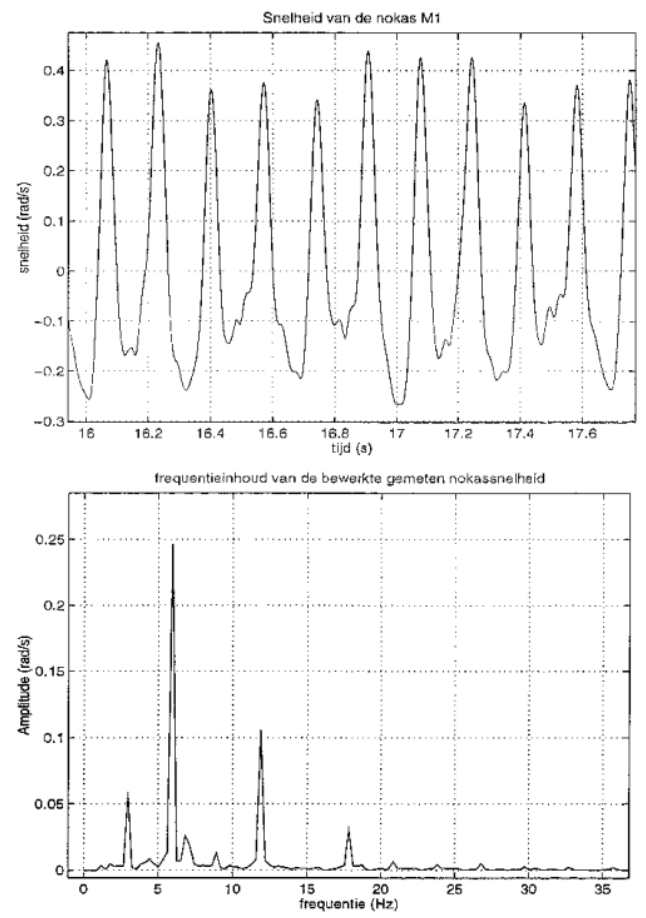

Figura 28 - Dados Experimentais sobre Flutuação da Velocidade no Eixo do Came e Conteúdo de Freqüência desse Sinal 
A partir dos dados mostrados na figura (28) foi feita uma análise desse sinal de forma a se conhecer a influência de cada freqüência no desempenho do sistema.

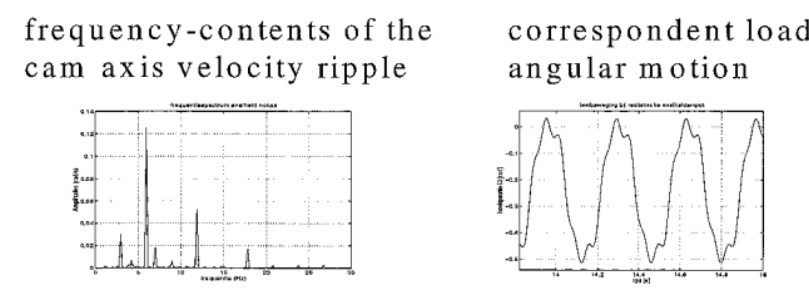

$11-13 \mathrm{~Hz}$ exclusion

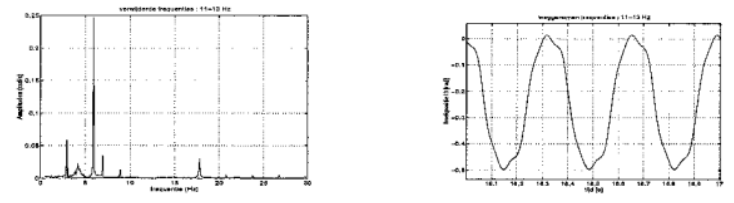

$5-6.5 \mathrm{~Hz}$ exclusion

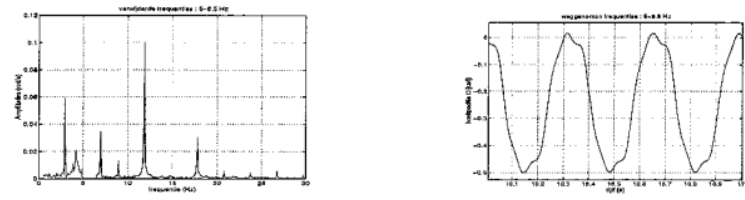

5-6.5 and $11-13 \mathrm{~Hz}$ exclusion
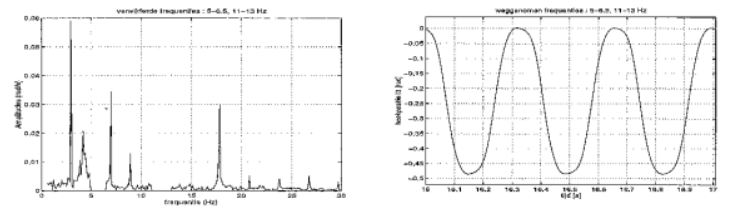

5-6.5 and 11-13 and 18-20 $\mathrm{Hz}$ exclusion
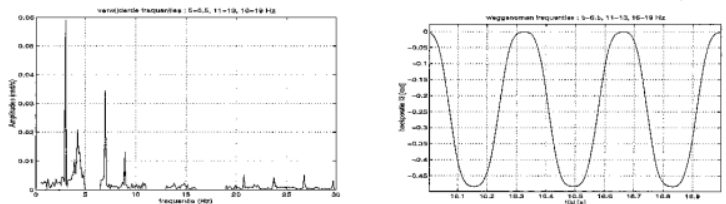

Figura 29 - Análise do Conteúdo de Freqüência nos Dados Experimentais sobre Flutuação da Velocidade no Eixo do Came

De fato, analisando-se os dados da figura (29), verifica-se que o movimento da carga fica distinto daquele desejado devido à flutuação de velocidade no eixo do came e ainda pode-se notar que as freqüências de $6 \mathrm{~Hz}$ e $12 \mathrm{~Hz}$ têm a maior influência nesse comportamento. 
Após a análise e verificação da influência da flutuação da velocidade no desempenho do sistema, utilizou-se desses dados experimentais e também dos dados obtidos ao se desenvolver o came com compensação linear para, conforme descrito no capítulo 3, projetar o novo came com compensação por nonlinear feedforwarding. Os resultados das simulações com esse novo came no modelo completo do sistema desenvolvido anteriormente com base nos dados experimentais do sistema completo são mostrados na figura (30).

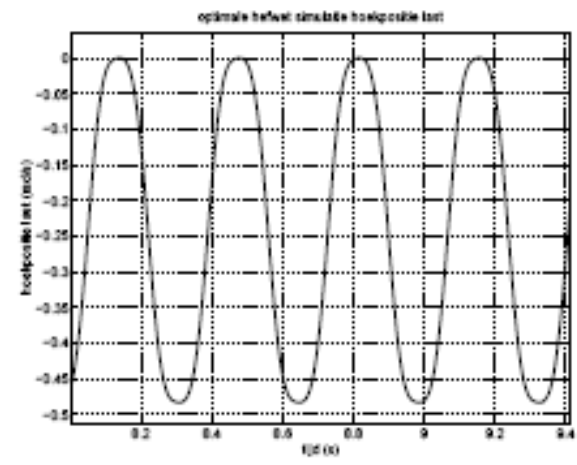

nonlinear feedforward motion law

Figura 30 - Movimento da Carga no Sistema com Compensação por Nonlinear Feedforwarding

Verifica-se que o movimento da carga com este último came com compensação não linear é bastante próximo daquele desejado, ou seja, do movimento "inscrito" originalmente no came nominal, conforme mostrado na figura (26a). As figuras (31) e (32) mostram, respectivamente, os resultados da análise de robustez deste último came em relação a desvios na velocidade angular no eixo do came e em relação a desvios na freqüência natural do subsistema seguidor. 


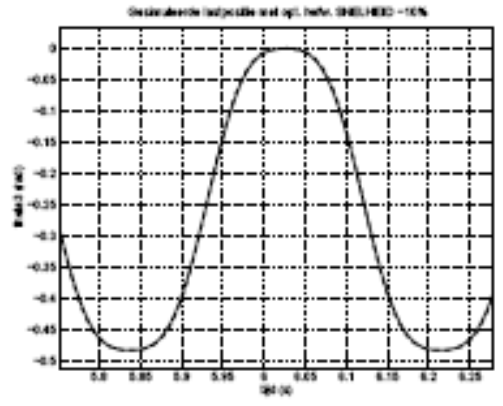

$\omega_{\text {nom }}-10 \%$

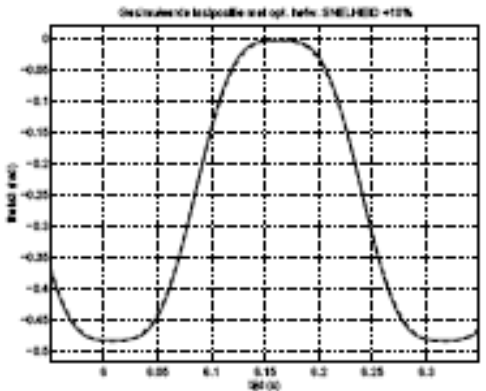

$\omega_{\text {nom }}+10 \%$

Figura 31 - Robustez do Came com Compensação por Nonlinear

Feedforwarding em Relação à Velocidade Angular no Eixo do Came

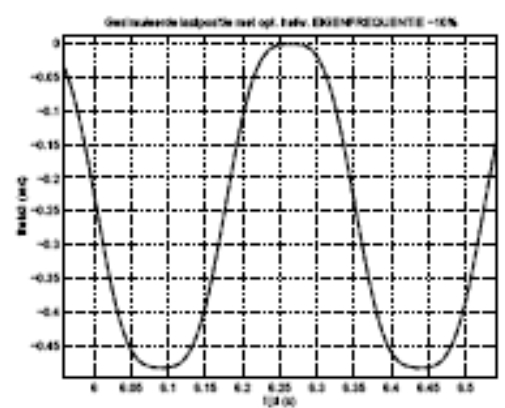

$\omega_{\mathrm{n}}-10 \%$

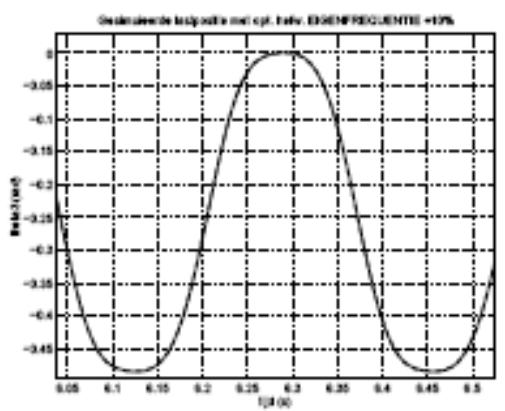

$\omega_{\mathrm{n}}+10 \%$

Figura 32 - Robustez do Came com Compensação por Nonlinear Feedforwarding em Relação à Freqüência Natural do Subsistema Seguidor

Os resultados da análise de robustez mostrada nas figuras (31) e (32), respectivamente em relação à velocidade nominal do eixo do came e em 
relação à freqüência natural do subsistema seguidor, contribuem para atestar o bom desempenho desse último came sintetizado.

\subsection{Conclusões}

De acordo com os resultados obtidos com o processo global de aplicação da técnica de compensação por feedforward pode-se considerar válida essa aplicação. Os resultados finais obtidos com o came compensado linearmente não se mostraram adequados às condições restritivas impostas quando do delineamento do trabalho, mas se mostraram fundamentais durante o processo de obtenção do perfil do came com compensação não linear, e este sim apresentou bons resultados. 


\section{Conclusões Gerais e Trabalhos Futuros}

Como conclusão geral sobre o trabalho desenvolvido pode-se afirmar que o procedimento geral proposto para se desenvolver novos cames de forma a se compensar os efeitos dinâmicos do sistema mostrou um bom potencial para aplicações no projeto desses mecanismos.

O came com compensação apenas linear apresentou bons resultados apenas nas condições restritas onde se garantia uma pequena variação de velocidade angular no eixo do came. Já com a possibilidade de flutuações maiores de velocidade devido à restrições impostas por projeto, por exemplo, o uso de servomotores de potência reduzida por restrições de custo, esse came apresenta resultados que não são tão bons. Quanto maior a flutuação de velocidade no eixo do came, pior é o desempenho do sistema com essa compensação apenas parcial (linear). As restrições que se evidenciaram no emprego deste came, entretanto, deram ensejo ao desenvolvimento de um método para se auxiliar na manutenção da velocidade angular no eixo do came através de um mecanismo que trabalha realizando uma compensação parcial de torque.

O último came projetado e construido, aquele com compensação não linear onde se levou em consideração a dinâmica do sistema não linear completo, apresentou em contrapartida bons resultados, ao menos no caso estudado 
onde o sistema como um todo não apresentava grande complexidade. Esse came, além de apresentar um bom comportamento no que diz respeito a impor à carga o movimento desejado, apresentou também uma boa robustez em relação a desvios na velocidade angular nominal do eixo do came e também em relação a desvios na freqüência natural do subsistema seguidor.

Outra conclusão interessante a respeito do bom funcionamento do sistema com a compensação por nonlinear feedforwarding é que ela é, de certo modo, uma otimização do perfil do came em relação ao movimento desejado do elemento final do subsistema seguidor, considerados aí os efeitos dinâmicos das diversas partes que compõem o sistema completo de came e seguidor.

Em relação a trabalhos futuros, pretende-se extender o método para a aplicação em casos mais complexos, por exemplo, um sistema em que o subsistema seguidor tenha dois graus de liberdade. Também, por se tratar o sistema completo da conjugação de duas partes lineares conectadas por uma não linearidade estática, o contato came-seguidor, deve-se buscar desenvolver modelos mais completos e complexos de forma a se aperfeiçoar os resultados. 


\section{Bibliografia}

1. Alberts, T. E. and H. R. Pota (1997). Broadband Dynamic Modification Using Feedforward Control. Transactions of the ASME, Journal of Dynamic Systems, Measurement, and Control 119(4), 700-706.

2. Alizade, R. I., J. Duffy, and E. T. Hajiyev (1983). Mathematical Models for Analysis and Synthesis of Spatial Mechanisms-I: Four-Link Spatial Mechanisms. Mechanism and Machine Theory 18(5), 301-307.

3. Alizade, R. I., J. Duffy, and A. A. Azizov (1983). Mathematical Models for Analysis and Synthesis of Spatial Mechanisms-II: Five-Link Spatial Mechanisms. Mechanism and Machine Theory 18(5), 309-315.

4. Alizade, R. I., J. Duffy, and E. T. Hajiyev (1983). Mathematical Models for Analysis and Synthesis of Spatial Mechanisms-III: Six-Link Spatial Mechanisms. Mechanism and Machine Theory 18(5), 317-322.

5. Alizade, R. I., J. Duffy, and E. T. Hajiyev (1983). Mathematical Models for Analysis and Synthesis of Spatial Mechanisms-IV: Seven-Link Spatial Mechanisms. Mechanism and Machine Theory 18(5), 323-328.

6. Alizade, R. I., L. G. Novruzbekov, and G. N. Sandor (1975). Optimization of Four-Bar Function Generating Mechanisms Using Penalty Functions with Inequality and Equality Constraints. Mechanism and Machine Theory 10(4), 327-336.

7. Alizade, R. I., A. V. M. Rao, and G. N. Sandor (1975). Optimum Synthesis of Four-Bar and Offset Slider-Crank Planar and Spatial Mechanisms Using the 
Penalty Function Approach With Inequality and Equality Constraints. Transactions of the ASME, Journal of Engineering for Industry 97(3), 785790.

8. Altus, S. S., L. M. Kroo, and P. J. Gage (1996). A Genetic Algorithm for Scheduling and Decomposition of Multidisciplinary Design Problems. Transactions of the ASME, Journal of Mechanical Design 118(4), 486-489.

9. Amman, S. et alli. (19...). Une ouverture rapide de la foule-à quel prix?. 3p..[212]

10.Angeles, J., S. K. Saha, M. González-Palacios, and C. S. López-Cajún (1994). The Design Optimization of Cam Mechanisms With Oscillating FlatFace Followers Under Curvature Constraints. Transactions of the ASME, Journal of Mechanical Design 116(1), 311-314.

11.Angeles, J., S. K. Saha, and C. S. López-Cajún (1994). The Design of Cam Mechanisms With Translating Flat-Face Followers Under Curvature Constraints. Transactions of the ASME, Journal of Mechanical Design 116(1), 306-310.

12.Angst, W. (1983). Entwurf von Kurvenscheiben mit Beschleunigungs-und Geschwindigkeitsblocken. Technica, 11, 857-862, 876.[97-_-c]

13.Andrews, S. W. C. (19..). The Development of 2 and 3 Dimensional Cam Measurement Systems. Precision Grinding Ltd., 11p..[74]

14.Antuma, H. J. (1967). Nokkrommen; mogelijkheden voor optimale keuze; harmonische parabool. Verktuig- en Scheepsbouw, 19, 197-208.[152]

15.Ardayfio, D. D. (1980). Kinetothermoelastodynamic (KITED) Synthesis of Cam Profiles. Mechanism and Machine Theory 15(1), 1-4. 
16.Ardayfio, D. (1978). Stability Analysis of an Acceleration Governor Subjected to a Harmonic Speed Disturbance. Mechanism and Machine Theory 13(2), 119-123.

17.Artobolivskii, A. A. (1975). General Problems in the Theory of Machines and Mechanisms. Mechanism and Machine Theory 10(2/3), 125-130.

18.Asada, H., Z.-D. Ma, and H. Tokumaru (1990). Inverse Dynamics of Flexible Robot Arms: Modeling and Computation for Trajectory Control. Transactions of the ASME, Journal of Dynamic Systems, Measurement, and Control 112(2), 177-185.

19.Backhouse, C. J. (1990). Envelope Theory Applied to Globoidal Cam Surface Geometry. Proc. Instn. Mech. Engrs. 204(C), 409-416.

20.Badlani, M. and A. Midha (1983). Effect of Internal Material Damping on the Dynamics of a Slider-Crank Mechanism. Transactions of the ASME, Journal of Mechanisms, Transmissions, and Automation in Design 105(3), 452-459.

21.Bagci, C. and G. M. Rieser (1984). Optimum Synthesis of Function Generators Involving Derivative Constraints. Mechanism and Machine Theory 19(1), 157-164.

22.Bagci, C. (1984). Geometric Methods for the Synthesis of Spherical Mechanisms for the Generation of Functions, Paths and Rigid-Body Positions Using Conformal Projections. Mechanism and Machine Theory 19(1), 113127.

23.Bagci, C. and S. Kurnool (1997). Exact Response Analysis and Dynamic Design of Cam-Follower Systems Using Laplace Transforms. Transactions of the ASME, Journal of Mechanical Design 119(3), 359-369. 
24.Bagci, C. and S. Kurnool (1994). Exact Response Analysis and Dynamic Design of Cam-Follower Systems Using Laplace Transforms. ASME, Advances in Design Automation, DE-69(1), 613-629.[206]

25.Bagci, C. (1975). Dynamic Motion Analysis of Plane Mechanisms With Coulomb and Viscous Damping via the Joint Force Analysis. Transactions of the ASME, Journal of Engineering for Industry 97(2), 551-560 (+ erratum = 97(3), 1148).

26.Bagepalli, B. S., T. L. Haskins, and I. Imam (1991). Generalized Modeling of Dynamic Cam-follower Pairs in Mechanisms. Transactions of the ASME, Journal of Mechanical Design 113(2), 102-113.

27.Balchanowski, K. J. and A. Gronowicz (1995). Asymmetric Coupler Curves for the Jerk Free Intermittent Motion. In Proceedings of the Ninth World Congress on the Theory of Machines and Mechanisms, Milan, Italy, pp. 718722.

28.Bapat, V. A. and H. V. Kumaraswamy (1979). Effect of Primary Damping on the Tuning Conditions of a Dynamic Vibration Absorber. In Proceedings of the Fifth World Congress on the Theory of Machines and Mechanisms, Montreal, Canada, pp. 329-332.

29.Barton, L. O. (1982). A New Way to Analyze Slider Cranks. Machine Design, July 22, 49-53.[93b-]

30.Barton, L. O. (1979). Painless Analysis of Four-Bar Linkages. Machine Design, July 26, 124-127.[52]

31.Baru, M. K. and J. Ellis (1988). A new approach for the determination of cam follower system parameters. Proc. Instn Mech Engrs 202(C5), 361-368.[150] 
32.Bauer, P. (1982). Methodische Auslegung und Fehlersuche bei Kurvengetrieben. Antriebstechnik, 21(6), 277-281.[96b-]

33.Berger, D. (1977). Ein einfaches Verfahren zur rechnergestutzten Getriebe__konstruktion Feinwerktechnik \& Messtechnik, 85(8), 393-400.[82a-x]

34.Berzak, N. (1982). Optimization of Cam-Follower Systems With Kinematic and Dynamic Constraints. Transactions of the ASME, Journal of Mechanical Design 104(1), 29-33.

35.Berzak, N. and F. Freudenstein (1979). Optimization Criteria in Polydyne CAM Design. In Proceedings of the Fifth World Congress on the Theory of Machines and Mechanisms, Montreal, Canada, pp. 1303-1306.

36.Bialkowicz, B., T. Klimowicz, and M. Swietlik (1979). Changes of the Dynamic Properties of the Real Cam Profile During Its Wear. In Proceedings of the Fifth World Congress on the Theory of Machines and Mechanisms, Montreal, Canada, pp. 984-987.

37.Bijloo, J. A. (1983). De optimale nokfunctie. Bedrijfsmechanisatie-Kern Contactblad, 32(1), March, 12-18.[117]

38.Blejwas, T. E. (1981). The Simulation of Elastic Mechanisms Using Kinematic Constraints and Lagrange Multipliers. Mechanism and Machine Theory 16(4), 441-445.

39.Bögelsack, G., F. J. Gierse, V. Oravský, J. M. Prentis, and A. Rossi (1983). TERMINOLOGY FOR THE THEORY OF MACHINES AND MECHANISMS. Mechanism and Machine Theory 18(6), 379-408.

40.Borovac, B. M. Vokobratovic, and D. Stokic (1983). Analysis of the Influence of Actuator Model Complexity on Manipulator Control Synthesis. Mechanism and Machine Theory 18(2), 113-122. 
41.Brát, V. (1981). IFToMM Reports. Mechanism and Machine Theory 16(3), 263.

42.Braun, E. (1982). NC-Programmiersystem zur Erstellung von Kurvenscheiben, Nocken und Trommelkurven. Metallbearbeitung 76, 82(1), 44-45.[91b-]

43.Brock, R. et alli. (1974). Rationalisierung der Auslegung von Getrieben mit Hilfe von Programmierbaren Tischrechnern. Maschinenbautechnik, 26(8), 1977.[87_-b]

44.Brooke, E. R. (1979). Where the Mechanical Way Still Makes Sense. Machine Design, January 11, 80-83.[35]

45.Brookfield, D. J. (1994). Identification of Coulomb Friction in Robot Drives and other Mechanical Systems through Observation of Third Harmonic Generation. Proc. Instn. Mech. Engrs. 208(C), 329-336.

46.Browning, R. J. (1991). Detroit'91: Overhead cams come out of the closet. Machine Design, January 10, 76-81.[173]

47.Burton, P. (1979). Kinematics and Dynamics of Planar Machinery. New Jersey: Prentice-Hall, Inc. Englewood Cliffs.

48.Bush, G. S., M. O. M. Osman, and S. Sankar (1984). On the Optimal Design of Multi-Speed Gear Trains. Mechanism and Machine Theory 19(2), 183-195. 49.Cai, C. W., H. C. Chan, and Y. K. Cheung (1997). Localized Modes in Periodic Systems With Nonlinear Disorders. Transactions of the ASME, Journal of Applied Mechanics 64(4), 940-945.

50.Carmichael, M. W. J. (1979). Elementary Joystick Mechanisms. In Proceedings of the Fifth World Congress on the Theory of Machines and Mechanisms, Montreal, Canada, pp. 1464-1467. 
51.Cavalcanti, S. G. and W. M. Monsour (1979). Gyroscopic Rollerons for Roll Stabilization of Submersibles. In Proceedings of the Fifth World Congress on the Theory of Machines and Mechanisms, Montreal, Canada, pp. 1506-1509.

52.Chakraborty, J. and S. G. Dhande (1977). Kinematic and Geometry of Planar and Spatial Cam Mechanisms. New York: John Wiley \& Sons.

53.Chakraborty, J. (1975). Synthesis of Mechanical Error in Linkages. Mechanism and Machine Theory 10(2/3), 155-165.

54.Chan, C. and A. P. Pisano (1990). On The Synthesis of Cams With Irregular Followers. Transactions of the ASME, Journal of Mechanical Design 112(1), $36-41$.

55.Chan, C. and A. P. Pisano (1987). Dynamic Model of a Fluctuating RockerArm Ratio Cam System. Transactions of the ASME, Journal of Mechanisms, Transmissions, and Automation in Design 109(3), 356-365.

56.Chapman, C. D. and M. J. Jakiela (1996). Genetic Algorithm-Based Structural Topology Design With Compliance and Topology Simplification Considerations. Transactions of the ASME, Journal of Mechanical Design 118(1), 89-98.

57.Chassapis, C. and G. G. Lowen (1994). The Elastic-Dynamic Modeling of a Press Feed Mechanism. Transactions of the ASME, Journal of Mechanical Design 116(1), 238-247.

58.Chassiakos, A. G., S. F. Masri, A. W. Smyth, and T. K. Caughey (1998). OnLine Identification of Hysteretic Systems. Transactions of the ASME, Journal of Applied Mechanics 65(1), 194-203.

59.Chen, F. Y. (1982). Mechanics and Design of Cam Mechanisms. New York: Pergamon Press. 
60.Chen, F. Y. (1982). Motion Specifications by Other Polynomials (chapter 4 of the book from Chen: Chen, F. Y., 1982, Mechanics and Design of Cam Mechanisms, $2^{\text {nd }}$ ed. New York: Pergamon Press).[160]

61.Chen, F. Y. (1979). A Least-Squares Finite-Difference Method for Kinematic Analysis. In Proceedings of the Fifth World Congress on the Theory of Machines and Mechanisms, Montreal, Canada, pp. 1024-1027.

62.Chen, F. Y. (1977). A Survey of the State of the Art of Cam System Dynamics. Mechanism and Machine Theory, 12, 201-224.[20]

63.Chen, F. Y. and N. Polvanich (1975). Dynamics of High-Speed Cam-Driven Mechanisms - Part 2: Nonlinear Systems Models. Transactions of the ASME, Journal of Engineering for Industry 97(3), 777-784.

64.Chen, F. Y. and N. Polvanich (1975). Dynamics of High-Speed Cam-Driven Mechanisms - Part 1: Linear Systems Models. Transactions of the ASME, Journal of Engineering for Industry 97(3), 769-776.

65.Chen, F. Y. (1973). Kinematic Synthesis of Cam Profiles for Prescribed Acceleration by a Finite Integration Method. Trans. ASME, Journal of Engineering for Industry, May, 519-524.[71]

66.Chen, F. Y. (1973). Analysis and Design of Cam-Driven Mechanisms With Nonlinearities. Transactions of the ASME, Journal of Engineering for Industry 95(3), 685-694.

67.Chen, F. Y. (1972). A Refined Algorithm for Finite-Difference Synthesis of Cam Profiles. Mechanism and Machine Theory 7(4), 453-460.

68.Chen, F. Y. (19..). A Review of the Literature on the Dynamics of Cam Mechanisms., 23-36.[85a-_] 
69.Chew, M. and C. H. Chuang (1995). Minimizing Residual Vibrations in HighSpeed Cam-Follower Systems Over a Range of Speeds. Transactions of the ASME, Journal of Mechanical Design 117(1), 166-172.

70.Chew, M., F. Freudenstein, and R. W. Longman (1983). Application of Optimal Control Theory to the Synthesis of High-Speed Cam-Follower Systems. Part 2: System Optimization. Transactions of the ASME, Journal of Mechanisms, Transmissions, and Automation in Design 105(3), 585-591.

71.Chew, M., F. Freudenstein, and R. W. Longman (1983). Application of Optimal Control Theory to the Synthesis of High-Speed Cam-Follower Systems. Part 1: Optimality Criterion. Transactions of the ASME, Journal of Mechanisms, Transmissions, and Automation in Design 105(3), 576-584.

72.Choi, S.-B. and C.-H. Lee (1997). Force Tracking Control of a Flexible Gripper Driven by Piezoceramic Actuators. Transactions of the ASME, Journal of Dynamic Systems, Measurement, and Control 119(3), 439-446.

73.Choubey, M. and A. C. Rao (1981). Cams and Manufacturing Tolerances. Precision Engineering, 0141, 33-36.[01]

74.Chuenchom, T. and S. Kota (1997). Synthesis of Programmable Mechanisms Using Adjustable Dyads. Transactions of the ASME, Journal of Mechanical Design 119(2), 232-237.

75.Chunhe, H. and L. Shujun (1995). On the Calculating Basic Sizes of Disk Cam with Oscillating Roller Follower in Accordance with Allowable Pressure Angle. In Proceedings of the Ninth World Congress on the Theory of Machines and Mechanisms, Milan, Italy, pp. 732-734. 
76.Cleghorn, W. L., R. G. Fenton, and B. Tabarrok (1981). Finite Element Analysis of High-Speed Flexible Mechanisms. Mechanism and Machine Theory 16(4), 407-424.

77.Cleghorn, W. L., R. G. Fenton, and B. Tabarrok (1981). Optimum Design of High-Speed Flexible Mechanisms. Mechanism and Machine Theory 16(4), 399-406.

78.Craig, J. J. (19..). Introduction to Robotics: Mechanics \& Control, chapter 7: Trajectory Generation..[172]

79.Crossley, F. E. (1988). Recollections From Forty Years of Teaching Mechanisms. Transactions of the ASME, Journal of Mechanisms, Transmissions, and Automation in Design 110(3), 232-242.

80.Crossley, F. R. E., A. Oledzki, and W. Szydlowski (1979). On the Modelling of Impacts of Two Elastic Bodies Having Flat and Cylindrical Surfaces With Application to Cam Mechanisms. In Proceedings of the Fifth World Congress on the Theory of Machines and Mechanisms, Montreal, Canada, pp. 10901092.

81.Cuccio, A. (1991). A Model for a Mechanical Assembly Doing Intermittent Motion. Eighth World Congress on the Theory of Machines and Mechanisms, Prague, Czechoslovakia, 543-546.[166]

82.Cuccio, A and G. Mimmi (1980). Computer Aided Interactive Design of a Mechanism for Intermittent Motion. Italian Machinery and Equipment, 56, 3541.[12]

83.Cuypers, M. H. (1980). Numerical Evaluation of the Torque Capacities of Mechanical Drives, Especially for Continuosly Variable Transmissions. Trans. 
ASME, Century 2 International Power Transmissions \& Gearing Conference, San Francisco, Calif., August 18-21, 14p..[100--c]

84.Dallas, D.B. (1975). Making Trapped Cams by Fine Blanking. Manufacturing Engineering, August.[21]

85.Datseris, P. and W. Palm (1985). Principles on the Development of Mechanical Hands Which Can Manipulate Objects by Means of Active Control. Transactions of the ASME, Journal of Mechanisms, Transmissions, and Automation in Design 107(2), 148-156.

86.Davidson, J. K. (1977). Programmable Calculators take the Drudgery out of LINKAGE ANALYSIS. Machine Design, June 9, 93-97.[102]

87.De Fraine, J. (1995). Anti Vibrations Cam Motion Laws. In Proceedings of the Ninth World Congress on the Theory of Machines and Mechanisms, Milan, Italy, pp. 748-750.

88.De Fraine, J. (1991). Force Analysis in Cams. In Proceedings of the Eighth World Congress on the Theory of Machines and Mechanisms. Prague, Czechoslovakia, pp. 687-690.

89.De Fraine, J. (1983). Utilization de L'Ordinateur pour la Conception et la Fabrication des Cames. BMB Industrial Systems 6, 47-50.[84_-b]

90.De Fraine, J. (1979). Integration of Computer Aided Design and Computer Aided Manufacturing for Cams Driving Mechanisms. Proc. 5th World Cong. on Theory of Machines and Mechanisms, ASME, 1, 122-125.[24]

91.De Fraine, J. (1979). Integration of Computer Aided Design and Computer Aided Manufacturing for CAMS Driving Mechanisms. In Proceedings of the Fifth World Congress on the Theory of Machines and Mechanisms, Montreal, Canada, pp. 122-125. 
92.De Fraine, J. (1978). Computergesteund ontwerp en fabricage van nokken. Metaalbewerking 15, 1-4.[23]

93.De Fraine, J. et B. Pauwels (19..). Le Calcul de Mécanismes de Machines Textiles à l'aide de l'ordinateur. Revue Tijdschrift, 26(4), 1-4.[25]

94.Deep, G. S. and C. S. Chandrasekhar (1979). A Portable Electronic Vehicle Weighing Device. In Proceedings of the Fifth World Congress on the Theory of Machines and Mechanisms, Montreal, Canada, pp. 65-68.

95.Dhande, S. G., B. S. Bhadoria, and J. Chakraborty (1975). A Unified Approach to the Analytical Design of Three-Dimensional Cam Mechanisms. Transactions of the ASME, Journal of Engineering for Industry 97(1), 327333.

96.Deck, J. F. and S. Dubowsky (1994). On the Limitations of Predictions of the Dynamic Response of Machines with Clearance Connections. Transactions of the ASME, Journal of Mechanical Design 116(3), 833-841.

97.(DET) Klein Breteler, A. J. (1988). Discussie-Bijdrage Rond Het Thema Mechatronica. DET Colloquium.[196]

1. (DET) Dijksman, E. A. and A. T. J. M. Smals (1989). The fundamentals about stretchable four-bars, meeting three accuracy positions of the coupler plane. DET - Colloquium te Delft.[184]

2. (DET) Meijaard, J. P. (1989). Het Bepalen van Periodieke Oplossingen van de Niet-Lineaire Dynamica-Vergelijkingen. DET - Colloquium te Delft.[185]

3. (DET) Van Heijningen, G. J. J., and C. M. Kalker-Kalkman (1989). Computer Aided Design of Externally Pressurized Bearings. (DET - Colloquium te Delft ?) $1989(8)(?) \cdot[186]$ 
4. (DET) Sytstra, A. L. (1988). Kinematical Analysis of Transmissions based on the finite element method. A summary of the D.E.T. colloquium d.d.,.[187]

5. (DET) Dijksman, E.A.(?) (1989). Instantaneous four-bar Kinematics based on the Carter-Hall circle. DET - Colloquium, “Leer der Mechanismen”,.[188]

6. (DET) Dijksman, E.A. and H. A. Bulten(?) (1989). Skipping Slots in GenevaWheels. DET - Colloquium, “Leer der Mechanismen”,.[189]

98.(DET) Schickendantz, L. D. (1989). Bewegingssimulatie en Botsingsdetektie bij Mechanismen. DET - Colloquium, “Leer der Mechanismen”,.[190]

99.(DELT) Niens, O.F.M. (1994). TADSOL integratie in Unigraphics II. Delt Colloquium, 6p..[201]

100.Dhande, S. G. and J. Chakraborty (1976). Analysis of Profiled-follower Mechanisms. Mechanism and Machine Theory, 11, 131-139.[32]

101.Dhande, S. G. and J. Chakraborty (1975). Mechanical Error Analysis of Cam-Follower Systems - A Stochastic Approach. J. Mech. E., 957-962.[17] 102.Di Benedetto, A. and A. Vinciguerra (1982). Kinematic Analysis of Plate Cam Profiles Not Analytically Defined. Transactions of the ASME, Journal of Mechanical Design 104(1), 34-38.

103.Di Benedetto, A. and A. Vinciguerra (1979). A New Algorithm for Kinematic Synthesis of Plate Cam Profiles for Prescribed Follower Acceleration. In Proceedings of the Fifth World Congress on the Theory of Machines and Mechanisms, Montreal, Canada, pp. 549-552.

104.Di Benedeto, A. (1975). Some Methods of Kinematic Syntheses of Cam Profiles for Prescribed Jerk Pattern. J. Mech. E., 963-968.[15] 
105.Dierckx, P. (1993). Curve and Surface Fitting with Splines (smoothing with end point derivative constraints - part of the book), Clarendon Press, Oxford.[192]

106.Dierckx, P. (1979). Het aanpassen van krommen en oppervlakken aan meetpunten met behulp van splinefunkties. (part of doctorate dissertation).[191]

107.Dierckx, P. (1978). Het vereffenen van diskrete meetgegevens met behulp van splinefunkties die aan zekere randvoorwaarden voldoen. 10p..[205]

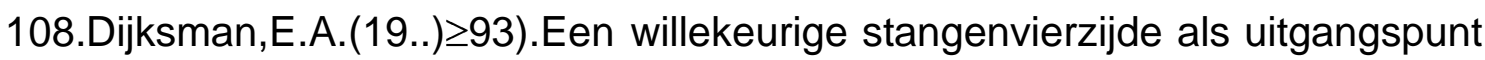
voor het ontwerpen van exacte rechtgeleidingen (Inversiemechanismen). 30p..[200]

109.Dinsdale, J. and A. J. Hutcheon (1993). Self-Service Banking and Mechatronics: The First Twenty Years. Proc. Instn. Mech. Engrs. 207(C), 1-8. 110.Dittrich, G. (19..). KUBER Programm-System zur Rechnerunterstützten Konstruktion von Kurven- und Kurven-Kurbelgetrieben. Aachen.[97b-_]

111.Dixon, M. W. and K. L. Ting (1979). Generalized Design Technique for a Spatial Cam-Link Function Generator. In Proceedings of the Fifth World Congress on the Theory of Machines and Mechanisms, Montreal, Canada, pp. 1307-1310.

112.Dooner, D. B. (1997). Use of Noncircular Gears to Reduce Torque and Speed Fluctuations in Rotating Shafts. Transactions of the ASME, Journal of Mechanical Design 119(2), 299-306.

113.Dresig, H. et alli. (1990). Drehzahlsteigerung von Kurvengetrieben mit elastischem An- und Abtrieb durch optimale Profile. VDI Bericthe nr.847, 201-215.[155] 
114.Dresner, T. L. and P. Barkan (1995). New Methods for the Dynamic Analysis of Flexible Single-Input and Multi-Input Cam-Follower Systems. Transactions of the ASME, Journal of Mechanical Design 117(1), 150-155.

115.Druce, G. (1976). Cams and Followers. Engineering,Technical file no.25, 110.[46]

116.Duca, C. D. and I. Simionescu (1980). The Exact Synthesis of Single-Disk Cams with Two Oscillating Rigidly Connected Roller Followers. Mechanism and Machine Theory 15(3), 213-220.

117.Dudley, W. M. (1948). New Methods in Cam Design. Trans. SAE 2(1), January, pp. 1933.

118.Dudley, W. M. (1947). A New Approach to Cam Design. Machine Design $19\left(\_\right)$, July _, 143-148, 184.

119.Eiss, Jr., N. S. (1964). Vibrations of Cams Having Two Degrees of Freedom. Transactions of the ASME, Journal of Engineering for Industry 86(4), 343-350.

120.El-Shakery, S. A. and Y. Terauchi (1984). A Computer-Aided Method for Optimum Design of Plate Cam-Size Avoiding Undercutting and Separation Phenomena-II: Design Nomograms.Mechanism and Machine Theory 19(2), 235-241.

121.Ephanov, A. V. and Y. Hurmuzlu (1997). Implementation of Sensory Feedback and Trajectory Tracking in Active Telemanipulation Systems. Transactions of the ASME, Journal of Dynamic Systems, Measurement, and Control 119(3), 447-454. 
122. Erdman, A. G. and G. N. Sandor (1972). Kineto-Elastodynamics - A Review of the State of the Art and Trends. Mechanism and Machine Theory 7(1), 1933.

123.Eumurian, C. (1960). A new method for Designing Cams for Analog Computers. 'Design-Data Sheet', October 27, 145, 146, 152-154.[66]

124.Fabien, B. C., R. W. Longman, and F. Freudenstein (1994). The Design of High-Speed Dwell-Rise-Dwell Cams Using Linear Quadratic Optimal Control Theory. Transactions of the ASME, Journal of Mechanical Design 116(3), 867-874.

125.Fenton, R. G. (1975). Optimum Design of Disc Cams. J. Mech. E., 781784.[30]

126.Fenton, R. G. (1966). Determining Minimum Cam Size. Machine Design, January 20, 155-158.[55]

127.Fenton, R. G. (1966). Reducing Noise in Cams. Machine Design, April 14, 187-190.[88a-_]

128.Ferguson, R. J., L. F. Daws and J. H. Kerr (1975). The Design of a Stepless Transmission Using Non-Circular Gears. Mechanism and Machine Theory 10(6), 467-478.

129.Gupta, K. C. (1977). A Note on the Optimum Design of Four Bar CrankRocker Mechanisms. Mechanism and Machine Theory 12(3), 247-254.

130.Flugrad, Jr., D. R. (1986). Cam Synthesis and Analysis for an Elastic Follower Linkage. Transactions of the ASME, Journal of Mechanisms, Transmissions, and Automation in Design 108(3), 367-372. 
131.Fosdick, R. and Y. Ketema (1998). A Thermoviscoelastic Dynamic Vibration Absorber. Transactions of the ASME, Journal of Applied Mechanics 65(1), 17-24.

132.Freudenstein, F. (1984). Machine Dynamics: Some Thoughts on Research Initiatives. Transactions of the ASME, Journal of Mechanisms, Transmissions, and Automation in Design 106(3), 264-266.

133.Freudenstein, F., L. W. Tsai, and E. R. Maki (1984). The Generalized

Oldham Coupling. Transactions of the ASME, Journal of Mechanisms, Transmissions, and Automation in Design 106(4), 475-481.

134.Freudenstein, F., M. Mayourian, and E. R. Maki (1983). Energy Efficient Cam-Follower Systems. Transactions of the ASME, Journal of Mechanisms, Transmissions, and Automation in Design 105(4), 681-685.

135.Fritzen, C.-P (1986). Identification of Mass, Damping, and Stiffness Matrices of Mechanical Systems. Transactions of the ASME, Journal of Vibration, Acoustics, Stress, and Reliability in Design 108(1), 9-16.

136.Ge, Q. J. and J. Rastegar (1996). Bernstein-Bézier Harmonics and Their Applications to Robot Trajectory Synthesis With Minimal Vibrational Excitation. Transactions of the ASME, Journal of Mechanical Design 118(4), $509-514$.

137.Gehlsdorf, W. et alli. (1983). Maschinelle Programmierung von NCMaschinen. Internationalen Kongresses Metallbearbeitung IKM 82, ZwF78, 20-24.[93-_-c]

138.Gentner, K. (1991). Flexible Milling and Grinding of Camshafts. Industrial \& Production Engineering, 15(4).[163] 
139.Gierse, F. J. (1983). Konstruktive Gestaltung von Kurvengetrieben. Begleittext zum Vortrag am 22.6.1983 vor dem VDI-AKE des Aachener Bezirksvereins, 69p..[86_-b]

140.Gierse, F. J. (1979). MeBtechnik in der getriebetechnischen Praxis. VDIBerichte Nr.321, .[83_-b]

141.Giordana, F., V. Rognoni, and G. Ruggieri (1980). The Influence of Construction Errors in the Law of Motion of Cam Mechanisms. Mechanism and Machine Theory 15(1), 29-45.

142.Ghosh, A. and R. P. Yadav (1983). Synthesis of Cam-Follower Systems With Rolling Contact. Mechanism and Machine Theory 18(1), 49-56.

143.González-Palacios, M. A. and J. Angeles (1994). The Generation of Contact Surfaces of Indexing Cam Mechanisms - A Unified Approach. Transactions of the ASME, Journal of Mechanical Design 116(2), 369-374.

144.González-Palacios, M. A. and J. Angeles (1994). Synthesis of Contact Surfaces of Spherical Cam-Oscillating Roller-Follower Mechanisms: A General Approach. Transactions of the ASME, Journal of Mechanical Design 116(1), 315-319.

145.Grace, A. (19..). Optimization TOOLBOX, for use with MATLAB..[210] 146.Gupta, K. C. (1997). Measures of Positional Error for a Rigid Body. Transactions of the ASME, Journal of Mechanical Design 119(3), 346-348.

147.Gupta, K. C. and J. L. Wiederrich (1983). Development of Cam Profiles Using the Convolution Operator. Transactions of the ASME, Journal of Mechanisms, Transmissions, and Automation in Design 105(4), 654-657.

148. Hadekel, R. (1978). Converting Linear to Rotary Motion with Cross-Link Mechanisms. Machine Design, July 20, 64-67.[11] 
149. Haessig Jr., D. A. and B. Friedland (1991). On the Modeling and Simulation of Friction. Transactions of the ASME, Journal of Dynamic Systems, Measurement, and Control 113(3), 354-362.

150.Hain, K. (1978). Entwurf ubertragungsgungstiger Zylinderkurven-und Kegelkurven-Getriebe. Werkstatt und Betrieb, 111(2).[33]

151.Han, J. T. (1978). A Computer-Aided Feel Cam Design. Trans. ASME, J. Mech. Design, 100, 604-606.[36]

152.Hanafi, A., F. W. Wright, and J. R. Hewit (1984). Optimal Trajectory Control of Robotic Manipulators. Mechanism and Machine Theory 19(2), 267-273.

153. Hatwal, H. and A. Ghosh (1979). Dynamics of a Three-Wheeled Vehicle. In Proceedings of the Fifth World Congress on the Theory of Machines and Mechanisms, Montreal, Canada, pp. 930-934.

154.Hazelrigg, G. A. (1997). On Irrationality in Engineering Design. Transactions of the ASME, Journal of Mechanical Design 119(2), 194196.Allais, D. C. (1963). Cycloidal vs Modified Trapezoidal Cams-In Elastic Systems, which Profile Minimizes Acceleration at the Driven Mass?. Machine Design, Jan. 31, 5p..[56]

155.Hebeler, C. B. (1961). Design equations and graphs for finding the dynamic response of ...Cycloidal-Motion Cam Systems. Machine Design, February 2, 102-107.[89a-_]

156. Heck, B. S. and A. A. Ferri (1996). Model Reduction of Coulomb Friction Damped Systems Using Singular Perturbation Theory. Transactions of the ASME, Journal of Dynamic Systems, Measurement, and Control 118(1), 8491. 
157.Hernandez-G. I. and B. Arroyo-R. (1991). Kinematics of Constant Speed Grinding of Planar Complex Shape Pieces. Eighth World Congress on the Theory of Machines and Mechanisms, Prague, Czechoslovakia, 945947.[165]

158. Hockey, B. A. (1972). The Minimization of the Fluctuation of Input-Shaft Torque in Plane Mechanisms. Mechanism and Machine Theory 7(3), 335346.

159.Holka, H. (1979). A Dynamic Vibration Damper With an Active Frequency Tuning. In Proceedings of the Fifth World Congress on the Theory of Machines and Mechanisms, Montreal, Canada, pp. 333-336.

160.Howell, L. L., A. Midha, and T. W. Norton (1996). Evaluation of Equivalent Spring Stiffness for Use in a Pseudo-Rigid-Body Model of Large-Deflection Compliant Mechanisms. Transactions of the ASME, Journal of Mechanical Design 118(1), 126-131.

161. Howell, L. L. and A. Midha (1996). A Loop-Closure Theory for the Analysis and Synthesis of Compliant Mechanisms. Transactions of the ASME, Journal of Mechanical Design 118(1), 121-125.

162. Howell, L. L. and A. Midha (1994). A Method for the Design of Compliant Mechanisms With Small-Length Flexural Pivots. Transactions of the ASME, Journal of Mechanical Design 116(1), 280-290.

163.Hsu, W. and A. P. Pisano (1996). Modeling of a Finger-Follower Cam System With Verification in Contact Forces. Transactions of the ASME, Journal of Mechanical Design 118(1), 132-137.

164.Hsu, M. H., H. S. Yan, and M. C. Tsai (1995). An Experimental Study of the Effects of Cam Speeds on Cam-Follower Systems. In Proceedings of the 
Ninth World Congress on the Theory of Machines and Mechanisms, Milan, Italy, pp. 759-764.

165.Hunt, K. H. (1973). Profiled-Follower Mechanisms. Mechanism and Machine Theory 8(3), 371-395.

166. IBM Application Program (1969). Mechanism Design System-Kinematics / Application Description Manual. IBM, Second Edition, H20-0493-1, April, 23p..[89_-_-c]

167.Inoue, J., Y. Araki, and S. Miyaura (1979). On The Self-Synchronization of Mechanical Vibrator Due to Impact. In Proceedings of the Fifth World Congress on the Theory of Machines and Mechanisms, Montreal, Canada, pp. 420-423.

168.James, P. A. and B. Roth (1994). A Unified Theory for Kinematic Synthesis. Transactions of the ASME, Journal of Mechanical Design 116(1), 144-154. 169.Jasinski, P. W., H. C. Lee, and G. N. Sandor (1971). Vibrations of Elastic Connecting Rod of a High-Speed Slider-Crank Mechanism. Transactions of the ASME, Journal of Engineering for Industry 93(2), 636-644.

170.Jasinski, P. W., H. C. Lee, and G. N. Sandor (1970). Stability and SteadyState Vibrations in a High-Speed Slider-Crank Mechanism. Transactions of the ASME, Journal of Applied Mechanics 37(4), 1069-1076.

171.Jeans, H. (1957). Designing Cam Profiles with digital computers. Machine Design, October 31, 103-106.[65]

172.Jensen, P. W. (1965). Cam Design and Manufacture. New York: Industrial Press. 
173.Jezequel, L. (1986). Three New Methods of Modal Identification. Transactions of the ASME, Journal of Vibration, Acoustics, Stress, and Reliability in Design 108(1), 17-25.

174.Jhala, P. B. and C. G. Venkataramanan (1979). Optimisation of Picking Cam Design Through Dynamic Studies. In Proceedings of the Fifth World Congress on the Theory of Machines and Mechanisms, Montreal, Canada, pp. 992-995.

175.Johnson, R. C. (1984). Force Reduction by Motion Design in Spring-

Loaded Cam Mechanisms. Transactions of the ASME, Journal of Mechanisms, Transmissions, and Automation in Design 106(3), 278-284.

176.Johnson, R. C. (1959). The Dynamic Analysis and Design of Relatively Flexible Cam Mechanisms Having More Than One Degree of Freedom. Transactions of the ASME, Journal of Engineering for Industry 81(4), 323331.

177.Johnson R. C. (1959). A simple numerical method for dynamic analysis and design of Flexible Cam Mechanisms. Machine Design, September 3, 6p..[63] 178.Johnson, R. C. (1958). Impact Forces in Mechanisms. Machine Design 30(12), June 12, 138-146.

179.Johnson, R. C. (1957). How Profile Errors Affect CAM DYNAMICS. Machine Design, February 7, 105-108.[60]

180.Johnson, R. C. (1957b). Method of finite differences in cam design. Machine Design 29(23), November 14, 105-108.

181.Johnson, R. C. (1956). How to design high-speed CAM MECHANISMS for optimum cam and follower proportions. Machine Design, January 26, 8589.[59] 
182.Johnson, R. C. (1956). A new point of view on Minimizing Cam Vibrations. Machine Design, August 9, 103-104.[62]

183.Johnson, R. C. (1956). A rapid method for developing CAM PROFILES having desired acceleration characteristics. Machine Design, Dec. 13, 129132.[57]

184.Johnson, R. C. (1955). Method of finite differences provides simple but flexible arithmetical techniques for CAM DESIGN. Machine Design, November 195-204.[58]

185.Kahraman, A. and G. W. Blankenship (1997). Experiments on Nonlinear Dynamic Behavior of an Oscillator With Clearance and Periodically TimeVarying Parameters. Transactions of the ASME, Journal of Applied Mechanics 64(1), 217-226.

186.Kanango, R. N. and N. Patnaik (1979). Improving Dynamic Characteristics of a Cam-Follower Mechanism Through Finite Difference. In Proceedings of the Fifth World Congress on the Theory of Machines and Mechanisms, Montreal, Canada, pp. 591-594.

187.Kanarachos, A. (1977). Zur Auslegung von Kurvengetrieben unter Beruck__sichtigung des dynamischen Verhaltens. Konstruktion 29(H.5), 188190.[177]

188.Kanzaki, K., N. Kobayasi, and H. Kawasaki (1995). Output of Cam Motion Curve by DC Servo Motor and High Speed Positioning. In Proceedings of the Ninth World Congress on the Theory of Machines and Mechanisms, Milan, Italy, pp. 739-743. 
189.Kanzaki, K. and K. Itao (1972). Polydyne Cam Mechanisms for Typehead Positioning. Transactions of the ASME, Journal of Engineering for Industry 94(1), 250-254.

190.Kaufman, R. E. (1978). Mechanism Design by Computer, Machine Design, October 26, 94-100.[10]

191.Kazerooni, H. and C. L. Moore (1997). An Approach to Telerobotic Manipulations. Transactions of the ASME, Journal of Dynamic Systems, Measurement, and Control 119(3), 431-438.

192.Kerle, H. (1977). Analoge MeBsynthese kinematischer und dynamischer KenngroBen bei Kurvengetrieben. Feinwerktechnik \& Messtechnik,85(8), 379-385.[83a-_]

193.Kim, H. R. and W. R. Newcombe (1982). The Effect of Cam Profile Errors and System Flexibility on Cam Mechanism Output. Mechanism and Machine Theory 17(1), 57-72.

194.Kim, H. R. and W. R. Newcombe (1982). The Effect of Cam Profile Errors and System Flexibility on Cam Mechanism Output. Mechanism and Machine Theory, 17(1), 57-72.[28]

195.Kim, H. R. and W. R. Newcombe (1978). Stochastic Error Analysis in Cam Mechanisms Mechanism and Machine Theory, 13, 631-641.[50]

196.Kiper, G. (1979). Ebene kinematische Ketten mit ein und zwei Laufgraden sowie bis zu sechs Gliedern und nichtzwanglaufigen Kurvengelenken. Konstruktion, 31(3), 111-115.[53]

197.Kirmse, W. and P. Johannsen (1982). Regelung der WerkstuckDrehfrequenz beim Nockenschleifen. Zeitschrift fur Maschinenbau, Konstruktion und Fertigung, Werkstatt und Betrieb, 115(3), 149-155.[94b-] 
198.Koetsier, T. (1986). From Kinematically Generated Curves To Instantaneous Invariants: Episodes in the History of Instantaneous Planar Kinematics. Mechanisms and Machine Theory, 21(6), 489-498.[197] 199.Koetsier, T. (1983). A Contribution to the History of Kinematics-I: Watt's Straight-Line Linkages and the Early French Contributions to the Theory of the Planar 4-Bar Coupler Curve. Mechanism and Machine Theory, Vol. 18(1), 37-42.[91-_-c]

- Koetsier, T. (1983). A Contribution to the History of Kinematics-II: The Work of English Mathematicians on Linkages During the Period 1869-78. Mechanism and Machine Theory, Vol. 18(1), 43-48.[91-_-c]

200.Konietzko, P. (1978). Computergesteuerte Kurvenfrasmaschine. Werkstatt und Betrieb, 111(12), 807-808.[77]

201.Koster, M. P. (1975). Effect of Flexibility of Driving Shaft on the Dynamic Behavior of a Cam Mechanism. Trans. ASME, J. Eng. for Industry, May, 595-602.[70]

202.Koster, M. P. (1975). Effect of Flexibility of Driving Shaft on the Dynamic Behavior of a Cam Mechanism. Transactions of the ASME, Journal of Engineering for Industry 97(2), 595-602.

203.Koster, M. P. (1974). Vibrations of Cam Mechanisms. London: The Macmillan Press Ltd.

204.Koster, M. P. (19..). The Effects of Backlash and Shaft Flexibility on the Dynamic Behaviour of a Cam Mechanism. N.V.Philips, Eindhoven, The Netherlands, 25p..[73]

205.Kota, S. and A. G. Erdman (1997). Motion control in product design. Mechanical Engineering 119(8), 74-77. 
206.Kota, S. and S. Bidare (1997). Systhematic Synthesis and Applications of Novel Multi-Degree-of-Freedom Differential Systems. Transactions of the ASME, Journal of Mechanical Design 119(2), 284-291.

207.Kota, S., G. K. Ananthasuresh, S. B. Crary, and K. D. Wise (1994). Design and Fabrication of Microelectromechanical Systems. Transactions of the ASME, Journal of Mechanical Design 116(4), 1081-1088.

208.Koumans, P. W. (1982). Praktische ontwerpmethode voor toepassing in draaitafels TOROIDE NOKKEN. PT/Werktuigbouw 37(5), 60-65.[92b-]

209.Koumans,P.W. (1976). Een Numeriek Bestuurde Machine voor het Frezen van Nokken. PT Overdurk uit pr/werktuigbouw, 31(12), 11p..[76]

210.Kozanek, J. (19..). Identification of complex non-conservative continua -Kozanek, J. (1987). The Qualification Number of a Complex Vector. Mech. Mach. Theory,22(4), 391-392.

211.--Kozanek, J. (1983). Dynamic Investigation of Rotors. Proc. XIV Conf. Dynamics of Machines, 227-228.

-Kozanek, J. (1991). On Identification of Evolutive Dynamic Systems. Eighth World Congress on the Theory of Machines and Mechanisms (IFTOMM), 6 , $4 p . .[156]$

212.Kozhevnikov, S. N., E. Ya. Antonyuk and A. I. Tkachuk (1974). Synthesis of a Cam-Differential Mechanism with Periodic Dwell of the Output Link. Mechanism and Machine Theory 9(2), 219-229.

213.Kraver, T. C., G. W. Fan, and J. J. Shah (1996). Complex Modal Analysis of a Flat Belt Pulley System With Belt Damping and Coulomb-Damped Tensioner. Transactions of the ASME, Journal of Mechanical Design 118(2), 306-311. 
214.Kunad, G. et alli. (1978). Lage-, Geschwindigkeits- und Beschleunigungsanalyse Raumlicher Mechanismen. German, October.[104] 215.Kunad, G. and Goetze, R. (1978). Kinetostatische Analyse Raumlicher Mechanismen. German, October.[105]

216.Kunad, G. and J. Nothdurft (1978). Raumliche Mechanismen Mit Elastischen Gliedern und Federn-Ermittlung Stabiler Gleichgewichtslagen.German, Oct.[106]

217.Kupka, M. (1983). Schwingungsarme Kurvengetriebe mit Gleichdicken. VDI-Berichte, Nr. 489.[98--c]

218.Lakshminarayana, K. and K. S. Tripathi (1991). Roller-to-Cam Type Intermittent Motion Mechanisms: Optimal Designs and Capability Limits. In Proceedings of the Eighth World Congress on the Theory of Machines and Mechanisms, Prague, Czechoslovakia, pp. 691-694.

219.Lal, J. S. (1979). Optimum Design of Linkages Using Fourier Series and Taylor Series. In Proceedings of the Fifth World Congress on the Theory of Machines and Mechanisms, Montreal, Canada, pp. 1004-1007.

220.Lee, C.-T., S. W. Shaw, and V. T. Coppola (1997). A Subharmonic Vibration Absorber for Rotating Machinery. Transactions of the ASME, Journal of Vibration and Acoustics 119(4), 590-595.

221.Lee, T. W. and C. Cheng (1984). Optimum Balancing of Combined Shaking Force, Shaking Moment, and Torque Fluctuations in High-Speed Linkages. Transactions of the ASME, Journal of Mechanisms, Transmissions, and Automation in Design 106(2), 242-251.

222.Lee, D. (1982). Constant-Torque Spring Linkages. Machine Design, May 6, 103-104.[89_-b-_] 
223.Leuridan, J. M., D. L. Brown, and R. J. Allemang (1986). Time Domain Parameter Identification Methods for Linear Modal Analysis: A Unifying Approach. Transactions of the ASME, Journal of Vibration, Acoustics, Stress, and Reliability in Design 108(1), 1-8.

224.Liang, J.-W. and B. F. Feeny (1998). Dynamical Friction Behavior in a Forced Oscillator With a Compliant Contact. Transactions of the ASME, Journal of Applied Mechanics 65(1), 250-257.

225.Liao, C. Y. and C. K. Sung (1993). An Elastodynamic Analysis and Control of Flexible Linkages Using Piezoceramic Sensors and Actuators. Transactions of the ASME, Journal of Mechanical Design 115(3), 658-665.

226.Lilov, L. (1985). Bewegungsgleichungen elastischer Mehrkorpersysteme. ZAMM - Z. Angew. Math. u. Mech. 65(4), 74-76.[158]

227.Lilov, L. (1984). Variationsprinzipien in der Starrkorpermechanik. ZAMM 64, 377-379.

228.Liu, S.-W. and T. Singh (1997). Robust Time-Optimal Control of Flexible Structures With Parametric Uncertainty. Transactions of the ASME, Journal of Dynamic Systems, Measurement, and Control 119(4), 743-748.

229.Liu, H.-T. J. (1997). Synthesis and Steady-State Analysis of High-Speed Elastic Cam-Actuated Linkages With Fluctuated Speeds by a Finite Element Method. Transactions of the ASME, Journal of Mechanical Design 119(3), 395-402.

230.Liu, J.-Y. and H.-S. Yan (1994). Surface Geometry of Variable Pitch Cylindrical Cams with Conical Meshing Elements. Transactions of the ASME, Journal of Mechanical Design 116(3), 862-866. 
231.Liu, H.-T. J. and D. R. Flugrad, Jr. (1993). Synthesis and Steady State Analysis of High-Speed Elastic Cam-Actuated Linkages by a Finite Element Method. Transactions of the ASME, Journal of Mechanical Design 115(4), 800-807.

232.Lo, C. and P. Y. Papalambros (1996). A Convex Cutting Plane Algorithm for Global Solution of Generalized Polynomial Optimal Design Models. Transactions of the ASME, Journal of Mechanical Design 118(1), 82-88.

233.Lo, C. and P. Y. Papalambros (1996). A Deterministic Global Design Optimization Method for Nonconvex Generalized Polynomial Problems. Transactions of the ASME, Journal of Mechanical Design 118(1), 75-81.

234.Lo, C. and P. Y. Papalambros (1995). On Global Feasible Search for Global Design Optimization with Application to Generalized Polynomial Models. Transactions of the ASME, Journal of Mechanical Design 117(3), $402-408$.

235.Loeff, L. and A. H. Soni (1975). Optimum Sizing of Planar Cams. J. Mech. E., 777-780.[16]

236.López-Cajún, C. S., J. Angeles, and C. Fortin (1988). The Computer Integration of the Optimum Design and Manufacture of Cam Mechanisms. $7 p . .[149]$ 237.Lord, P. R. and M. H. Mohamed (1975). An Analysis of the Picking Mechanism of a Textile Loom. Transactions of the ASME, Journal of Engineering for Industry 97(2), 385-390.

238.Lowen, G. G. and W. G. Jandrasits (1972). Survey of Investigations into the Dynamic Behavior of Mechanisms Containing Links with Distributed Mass and Elasticity. Mechanism and Machine Theory 7(1), 3-17. 
239.Luo, H. and S. Hanagud (1998). On the Dynamics of Vibration Absorbers With Motion-Limiting Stops. Transactions of the ASME, Journal of Applied Mechanics 65(1), 223-233.

240.Lynch, A. G. and M. J. Vanderploeg (1995). A Symbolic Formulation for Linearization of Multibody Equations of Motion. Transactions of the ASME, Journal of Mechanical Design 117(3), 441-445.

241.Mabie, H. H. and C. F. Reinholtz (1987). Mechanisms and Dynamics of Machinery. New York: John Wiley \& Sons.

242.MacCarthy, B. L., J. M. Sharp, and N. D. Burns (1992). An Analysis of the Mechanical Forces in Latch Needle Cam Systems. Proc. Instn. Mech. Engrs. 206(C), 129-137.

243.MacCarthy, B. L. (1988). Quintic splines for kinematic design. Computeraided Design, 20(7), 406-415.[148]

244.Mahyuddin, A. I. and A. Midha (1994). Influence of Varying Cam Profile and Follower Motion Event Types on Parametric Vibrations and Stability of Flexible Cam-Follower Systems. Transactions of the ASME, Journal of Mechanical Design 116(1), 298-305.

245.Mahyuddin, A. I., A. Midha, and A. K. Bajaj (1994). Evaluation of Parametric Vibration and Stability of Flexible Cam-Follower Systems. Transactions of the ASME, Journal of Mechanical Design 116(1), 291-297.

246.Mank, K. D. (1978). Zur MaBsynthese von ebenen, viergliedrigen Getrieben mit infolge Formschlusses zwanglaufigen Kurvengelenken. Konstruktion, 30(3), 94-98.[80a-x]

247.(Malkin) Gal-Tzur, Z., M. Shpitalni, and S. Malkin (1986). Integrated CAD/CAM System for Cams. Annals of the CIRP, 35(1), 99-102.[124] 
248.(Manolescu) Nitescu, P. N. and N. I. Manolescu (1980). On the Structural Synthesis and Kinematic Analysis of Open-Loop Manipulation. Mechanism and Machine Theory, 15(4-E), 295-317.[116]

249.Manolescu, N. I., D. Stanescu, and G. Bonoci (1979). The Optimization of the Cams Utilised in the Construction of the Machine-Tools. In Proceedings of the Fifth World Congress on the Theory of Machines and Mechanisms, Montreal, Canada, pp. 1295-1298.

250.(Manolescu) Ionescu, T. and N. I. Manolescu (19..)( $\geq 79$ ). System Analysis and Structural Criteria Concerning Diesel Locomotives. ASME paper: 80$D E T-105,10 p . .[114]$

251. Manolescu, N. I. (1979). A unified method for the formation of all planar jointed kinematic chains and Baranov trusses. Pion Publication, (printed in Great Britain), 447-454.[115]

252.Mansour, W. M. (1979). Dynamics and Stability of an Orientation Mechanism for Submersibles. In Proceedings of the Fifth World Congress on the Theory of Machines and Mechanisms, Montreal, Canada, pp. 579-582.

253.Marinov, P., S. Pavlov, and V. Draganov (1994). Efficiency of a Multiple Flywheel Recuperation System. Transactions of the ASME, Journal of Mechanical Design 116(1), 332-336.

254.Marracino, C. R. (1978). Four-slides, Part 5 - Cam Engineering. Manufacturing Engineering, June, 60-65.[9]

255.Maschinenbautechnik (1984). $\quad$-Berlin, Mai $\quad-\quad(4$ articles)[120] $\begin{array}{llll}\text {--Muller, } & \text { J., } & \text { Hochschullehrfilm } & \text { Kurvengetriebe }\end{array}$

--Dresig, H. and Rossler, J., Bewegungsgesetz schwingungsarmer Kurvengetriebe --Glaubitz, W. and Lunze, U., Zur Auswertung von 
Kurvenscheibenmessungen

auf

KoordinatenmeBgeraten

--Tilgner, W., SerienmaBige Instandsetzung von Nockenwellen..[120a,b,c,d] 256.Matthew, G. K. (1979). The Modified Polynomial Specification for Cams. In

Proceedings of the Fifth World Congress on the Theory of Machines and Mechanisms, Montreal, Canada, pp. 1299-1302.

257.Matthew, G. K. and D. Tesar (1976). Cam System Design: The Dynamic Synthesis and Analysis of the One Degree of Freedom Model. Mechanism and Machine Theory, 11, 247-257.[145]

258. Matthew, G. K. and D. Tesar (1975). The Design of Modeled Cam Systems

- Part I: Dynamic Syntheses and Chart Design For the Two-Degree-of-

Freedom Model and Part II: Minimization of Motion Distortion Due to Modeling Errors. Trans. ASME, J. Eng. Ind., Nov.,1175-1189.[40]

259.Matthew, G. K. and D. Tesar (1975). The Design of Modeled Cam Systems

- Part II: Minimization of Motion Distortion Due to Modeling Errors. Transactions of the ASME, Journal of Engineering for Industry 97(4), 11811189.

260.Matthew, G. K. and D. Tesar (1975). The Design of Modeled Cam Systems

- Part I: Dynamic Synthesis and Chart Design for the Two-Degree-ofFreedom Model. Transactions of the ASME, Journal of Engineering for Industry 97(4), 1181-1189.

261.Maunder, L. (1979). Theory and Practice - Keynote Lecture. In Proceedings of the Fifth World Congress on the Theory of Machines and Mechanisms, Montreal, Canada, pp. 1-8. 
262.Maunder, L. (1979). Dynamically Tuned Gyroscopes. In Proceedings of the Fifth World Congress on the Theory of Machines and Mechanisms, Montreal, Canada, pp. 470-473.

263.May, H. O. (1984). Nichtdifferenzierbare Funktionen in der Analytischen Mechanik. ZAMM 64, 379-380.[159]

264.Mayne, R. W., J. P. Sadler, and K. C. Fan (1980). Generalized Study of Crank-Rocker Mechanisms Driven by a d.c. Motor. Part II: Applications. Mechanism and Machine Theory 15(6), 447-461.

265.McGarva, J. R. (1995). Concept and Improvement of Cam Mechanisms Using CAD. In Proceedings of the Ninth World Congress on the Theory of Machines and Mechanisms, Milan, Italy, pp. 735-738.

266.Mentrasti, L. (1998). Paradoxes in Rigid-Body Kinematics of Structures.

Transactions of the ASME, Journal of Applied Mechanics 65(1), 218-222.

267.Mercer, Jr., S. and A. R. Holowenko (1958). Dynamic Characteristics of Cam Forms Calculated by the Digital Computer. Transactions of the ASME, Journal of Engineering for Industry 80(4), 1696-1705.

268.Merkel, P. (1992)(?). Exakt im Schritt. Industrie Anzeiger, 47, 2p..[199] 269.Meusburger, P. (1991). Computer-controlled Non-cylindrical Grinding of Eccentric Workpieces. Industrial\&Production Engineering, Metalworking, 15(2), 50-58.[147]

270.Michelena, N. F. and P. Y. Papalambros (1995). Optimal Model-Based Decomposition of Powertrain System Design. Transactions of the ASME, Journal of Mechanical Design 117(4), 499-505. 
271.Midha, A. and D. A. Turcic (1980). On the Periodic Response of Cam Mechanism With Flexible Follower and Camshaft. Transactions of the ASME, Journal of Dynamic Systems, Measurement, and Control 102(4), 255-264.

272.Midha, A., M. L. Badlani, and A. G. Erdman (1979). Periodic Response of High-Speed Cam Mechanism with Flexible Follower and Camshaft Using a Closed-Form Numerical Algorithm. In Proceedings of the Fifth World Congress on the Theory of Machines and Mechanisms, Montreal, Canada, pp. 1311-1314.

273.Miksch-Machinen, Erlauterungen. 24p..[88_-b]

274. Miladinovic, L. (1995). Computer Aided Analysis of Planar Cam Mechanisms. In Proceedings of the Ninth World Congress on the Theory of Machines and Mechanisms, Milan, Italy, pp. 706-708.

275.Mitchiner, R. G. and H. H. Mabie (1977). The Synthesis of 4-bar Linkage Coupler Curves using Derivatives of the Radius of Curvature-II. Circular Path Procedure. Mechanism and Machine Theory, 12(2-b), 147-163.[103] 276.Miu, D. K. (1991). Physical Interpretation of Transfer Function Zeros for Simple Control Systems With Mechanical Flexibilities. Transactions of the ASME, Journal of Dynamic Systems, Measurement, and Control 113(3), 419424.

277.Molian, S. (1973). Mechanisms with Discontinuous Transmission Ratios. Mechanism and Machine Theory 8(3), 365-369.

278.Molian, S. (1968). The Design of Cam Mechanisms and Linkages. New York: American Elsevier. 
279.Moura, J. T., H. Elmali, and N. Olgac (1997). Sliding Mode Control With Sliding Perturbation Observer. Transactions of the ASME, Journal of Dynamic Systems, Measurement, and Control 119(4), 657-665.

280.Moustafa, K. A. F. and A. M. Ebeid (1988). Nonlinear Modeling and Control of Overhead Crane Load Sway. Transactions of the ASME, Journal of Dynamic Systems, Measurement, and Control 110(2), 266-271.

281.Moustafa, M. A. (1977). The Minimum Base Circle Radius for Plane Cams. Journal of Eng. Sci., 3(2), 43-52.[47]

282.Muller, J. (1990). Getriebetechnische Lehre und Forschung an der Universitat Rostock - ein Jubilaumsruckblick. Maschinenbautechnik, 39(9), $417-420 \cdot[174]$

283.Muller, J. (1990). 25 Jahre getriebetechnische Ausbildung an der alma mater rostociensis. Wiss. Z. UNI ROSTOCK - N-Reihe 39(6), 5-25.[162]

284.Muller, J. (1988). Zur Entwicklungsgeschichte der Kurvengetriebe. Wiss. Z. WPU - Rostock - N-Reihe 37(7), 56-88.[175]

285.Muller, J. (1984). Hochschullehrfilm Kurvengetriebe. Maschinenbautechnik, 33(5).[131] (=118b)

286.Muller, J. (1981). Lehrmodellschrank Kurvengetriebe.

Maschinenbautechnik, 30(10).[132]

287.Muller, J. (1975). EinfluB fehlerhafter Eingriffsverhaltnisse auf den Bewengungsverlauf bei ebenen Kurvengetrieben. Wissenschaftliche Zeitschrift der Universitat Rostok, 24(4), 479-485.[126]

288.Muller, J. (1969). Begriffe fur Kurvengetriebe. Maschinenbautechnik (Getriebetechnik) 18(9), 489-493.[127?] 
289.Muller, J. (1966). MeBtechnische Ermittlung und EinfluB der Fertigungsungenauigkeiten bei Kurvengetrieben. Industrie-Anzeiger, Essen, 88(40), May 20, 834-838.[134]

290.Mund, E. H., et alli. (1975). An Algorithm for the Interpolation of Functions Using Quintic Splines. Journal of Computational and Applied Mathematics, $\mathrm{I}(4) \cdot[171]$

291.Nadolski W. and A. Pierlorz (1979). Dynamic Analysis of Two-Mass Drive System. In Proceedings of the Fifth World Congress on the Theory of Machines and Mechanisms, Montreal, Canada, pp. 306-309.

292.Nath, P. K. and A. Ghosh (1980). Steady State Response of Mechanisms with Elastic Links by Finite Element Methods. Mechanism and Machine Theory 15(3), 199-211.

293.Nath, P. K. and A. Ghosh (1980). Kineto-Elastodynamic Analysis of Mechanisms by Finite Element Method. Mechanism and Machine Theory 15(3), 179-197.

294.Needle, S. A. and M. L. Hull (1997). An Off-Road Bicycle With Adjustable Suspension Kinematics. Transactions of the ASME, Journal of Mechanical Design 119(3), 370-375.

295.Neklutin, C. N. (1969). Mechanisms and Cams for Automatic Machines. New York: American Elsevier.

296.Nishioka, M. (1995). State of the Art of Torque Compensation Cam Mechanisms. In Proceedings of the Ninth World Congress on the Theory of Machines and Mechanisms, Milan, Italy, pp. 713-717. 
297.Nishioka, M. et alli. (1984). Development of Data Processing System for Cam and Linkage Mechanisms. International Symposium on Design and Synthesis, Tokio, July 11-13, 788-793.[118]

298.Nitao, J. J. and J. L. Wiederrich (1984). Some Extensions to an Algorithm for the Identification of Single-Degree-of-Freedom Machines. Transactions of the ASME, Journal of Mechanisms, Transmissions, and Automation in Design 106(4), 498-502.

299.Norton, R. L. (1993). Modern Kinematics: Developments in the Last Forty Years - Chapter 7: Cams and Cam Followers. Edited by A. G. Erdman, New York, John Wiley \& Sons.

300.Notash, L., R. G. Fenton, and J. K. Mills (1991). Optimal Design of Flexible Cam Mechanisms. In Proceedings of the Eighth World Congress on the Theory of Machines and Mechanisms, Prague, Czechoslovakia, pp. 695-698. 301.Noverraz, J. L. (19..). Qu'est-ce que la came argue?. La Revue Polytechnique, 1415, 1169.[27]

302.Novotny, J. (1991). Optimum Design and Accuracy of a Double Cam Mechanism. Eighth World Congress on the Theory of Machines and Mechanisms, Prague, Czechoslovakia, 699-702.[167]

303.Oblak, M., I. Ciglaric, and B. Butinar (1995). Optimal Design of Pushrod Actuated Valve Train Cam-Follower Mechanism. In Proceedings of the Ninth World Congress on the Theory of Machines and Mechanisms, Milan, Italy, pp. $754-758$.

304.Oderfeld, J. and A. Podorzelski (1991). On Designing Plane Cam Mechanisms. In Proceedings of the Eighth World Congress on the Theory of Machines and Mechanisms, Prague, Czechoslovakia, pp. 703-705. 
305.Oledzki, A. (1980). On the Modelling of Certain Mechanical Systems with a Hysteresis Loop. Mechanism and Machine Theory 15(6), 479-490. 306.Oledzki, A. (1973). Some Dynamic Properties of Systems with Cam Mechanisms. Mechanism and Machine Theory 8(4), 543-554.[107] 307.Oledzki, A. (1972). Dynamics of Piano Mechanisms. Mechanism and Machine Theory 7(4), 373-385.

308.Or, A. C. (1998). Chaotic Motions of a Dual-Spin Body. Transactions of the ASME, Journal of Applied Mechanics 65(1), 150-156.

309.Pagel, P. A. (1978). Sizing Cams for Long Life. Machine Design, Sept. 7.[48]

310.Pagel, P. A. (1978). Custom Cam from 'Building Blocks'. Machine Design, May 11.[49]

311.Pagilla, P. R. and M. Tomizuka (1997). Contact Transition Control of Nonlinear Mechanical Systems Subject to a Unilateral Constraint. Transactions of the ASME, Journal of Dynamic Systems, Measurement, and Control 119(4), 749-759.

312.Pandey, R. C. and A. Midha (1981). On Flexible Input Shaft and Flywheel Interaction In High-Speed Machines. Mechanism and Machine Theory 16(4), 321-337.

313.Pandian, S. R., Y. Hayakawa, Y. Kanazawa, Y. Kamoyama, and S. Kawamura (1997). Practical Design of a Sliding Mode Controller for Pneumatic Actuators. Transactions of the ASME, Journal of Dynamic Systems, Measurement, and Control 119(4), 666-674.

314.Park, T. and G. Z. Voyiadjis (1998). Kinematic Description of Damage. Transactions of the ASME, Journal of Applied Mechanics 65(1), 93-98. 
315.Parker, R. G. (1998). On the Eigenvalues and Critical Speed Stability of Gyroscopic Continua. Transactions of the ASME, Journal of Applied Mechanics 65(1), 134-140.

316.Paul, B. (1975). Analytical Dynamics of Mechanisms - A Computer Oriented Overview. Mechanism and Machine Theory 10(6), 481-507.

317.Paynter, H. M. (1980).Rufus Oldenburger Award (presentation by Donald N. Zwiep + response by Henry M. Paynter) + Microprocessors in Mechanical Engineering Education by P. M. Lynch + Survey of Microcomputer Education in Mechanical Engineering Departments by C. W. de Silva. Transactions of the ASME, Journal of Dynamic Systems, Measurement, and Control 102(2), 53-61.

318.Peters, D. A. (1979). The Effect of Rotation on Structural Stiffness. In Proceedings of the Fifth World Congress on the Theory of Machines and Mechanisms, Montreal, Canada, pp. 280-283.

319.Petersen, E. (1976). Kombinationsgesetze fur Bewegungen mit Rast-und Umkehrlagen. Konstruktion, 28, 90-96.[43]

320.Pisano, A. P. and H. T. Chen (1986). Coulomb Friction and Optimal Rocker Arm Ratio for High-Speed Cam Systems. Transactions of the ASME, Journal of Mechanisms, Transmissions, and Automation in Design 108(3), 340-344. 321.Pisano, A. P. (1984). Coulomb Friction in High-Speed Cam Systems. Transactions of the ASME, Journal of Mechanisms, Transmissions, and Automation in Design 106(4), 470-474.

322.Pisano, A. P. and F. Freudenstein (1983). An Experimental and Analytical Investigation of the Dynamic Response of a High-Speed Cam-Follower System. Part 2: A Combined, Lumped/Distributed Parameter Dynamic Model. 
Transactions of the ASME, Journal of Mechanisms, Transmissions, and Automation in Design 105(4), 699-704.

323.Pisano, A. P. and F. Freudenstein (1983). An Experimental and Analytical Investigation of the Dynamic Response of a High-Speed Cam-Follower System. Part 1: Experimental Investigation. Transactions of the ASME, Journal of Mechanisms, Transmissions, and Automation in Design 105(4), 692-698.

324.Popovici, G. A., E. Budescu, and F. Buium (1995). An Analytical Method Concerning the Unitary Synthesis of the Cam Profile. In Proceedings of the Ninth World Congress on the Theory of Machines and Mechanisms, Milan, Italy, pp. 723-726.

325.Popovic, P. and D. Domazet (1979). A Contribution to the Analysis of the Flywheel Energy Transfer of Friction Screw Presses Without Side Discs. In Proceedings of the Fifth World Congress on the Theory of Machines and Mechanisms, Montreal, Canada, pp. 453-456.

326.Potkonjak, V. and M. Vukobratovic (1983). Computer-Aided Design of Manipulation Robots Via Multi-Parameter Optimization. Mechanism and Machine Theory 18(6), 431-438.

327.Prentis, J. M. (1984). Some Personal-Computer Applications in TMM. DET Colloquium, Eindhoven, Eindhoven, Holland, August 15, 10p..[99--c] 328.Pryor, R. F., S. G. Dhande, and G. N. Sandor (1979). On The Classification and Enumeration of Six-Link and Eight-Link Cam-Modulated Linkages. In Proceedings of the Fifth World Congress on the Theory of Machines and Mechanisms, Montreal, Canada, pp. 1315-1321. 
329.Qimi, J. and Z. Ji (1996). The Design of Cam, Steel-Strip Function Mechanism. Transactions of the ASME, Journal of Mechanical Design 118(4), 532-537.

330.Ragsdell, K. M. and W. J. Carter (1975). The Energy Method. Mechanism and Machine Theory 10(2/3), 245-260.

331.Rahman, T., R. Ramanathan, R. Seliktar, and W. Harwin (1995). A Simple Technique to Passively Gravity-Balance Articulated Mechanisms. Transactions of the ASME, Journal of Mechanical Design 117(4), 655-658.

332.Rahn, C. D. and C. D. Mote, Jr. (1996). Parametric Control of Conservative Mechanical Systems. Transactions of the ASME, Journal of Dynamic Systems, Measurement, and Control 118(2), 309-314.

333.Rankers, H. (1987). Produkthandhabung in einer LichtbogenSchweiBanlage. VDI Berichte $n r$. _, 285-315.[143]

334.Rankers, H. (1984). Synthesis of Mechanisms. Computer Aided Analysis and Optimization of Mechanical System Dynamics, (incomplete).[123]

335.Rankers, H. (1983). Weg-Winkel-Zuordnung fur den KreuzschubkurbelMechanismus - Eine Exakte Kinematische MaB-Synthese-. WTHD Nr. 155, April, 12p..[121]

336.Rankers, H., A. Van Dijk, A. J. Klein Breteler, and K. Van der Werff (1976)/ 'TADSOL' - Type and Dimension Synthesis Of Link Mechanisms. Proc. Symp. Computer Aided Design in Mech. Eng., Politecnico de Milano, Italy, 51-65.[04]

337.Rankers, H. (1978). Computer Aided Design of MechanismsProgrammapakketten voor analyse en synthese van stangen-en nokmechanismen beschikbaar. Aandriftechniek, January 13.[5] 
338.Rankers, H. and et alli. (1976). 'TADSOL'-Type and Dimension Synthesis of Link Mechanisms. Proc. Symp. Computer Aided Design in Mechanical Engineering, Politecnico de Milano, Milan, October 19-21, 51-65.[101a] 339.Rankers, H. (1978). TH Delft Bedrijfsmech.(CADOM project )..[101b] 340.Rankers,H.(19..) $\geq 77)$.Computer Aided Design of Mechanical Components. [108]

341.Rankers, H. and K. M. van Buuren (1976). Computer Aided Design in Mechanical Engineering in the Netherlands in 1976. Proc. Symp. Computer Aided Design in Mechanical Engineering, Politecnico de Milano, Milan, October 19-21, 337-344.[109]

342.Rao, S. S. (1990). Mechanical Vibrations. New-York: Addison-Wesley.

343.Rao, A. C. (1983). Energy Correlation Method for the Transient Solution of a One-Degree-of-Freedom Nonlinear System. Mechanism and Machine Theory 18(5), 335-338.

344.Rao, S. S. and S. S. Gavane (1982). Analysis and Synthesis of Mechanical Error in Cam-Follower Systems. Transactions of the ASME, Journal of Mechanical Design 104(1), 52-62.

345.Rao, A. C. (1980). Synthesis of Geared Planar 4-Bar Linkages and Cams to Generate Functions of Two Variables. Mechanism and Machine Theory 15(2), 137-143.

346.Rao, A. C. (1980). Optimum Elastodynamic Synthesis of a Cam-Follower Train Using Stochastic-Geometric Programming. Mechanism and Machine Theory 15(2), 127-135. 
347.Rao, A. C. (1980). A Slotted-Crank Mechanism with a Flexibly Attached Slider for Path Generation and its Dynamic Synthesis. Mechanism and Machine Theory 15(4), 233-243.

348.Rao, A. C. (1980). Synthesis of a Mechanism with a Flexibly Attached Slider for Space Curve Generation. Mechanism and Machine Theory 15(5), 325-333.

349.Rao, J. S. and D. Y. Bibikar (1979). On The Development of the Shuttle Mechanism of the Power-Loom. In Proceedings of the Fifth World Congress on the Theory of Machines and Mechanisms, Montreal, Canada, pp. 13801383.

350.Rao, A. C. (19..)( $\geq 76)$. Four-Bars for Limited Space. Machine Design(Tech Briefs), 110, (Check the body radius for high stress), 111.[101'?]

351.Rao, J. S. and E. Raghavacharyulu (1975). Experimental Determination of Jump Characteristics in Cam-Follower Systems. J. Mech. E., 951-956.[18] 352.Reeve, J. E. (1971). Cam Indexing Mechanisms. Original Equipment Manufacture Design, October, 7p..[72]

353.Renshaw, A. A. (1998). Critical Speed for Floppy Disks. Transactions of the ASME, Journal of Applied Mechanics 65(1), 116-120.

354.Rice, S. L. (1976). 3-D Linkages Simplify Complex Mechanisms. Machine Design, June 10, 100-104.[144] 355.Ringnalda, J.et alli(?) (1992). Nokkenassen en motoren. Educatieve en technische uitgeverij Delta Press BV, The Netherlands, (it is divided in two separated blocks, both with the same order number).[178] 
356.Root, R. R. and K. M. Ragsdell (1983). The Circuit Breaker - A Practical Example in Engineering Optimization. Mechanism and Machine Theory 18(3), 229-235.

357.Rothbart, H. A. (1956). Cams. New York: John Wiley \& Sons.

358. Rothbart, H. A. (1956). Cam Dynamics of High-speed Systems. Machine Design 28(_), March 8, 100-107.[61]

359.Ruckert, H. (1980). Bewegungsgesetze fur Kurvengetriebe-Theoretische Grundlagen. VDI 2143 Blatt 1, Oktober, 27p..[87a- ]

360.Ruckert, H. (1980). Bewegungsgesetze fur Kurvengetriebe-Theoretische Grundlagen. Verein Deutscher Ingenieure(VDI), VDI 2143 Blatt 1, 27p..[85_b]

361.Ruckert, H. (1976). Bewegungsgesetz fur Kurvengetriebe mit Schwingbewegung. Antriebstechnik, 15(3), 121-124.[42]

362.Sacks, E. and L. Joskowicz (1995). Computational Kinematic Analysis of Higher Pairs with Multiple Contacts. Transactions of the ASME, Journal of Mechanical Design 117(2), 269-277.

363.Sadek, K. S. H., J. Rosinski, and M. R. Smith (1990). Natural Frequencies of a Cam Mechanism. Proc. Instn. Mech. Engrs. 204(C), 255-261.

364.Sadler, J. P., R. W. Mayne, and K. C. Fan (1980). Generalized Study of Crank-Rocker Mechanisms Driven by a d.c. Motor. Part I: Mathematical Model. Mechanism and Machine Theory 15(6), 435-445.

365.Sandgren, E. and R. L. West (1989). Shape Optimization of Cam Profiles Using a B-Spline Representation. Transactions of the ASME, Journal of Mechanisms, Transmissions, and Automation in Design 111(2), 195-201. 
366.Sandor, G. N. (1973). A General Method of Kineto-Elastodynamic Design of High Speed Mechanisms. Mechanism and Machine Theory 8(4), 497-516.

367.Savage, M. (1967). Cam Sizing Simplified. Machine Design, Oct. 26, 181185.[67]

368.Sayles, D. C. (1979). Projected Changes in the Engineer's Life-Style Within the Coming Decade. In Proceedings of the Fifth World Congress on the Theory of Machines and Mechanisms, Montreal, Canada, pp. 715-719.

369.Schaffer, G. (1974). PC plus cam = CNC. American Machinist, 118(4), 7174.[69]

370.Schumann,D.(1978). Rechnergestutzte Struktursynthese von Getriebebauformen ausgehend von konstruktiven Randbedingungen. Konstruktion,30(3), 99-106.[79]

371.Seitzer, T. (1997). Simple Cams Deliver No-Nonsense Motion Control. Machine Design, August 7, 61-67.

372.Shabana, A. (1986). Dynamics of Inertia-Variant Flexible Systems Using Experimentally Identified Parameters. Transactions of the ASME, Journal of Mechanisms, Transmissions, and Automation in Design 108(3), 358-366.

373.Shigley, J. E. (1961). Theory of Machines. New York: McGraw Hill Book Company, Inc.

374.Shiller, Z. (1996). Time-Energy Optimal Control of Articulated Systems With Geometric Path Constraints. Transactions of the ASME, Journal of Dynamic Systems, Measurement, and Control 118(1), 139-143.

375.Singer, N. C. and W. P. Seering (1990). Preshaping Command Inputs to Reduce System Vibration. Transactions of the ASME, Journal of Dynamic Systems, Measurement, and Control 112(1), 76-82. 
376.Singh, Y. P. and D. Kohli (1981). Synthesis of Cam-Link Mechanisms for Exact Path Generation. Mechanism and Machine Theory 16(4), 447-457.

377.Singhal, K. and H. K. Kesavan (1983). Dynamic Analysis of Mechanisms Via Vector Network Model. Mechanism and Machine Theory 18(3), 175-180. 378.Smith, J. A. (1995). Cams by Design. In Proceedings of the Ninth World Congress on the Theory of Machines and Mechanisms, Milan, Italy, pp. 744747.

379.Smith, J. A. (1991). A Unified Second Order Specification Algorithm for Cam Profile Synthesis. In Proceedings of the Eighth World Congress on the Theory of Machines and Mechanisms, Prague, Czechoslovakia, pp. 707-710. 380.(a)Smith, D. W. et alli. (1982). Automated Simulation and Display of Mechanism and Vehicle Dynamics. American Society of Agricultural $\begin{array}{llll}\text { Engineering, } & \text { Paper } & \text { No. } & \text { 82-5019, }\end{array}$ -(b)Rai, N. S. et alli. (1982). Computer Simulation of Suspension Abuse Tests Using ADAMS. Soc. of Automotive Engineers, Inc., Paper No.82-0079, 7p..[92-_-c]-(c)Kaufman, R. E. (1978). Mechanism Design by Computer. Machine Design, October 26, 94-100.[92-_-c = 10] (este é o mesmo artigo do número 6) -(d)Bailey, M. J. (19..). Computer Graphics in SmallScale Mechanism Design. Sandia National Laboratories, 12p..[92-_-c] -(e)Client of Mechanical Dynamics, Inc. (19..). Dynamic Analysis Capabilities and Program Features Comparison of the Integrated Mechanisms Program and the Automatic Dynamic Analysis of Mechanical Systems Program. $7 p . .[92---c]$

381.Smith, D.L. and A. H. Soni (1979). Simplified Design and Evaluation of Cam Surfaces. Forsch. Ing.-Wes. 45(1), 11-15.[7] 
382.Spector, V. A. and H. Flashner (1990). Modeling and Design Implications of Noncollocated Control in Flexible Systems. Transactions of the ASME, Journal of Dynamic Systems, Measurement, and Control 112(2), 186-193.

383.Srinivasan, L. N. and Q. J. Ge (1998). Fine Tuning of Rational B-Spline Motions. Transactions of the ASME, Journal of Mechanical Design 120(1), 46-51.

384.Srinivasan, L. N. and Q. J. Ge (1998). Designing Dynamically Compensated and Robust Cam Profiles With Bernstein-Bézier Harmonic Curves. Transactions of the ASME, Journal of Mechanical Design 120(1), 4045.

385.Stamps, F. R. and C. Bagci (1983). Dynamics of Planar, Elastic, HighSpeed Mechanism Considering Three-Dimensional Offset Geometry: Analytical and Experimental Investigations. Transactions of the ASME, Journal of Mechanisms, Transmissions, and Automation in Design 105(3), 498-510.

386.Stanescu, C. D. and C. C. Stanescu (1995). Theoretical and Technological Contributions to Kinematic of Cam Mechanics Used for Automated MachineTools. In Proceedings of the Ninth World Congress on the Theory of Machines and Mechanisms, Milan, Italy, pp. 709-712.

387.Stanescu, D. and G. Bonocoi (1979). Contributions to the Analytical Optimal Synthesis of Cams Mechanism Disk With Cam Follower Oscilatory are Employed With Automatised Machine Tools Which Use the Electronic Computer Hewlett Packard. In Proceedings of the Fifth World Congress on the Theory of Machines and Mechanisms, Montreal, Canada, pp. 1287-1290. 
388.Stanescu, D. and G. Bonocoi (1979). Contributions to the Technological Synthesis of Cams Mechanisms Disk Used With Automatised Machine Tools Which Use the Electronic Computer Hewlett Packard. In Proceedings of the Fifth World Congress on the Theory of Machines and Mechanisms, Montreal, Canada, pp. 1291-1294.

389.Stefanides, E. J. (1986). Sinusoidal Cam Engine Gives High Torque at Low RPM. Design News, 62-64.[136]

390.Stienmier, J. D., S. C. Thielman, and B. C. Fabien (1997). Analysis and Control of a Flywheel Energy Storage System With a Hybrid Magnetic Bearing. Transactions of the ASME, Journal of Dynamic Systems, Measurement, and Control 119(4), 650-656.

391.Stoddart, D. A. (1953). Polydyne Cam Design I, II, III. Machine Design, Jan., 121-135, Feb., 146-154, and March, 149-164.[54]

392.Stulen, J.H.B. (1994). Kinematica van machines en mechanismen. De Constructeur, 3, Maart, 56-59.[203]

393.Suh, C. H. (1973). Optimal Design of Mechanisms with the Use of Matrices and Least Squares. Mechanism and Machine Theory 8(4), 479-495.

394.Sutherland, G. H. (1981). Quality of Motion and Force Transmission. Mechanism and Machine Theory 16(3), 221-225.

395.Sutherland, G. H. (1978). Finding bearing loads caused by ConstantVelocity U-Joints. Machine Design, 55-59, April 20.[84a-_]

396.Sutherland, G. H. and B. Roth (1975). An Improved Least-Squares Method for Designing Function-Generating Mechanisms. Transactions of the ASME, Journal of Engineering for Industry 97(1), 303-307. 
397.Svenningsson, I. (1992). Turn-Milling. Seco Tools AB, September, 20p..[211]

398.Takano, M. and S. Toyama (1979). Dynamics of Indexing Cam Mechanism and Speed-Up of Its Motion. In Proceedings of the Fifth World Congress on the Theory of Machines and Mechanisms, Montreal, Canada, pp. 1408-1411. 399.Tascan, S. (1988). Effect of Eccentricity on the Speed Fluctuation of Cam Mechanisms With Translating Followers. Transactions of the ASME, Journal of Mechanisms, Transmissions, and Automation in Design 110(1), 11-15.

400.Terauchi, Y. and S. A. El-Shakery (1983). A Computer-Aided Method for Optimum Design of Plate Cam Size Avoiding Undercutting and Separation Phenomena - I: Design Procedure and Cycloidal Cam Design Results. Mechanism and Machine Theory 18(2), 157-163.

401.Tesar, D. ... (1991). The research program for computer controlled machines and robotics. (The University of Texas presentation to visiting commitee).[153]

402.Tesar, D. (1991). A tutorial on Modular Architecture for Robot Structures. IEEE Conference on Robotics, Sacramento, California.[154]

403.Tesar, D. and G. K. Matthew (1976). The Dynamic Synthesis, Analysis, and Design of Modeled Cam Systems. Lexington Books: D. C. Heath, Lexington, Mass.

404.Tesar, D. and G. K. Matthew (1974). The Design of Modeled Cam Systems. Mechanical Engineering, University of Florida, Gainesville, Florida, May, 66p..[75]

405.Teschler, L. (1994). Smooth moves. Machine Design, May 23, 3p..[202] 
406. Tidwell, P. H., N. Bandukwala, S. G. Dhande, C. F. Reinholtz, and G. Webb (1994). Synthesis of Wrapping Cams. Transactions of the ASME, Journal of Mechanical Design 116(2), 634-638.

407.Tiernego, M. J. L. and A. M. Bos (19..). The Dynamics and Kinematics of Mechanical Systems Modelling and Simulation With the Aid of Bond Graphs. Twente Univ. of Technology, P.O. Box 217, 7500 AE Enschede, The Netherlands, 16p..[96-_-c]

408.Ting, K.-L., G. H. Brandan, and N. L. Lee (1991). On the Selection of Control Points for Polynomial Motion Programs with Beziér Technique. In Proceedings of the Eighth World Congress on the Theory of Machines and Mechanisms, Prague, Czechoslovakia, pp. 711-714.

409. Thomopoulos, N. T. and T. W. Knowles (1975). Use of Linear Programming for Cam Design. Int. J. Mach. Tool Des. Res., 15, 257-265.[31]

410.Ting, K.-L. and Y. Long (1996). Performance Quality and Tolerance Sensitivity of Mechanisms. Transactions of the ASME, Journal of Mechanical Design 118(1), 144-150.

411.Tripathi, K. S. and K. Lakshminarayana (1995). Optimal Designs and Capability Limits of Single Roller-to-Cam Indexing Mechanisms. In Proceedings of the Ninth World Congress on the Theory of Machines and Mechanisms, Milan, Italy, pp. 727-731.

412.Tsay, D. M. and G. S. Hwang (1996). The Synthesis of Follower Motions of Camoids Using Nonparametric B-Splines. Transactions of the ASME, Journal of Mechanical Design 118(1), 138-143. 
413.Tsay, D. M. and H. M. Wei (1996). A General Approach to the Determination of Planar and Spatial Cam Profiles. Transactions of the ASME, Journal of Mechanical Design 118(2), 259-265.

414.Tsay, D. M. and G. S. Hwang (1994). Application of the Theory of Envelope to the Determination of Camoid Profiles With Translating Followers. Transactions of the ASME, Journal of Mechanical Design 116(1), 320-325.

415.Tsay, D. M. and C. O Huey, Jr.(1993). Application of Rational B-Splines to the Synthesis of Cam-Follower Motion Programs. Transactions of the ASME, Journal of Mechanical Design 115(3), 621-626.

416.Tsay, D. M. and C. O. Huey, Jr. (1988). Cam Motion Synthesis Using Spline Functions. Transactions of the ASME, Journal of Mechanisms, Transmissions, and Automation in Design 110(2), 161-165.

417.Tsai, L.-W. (1984). Oldham-Coupling Second-Harmonic Balancer. Transactions of the ASME, Journal of Mechanisms, Transmissions, and Automation in Design 106(3), 285-290.

418.Tsuji, H., H. Itoh, Y. Ogasawara, and S. Mitsuta (1995). Vibration Control by Optimal Trajectory with Feedback System (Suppression of Residual Vibration). Transactions of the Japanese Society of Mechanical Engineers, International Journal 38(4), 663-669.

419.Ullah, I. and S. Kota (1997). Optimal Synthesis of Mechanisms for Path Generation Using Fourier Descriptors and Global Search Methods. Transactions of the ASME, Journal of Mechanical Design 119(4), 504-510. 420.Václavík, M. and Z. Koloc (1977). The Simulation of Some Mechanisms on Weaving Machines. Mechanism and Machine Theory 12(6), 605-611. 
421.Van Aken, L. and H. Van Brussel (19..)( $\geq 83)$. On-Line Robot Trajectory Control in Joint Coordinates by means of Imposed Acceleration Profiles..[119]

422. Van de Straete, H. J. and J. De Schutter (1996). Hybrid Cam Mechanisms. IEEE / ASME Transactions on Mechatronics 1(4), 284-289.

423. Van den Noortgate, L. and J. De Fraine (1977). A General Computer Aided Method for Designing High Speed Cams Avoiding the Dangerous Excitation of the Machine Structure. Mechanism and Machine Theory 12(3), 237-245.

424.Van der Werff, K. (19..). Finite Element Kinematical Analysis of Spatial Mechanisms. Lab. verslagnr. 737, 7p. Department of Mechanical Engineering, Delft University of Technology, Delft, The Netherlands.[90-x-c] 425.Van Leenders, J. (1977). Automatisch gegenereerde bewerkingsplannen voor nokschijven. Metaalbewerking, 43(5), 98-103.[44] 426.VDI 2143 - Blatt 2 (1987). Bewegungsgesetze fur Kurvengetriebe Praktische Anwendung (Motion rules for cam mechanisms - Practical Application). (it is divided in two separated blocks, both with the same order number).[168]

427.Veldkamp, G. R. (1986). Oene Bottema: A Biographical Sketch. Mechanism and Machine Theory, 21(6), 447-451.[195] 428. Veron, M. (1986). Modelisation de Surfaces Complexes. Union Gramme Belgique, 25p..[207] 429.Viscomi, B. V. and R. S. Ayre (1971). Nonlinear Dynamic Response of Elastic Slider-Crank Mechanism. Transactions of the ASME, Journal of Engineering for Industry 93(1), 251-262. 
430.Von Dieter Zuck (1993). Nockenwellen- entwicklung mit SplineInterpolationen (Camshaft Development with Spline Interpolations). MTZ Motortechnische Zeitschrift, 54(12), 660-662.[179]

431.Vukobratovic, M. and N. Kircanski (1984). Computer Assisted Sensitivity Model Generation in Manipulation Robots Dynamics. Mechanism and Machine Theory 19(2), 223-233.

432.Wang, A. C. and T. W. Lee (1983). On the Dynamics of Intermittent-Motion Mechanisms. Part 2: Geneva Mechanisms, Ratchets, and Escapements. Transactions of the ASME, Journal of Mechanisms, Transmissions, and Automation in Design 105(3), 541-551.

433.Wang, A. C. and T. W. Lee (1983). On the Dynamics of Intermittent-Motion Mechanisms. Part 1: Dynamic Model and Response. Transactions of the ASME, Journal of Mechanisms, Transmissions, and Automation in Design 105(3), 534-540.

434.Wang, W. H., C. H. Tseng, and C. B. Tsay (1997). Surface Contact Analysis for a Spatial Cam Mechanism. Transactions of the ASME, Journal of Mechanical Design 119(2), 169-177.

435.Wang Y., H. Chen, and X. Shui (1995). Technical Properties and Applications of Guide Chain Transmission. In Proceedings of the Ninth World Congress on the Theory of Machines and Mechanisms, Milan, Italy, pp. 697700.

436. Weber Jr., T. (1979). Simplifying Complex Cam Design. Machine Design, March 22, 115-119.[34]

437.'Weber Jr., Theodore' (1977). Precise Cam Profiles are Generated if Computer Interpolates Equations. Product Engineering, May, 29-31.[51] 
438.Weber Jr., T. (1970). Continuous-path NC Machining of Computerdesigned Cams. Machinery and Production Engineering, March 25, 453456.[68]

439. Weber Jr., T. (1960). Cam Dynamics via Filter Theory. Machine Design, October 13, 160-165.[64]

440.Wedeniwski, H. J. (1986). Rechnergestutzte Programmierung beim CNCNockenform-Schleifen. Werkstatt und Betrieb 119(8), 655-660.[137]

441.West, M. and H. Asada (1997). Design of Ball Wheel Mechanisms for Omnidirectional Vehicles With Full Mobility and Invariant Kinematics. Transactions of the ASME, Journal of Mechanical Design 119(2), 153-161.

442.Westinghouse' (1975). Cost Effective measurement: Simplified Cam Checking. Metrology and Inspection, Dec., 25-26.[41]

443.Wiederrich, J. L. (1983). A Theory for the Identification of a Single Degreeof-Freedom Machine From its Periodic Operating Characteristics. Transactions of the ASME, Journal of Mechanisms, Transmissions, and Automation in Design 105(3), 445-451.

444.Wiederrich, J. L. and B. Roth (1976). Momentum Balancing of Four-Bar Linkages. Trans. ASME, J. Eng. for Ind., paper no. 76-det-28, 1-7.[38] 445.Wiederrich, J. L. and B. Roth (1975). Dynamic Synthesis of Cams Using Finite Trigonometric Series. Transactions of the ASME, Journal of Engineering for Industry 97(1), 287-293.

446.Wiederrich, J. L. and B. Roth (1974). Dynamic Synthesis of Cams Using Finite Trigonometric Series. Trans. ASME, J. Eng. for Ind., p. no.74-det-2. 17.[39] 
447.Winfrey, R. C., R. V. Anderson, and C. W. Gnilka (1973). Analysis of Elastic Machinery With Clearances. Transactions of the ASME, Journal of Engineering for Industry 95(3), 695-703.

448.Wodzicki, W. (1979). Dynamic Characteristic of Passive and Active Flexible Objects. In Proceedings of the Fifth World Congress on the Theory of Machines and Mechanisms, Montreal, Canada, pp. 289-292.

449.Xu, H. D. (1994). Motion Conversion by Means of Perturbation of Elastic Field. Transactions of the ASME, Journal of Mechanical Design 116(1), 232237.

450. Yamada, I. and M. Nakagawa (1985). Reduction of Residual Vibrations in Positioning Control Mechanism. Transactions of the ASME, Journal of Vibration, Acoustics, Stress, and Reliability in Design 107(1), 47-52.

451.Yan, H.-S., M.-C. Tsai, and M.-H. Hsu (1996). A Variable-Speed Method for Improving Motion Characteristics of Cam-Follower Systems. Transactions of the ASME, Journal of Mechanical Design 118(2), 250-258.

452.Yan, H.-S. and H.-H. Chen (1996). Geometry Design of Globoidal Cams With Generalized Meshing Turret-Rollers. Transactions of the ASME, Journal of Mechanical Design 118(2), 243-249.

453.Yan-ming, F., L. Xian-li, and Y. Hong-liang (1995). Design of Conjugate Indexing Disk Cam Mechanism. In Proceedings of the Ninth World Congress on the Theory of Machines and Mechanisms, Milan, Italy, pp. 701-705.

454.Yoon, K. and S. S. Rao (1993). Cam Motion Synthesis Using Cubic Splines. Transactions of the ASME, Journal of Mechanical Design 115(3), 441-446. 
455.Young, S.-S. D. and T. E. Shoup (1982). The Sensitivity Analysis of Cam Mechanism Dynamics. Transactions of the ASME, Journal of Mechanical Design 104(2), 476-481.

456.Yu, P. (1997). Explicit Vibration Solutions of a Cable Under Complicated Loads. Transactions of the ASME, Journal of Applied Mechanics 64(4), 957964.

457.Yu, Q. and H. P. Lee (1995). A New Family of Parametrized Polynomials for Cam Motion Synthesis. Transactions of the ASME, Journal of Mechanical Design 117(4), 653-655.

458.Zadoks, R. I. and A. Midha (1987). Parametric Stability of a Two-Degree-ofFreedom Machine System: Part II - Stability Analysis. Transactions of the ASME, Journal of Mechanisms, Transmissions, and Automation in Design 109(2), 216-223.

459.Zadoks, R. I. and A. Midha (1987). Parametric Stability of a Two-Degree-of-

Freedom Machine System: Part I - Equations of Motion and Stability.

Transactions of the ASME, Journal of Mechanisms, Transmissions, and Automation in Design 109(2), 210-215.

460.Zahradka, J. (1978). An Analysis of Self-excited Vibrations where the Effects of Machine Characteristics are Considered. Mechanism and Machine Theory 13(1), 57-73.

461.ZhiSu, Z. (1995). On Design Method of the Precision Cam Profile with Random Processing Errors. In Proceedings of the Ninth World Congress on the Theory of Machines and Mechanisms, Milan, Italy, pp. 751-753.

462.Zsombor-Murray, P. J. (1991). A Mechanism to Generate a SpheroCylindrical Trace. Abstract for 5th Int. Conf. on Eng. Comp. Graph. \& 
Descriptive Geometry, 3p., Melbourne, Australia,

-- Zsombor-Murray, P. J. (1991). Quadratic Surface Intersections (Trace Modeling \& Identification of Parametric Limits).[161]

463.(ADAMS) Mechanical Dynamics, Inc., Engineering Software and Services (ADAMS), Adresses[139]

464.(ADAMS) McConville, J. (1987). An Elastic Approach for Large Displacement Dynamics. Computers in Mechanical Engineering(CIME), 5(5), March.[140]

465.ADAMS, Quality Engineering Software(About ADAMS...)[141]

466.(ADAMS and Stefanides) Stefanides, E. J. (1985). General Simulation Program Prototypes Fruit Juicer. Design News, 3p..[142] 
ANEXO A - Regularização da Velocidade com um Mecanismo Inercial Adaptativo por Compensação de Torque (Ballerina Flywheel) 


\section{Regularização da Velocidade por Compensação de Torque com um Mecanismo Inercial Adaptativo (Ballerina Flywheel)}

Luiz Augusto Martin Gonçalves ${ }^{a}$, Jeferson Cassiano ${ }^{b}$, José Manoel Balthazar ${ }^{c}$

Este trabalho diz respeito à análise do comportamento dinâmico de um mecanismo inercial adaptativo para a regularização de velocidade por compensação de torque (Ballerina Flywheel) de forma a se compreender o fenômeno da regularização de velocidade com vistas à sua aplicação em problemas de engenharia tais como o que foi objeto desta tese, onde a não estabilidade da velocidade impedia o sistema de came e seguidor com compensação por pré-alimentação apenas da dinâmica linear do sistema seguidor de efetuar corretamente a ação para ele projetada. 


\section{Introdução}

No que diz respeito a problemas de engenharia sabe-se que as vibrações lineares tem sido exaustivamente estudadas nos últimos anos. Já o estudo das vibrações não lineares tem tido algum progresso nesse mesmo período.

Vibrações ideais são aquelas que não apresentam interações entre a fonte de energia e o problema físico [Balthazar et al., 2003] and [Balthazar et al., 2004].

Aqui tratamos de ambas, vibrações lineares e não lineares de um problema mecânico chamado de regularização de velocidade com a utilização de um mecanismo adaptativo para regularização de velocidade por compensação de torque. Esta propriedade é comum em algumas máquinas rotativas [Tondl, 1965]; [Rao, 1983]; [Genta, 1995].

Deve-se enfatizar que o estudo deste assunto deve ser feito envolvendo o conhecimento das dinâmicas linear e não linear do dispositivo adaptativo de compensação em questão. Ele pode ser modelado tanto física como matematicamente, a partir do mecanismo descrito na figura 1. 
Na figura 1, temos as seguintes notações:

$\Omega=$ Constant; $\theta=\Omega t+\alpha ; l=$ free length of $k ; r=r_{0}+\xi ; r_{0}=1+\delta ; \delta=$ Initial deflection; $\xi=$ dynamic deflection; $\delta=\delta_{\text {constan } t \text { speed }}+\delta_{\text {static }}$.

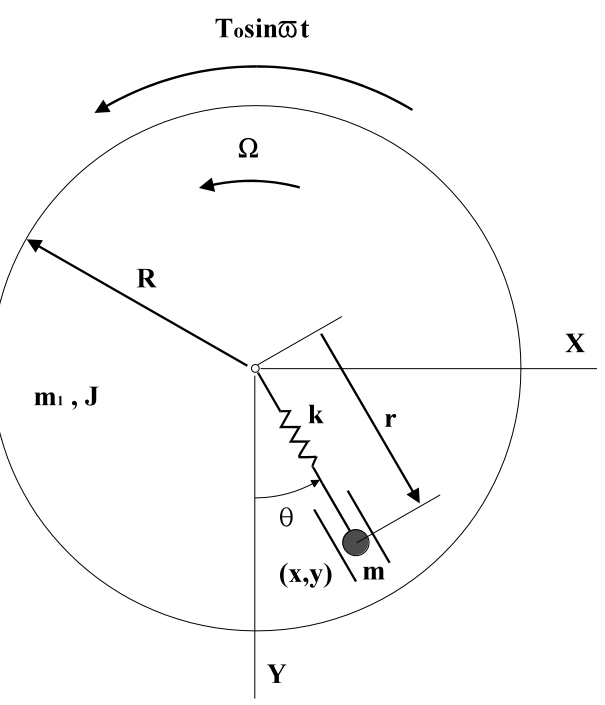

Fig 1: Modelo Físico do Mecanismo Inercial Adaptativo de Regularização de Velocidade por Compensação de Torque (ballerina flywheel)

É de se notar que a excitação tem amplitude constante $T_{0}$ e freqüência de excitação $\omega$.

O objetivo deste trabalho é analisar o comportamento das vibrações ideais tanto lineares como não lineares para o problema mostrado na figura 1. Podem ser observadas diferenças significativas nesse comportamento que devem ser consideradas pelos projetistas em casos onde se aplicam. 
Na seção 2 são discutidas a derivação das equações de movimento e também apresentadas algumas propriedades relacionadas ao comportamento dinâmico linear e não linear para os modelos matemáticos considerados. $\mathrm{Na}$ seção 3 são apresentadas as conclusões seguidas de alguns agradecimentos e das referências bibliográficas utilizadas para este trabalho.

\section{Obtenção das Equações de Movimento}

As expressões para a energia cinética $T$ e para a energia potencial $U$ para o sistema definido pela figura 1 são dadas por

$$
T=\frac{1}{2} J \dot{\theta}^{2}+\frac{1}{2} m\left(\dot{r}^{2}+r^{2} \dot{\theta}^{2}\right)
$$

e

$$
U=\sum_{i=1}^{4} U_{i}
$$

com

$$
\begin{gathered}
U_{1}=\int_{0}^{\xi} k(\xi+\delta) d \xi+m g\left(r_{0}+\left(r_{0}+\xi\right) \cos \theta,\right. \\
U_{2}=\frac{1}{2} k\left(r_{0}+\xi-l\right)^{2}+m g\left[r_{0}-\left(r_{0}+\xi\right) \cos \theta\right] \\
U_{3}=\frac{1}{2} k(\xi+\delta)^{2}+m g\left[r_{0}-\left(r_{0}+\xi\right) \cos \theta\right] ; \\
U_{4}=\frac{1}{2} k \xi^{2}+\frac{1}{2} k \delta_{s t}^{2}+k\left(\delta_{\pi}+\delta_{s t}\right) \xi+m g r_{0}-m g r \cos \theta
\end{gathered}
$$

Deve-se notar que o termo $k \delta$ representa a deflexão inicial da mola $k$. Em um movimento horizontal, sem efeitos gravitacionais, $k \delta=k \delta_{c s}=k \delta_{\text {constan tspeeed }}$ que significa que o sistema vibratório está em equilíbrio antes da aplicação da 
força de distúrbio. Se o sistema estiver posicionado de forma a operar em um plano vertical onde os efeitos gravitacionais tem que ser considerados, então isso resulta em $k \delta=k \delta_{c s}+k \delta_{s t}=k \delta_{c s}+m g$.

Desta forma a função Lagrangiana é $L=T-U$. Com a utilização das equações de Lagrange de movimento obtém-se

$$
\left\{\begin{array}{l}
\varangle+m r^{2} \vec{\theta}+2 m r \dot{r} \dot{\theta}+m g r \operatorname{sen} \theta+T_{0} \operatorname{sen} \measuredangle t=0 \\
m \ddot{r}-m r \dot{\theta}^{2}+k \varangle-r_{0}+k \delta_{c s}+m g(-\cos \theta=0
\end{array}\right.
$$

Para se obter uma perfeita compensação entre o torque de distúrbio e o movimento do sistema massa mola sob investigação, duas condições devem ser satisfeitas:

I-) o sistema como um todo deve ter $\alpha=\dot{\alpha}=\ddot{\alpha}=0 \quad \mathrm{e}$

II-) a freqüência natural do sub-sistema massa mola deve ser igual à freqüência de distúrbio $\omega$.

As equações (1) podem então ser escritas como

$\left(+m r_{0}^{2} \stackrel{\ddot{\alpha}}{ }+2 m r_{0} \Omega \dot{\xi}+m\left(2 r_{0}+\xi\right) \xi \ddot{\alpha}+2 m\left(\Omega \xi+r_{0} \dot{\alpha}+\xi \dot{\alpha}\right) \dot{\xi}+m g\left(r_{0}+\xi\right) \sin (\Omega t+\alpha)+\right.$ $+T_{0} \operatorname{sen} \varphi 0$

$m \ddot{\xi}+\left(k-m \Omega^{2}\right) \xi-2 m r_{0} \Omega \dot{\alpha}-m\left(\left(r_{0}+\xi\right) \dot{\alpha}^{2}+2 \Omega \xi \dot{\alpha}\right)-m g \cos (\Omega t+\alpha)=0$

A seguir discute-se algumas propriedades do comportamento dinâmico linear.

\subsection{Comportamento Dinâmico Linear}

Linearizando-se as equações e desprezando-se os termos gravitacionais obtém-se: 


$$
\begin{aligned}
& \left(+m r_{0}^{2} \ddot{\ddot{g}}+2 m r_{0} \Omega \dot{\xi}+T_{0} \operatorname{sen} \varphi t=0\right. \\
& m \ddot{\xi}+\left(k-m \Omega^{2}\right) \xi-2 m r_{0} \Omega \dot{\alpha}=0
\end{aligned}
$$

De forma a satisfazer as condições I-) e II-) acima nós obteremos a partir da primeira equação 1b-) que $\frac{k}{m}=\omega^{2}+\Omega^{2}$. Note também que esta solução é $\xi=\Sigma \cos \omega t$ onde $\Sigma$ é a máxima amplitude de $\xi$. Para a segunda equação de 1b-) nós obteremos que $\Sigma=\frac{T_{0}}{2 m r_{0} \Omega \omega}$.

Dado que os efeitos gravitacionais se apresentam de forma harmônica, eles podem ser facilmente compensados com a força ativa aplicada por um atuador. A relação obtida anteriormente não é exata dado que foi obtida a partir de equações linearizadas, mas é uma razoável aproximação. Uma vez que se escolha um valor para a massa, a rigidez da mola fica então diretamente determinada ou, ao contrário, a escolha da rigidez da mola impõe o valor da massa. O único valor que deve ainda ser determinado antes de se aplicar a relação é $r_{0}$ que pode ser determinado a partir de $-m \Omega^{2} r_{0}+k \delta=0$ onde $r_{0}=l+\delta$

A seguir, discute-se as propriedades dinâmicas não lineares.

\subsection{Comportamento Dinâmico Não Linear}

Assumindo que $\left\{\tau=\omega t, x_{3}=r / r_{0}, \alpha=g /\left(\omega^{2} r_{0}\right), \beta=T_{0} /\left(\omega^{2} \mathrm{mr}_{0}^{2}\right), \gamma=\mathrm{J} /\left(\mathrm{mr}_{0}^{2}\right), \rho=\right.$ $\left.\mathrm{k} /\left(\omega^{2} \mathrm{~m}\right), \delta=\delta_{\mathrm{cs}} / \mathrm{r}_{0}, \mathrm{x}_{1}=\theta, \mathrm{x}_{2}=\dot{\theta} / \omega \mathrm{e} \mathrm{x}_{4}=\dot{\mathrm{x}}_{3} / \omega\right\}$

então o campo vetorial pode ser dado por 


$$
\mathbf{f}: \nabla^{3} \times S \rightarrow T\left(\nabla^{3} \times S\right), \mathbf{x} \mapsto\left[\begin{array}{c}
\mathrm{x}_{2} \\
-\left(\begin{array}{c}
2 \mathrm{x}_{2} \mathrm{x}_{3} \mathrm{x}_{4}+\alpha \mathrm{x}_{3} \operatorname{sen} \mathrm{x}_{1}+\beta \operatorname{sen} \tau \\
\gamma+\mathrm{x}_{3}^{2} \\
\mathrm{x}_{4}
\end{array}\right) \\
\mathrm{x}_{2}^{2} \mathrm{x}_{3}-\rho \mathrm{s}_{3}-1-\alpha<-\cos \mathrm{x}_{1}-\rho \delta
\end{array}\right]
$$

Se considerarmos que $\beta=0$, obtém-se então um sistema Hamiltoniano no qual a função hamiltoniana é dada por

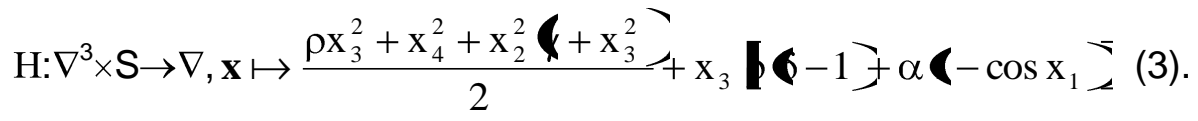

Então, o campo vetorial pode ser expresso pela seguinte expressão

$$
\mathbf{f}_{0}: \nabla^{3} \times \mathrm{S} \rightarrow T\left(\nabla^{3} \times \mathrm{S}\right), \quad \mathbf{x} \mapsto\left[\begin{array}{c}
\mathrm{x}_{3} \boldsymbol{\phi}+\mathrm{x}_{2}^{2} \neq \rho-1 \neq \alpha\left(-\cos \mathrm{x}_{1}\right] \\
\mathrm{x}_{4} \\
-\alpha \mathrm{x}_{3} \operatorname{sen} \mathrm{x}_{1} \\
-\mathrm{x}_{2}\left(+\mathrm{x}_{3}^{2}-\right.
\end{array}\right]
$$

Os pontos fixos do sistema são:

$$
\begin{aligned}
& \mathbf{x}_{1}=\left[\begin{array}{lllll}
0 & 0 & 1-\delta & 0
\end{array}\right]^{t} \mathrm{e} \\
& x 2=[\arccos [1-(\rho / \alpha)(1-\delta)] \quad 000] .
\end{aligned}
$$

Os autovalores do Jacobiano obtidos pelo uso do sistema linear associado são

$$
\begin{array}{ll}
\text { para x1 } & \pm \mathrm{j} \sqrt{\gamma+\left(-1^{\gamma}\right.} ; \pm \sqrt{\rho \alpha-1}, \mathrm{j}^{2}=-1 \quad \mathrm{e} \\
\text { para x2 } & \pm j \sqrt{\gamma} ; \pm j \sqrt{\rho \alpha \boldsymbol{\phi}-1)} \alpha+\rho(-1) .
\end{array}
$$

Há dois casos a se considerar: $\delta>1$ onde a matriz Jacobiana $\mathbf{x}_{2}$ tem todos os autovalores imaginários puros e em $\mathbf{x}_{1}$ ela tem dois autovalores reais e com sinais opostos; $\delta<1$ onde ocorre justamente a situação oposta. De acordo com 
as propriedades Hamiltonianas, nós sabemos que em cada caso ela tem para um dos pontos um manifoldo sub-central e para um outro ponto ela pode ser decomposta em um sub-manifoldo que pode então ser decomposto em ambos em expanão e em contração.

De forma a se estudar a natureza da estabilidade dos pontos fixos não hiperbólicos necessita-se usar a teoria dos manifoldos centrais [Car, 1981], [Guckheimer \& Holmes, 1985]; [Wiggins, 1980]; [Thompson \& Bishop, 1994]; [Nayfeh \& Balachandran, 1994), entre outros. Então, no primeiro caso, fazendose apropriadas mudanças de variáveis pode-se obter

$$
\begin{aligned}
& \frac{d}{d t}\left[\begin{array}{l}
x_{1} \\
x_{3}^{\prime}
\end{array}\right]=\left[\begin{array}{cc}
0 & \rho \\
\alpha \boldsymbol{\sigma}-1 & 0
\end{array}\right]\left[\begin{array}{l}
x_{1} \\
x_{3}^{\prime}
\end{array}\right]+\left[\begin{array}{c}
x_{2}^{2}+1-\delta+\alpha\left(-\cos x_{1}\right. \\
\alpha-\delta-\delta \operatorname{sen} x_{1}
\end{array}\right]
\end{aligned}
$$

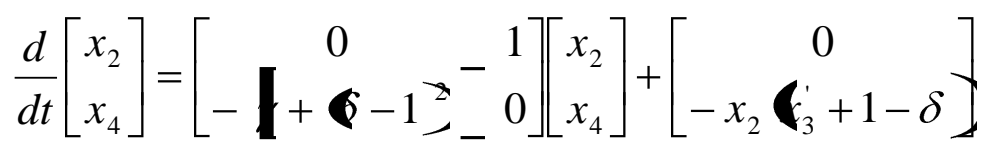

e as condições intrínsicas são obtidas através de soluções pelas seguintes equações diferenciais a derivadas parciais com as seguintes condições de

$$
\begin{aligned}
& \text { contorno } x 1(0)=x_{2}(0)==\frac{\partial x_{1}}{\partial x_{2}}=\frac{\partial x_{3}^{\prime}}{\partial x_{2}} \varphi_{-}=0
\end{aligned}
$$

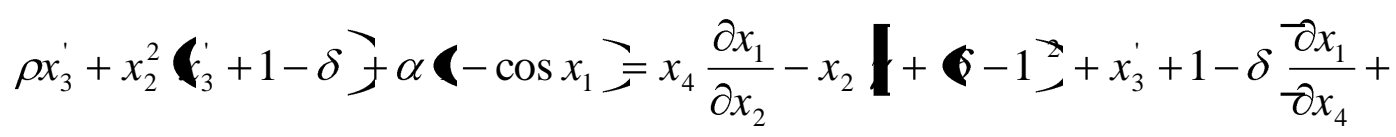

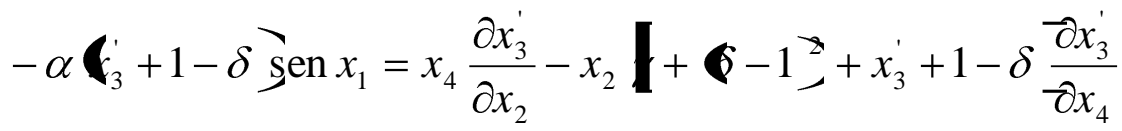

A solução analítica desta equação é complexa mas pode ser escrita na forma polynomial, ao menos localmente [Carr, 1981]:

$$
\mathrm{x}_{1}=\sum_{\mathrm{i}=0}^{2} \mathrm{a}_{\mathrm{i}} \mathrm{x}_{2}^{\mathrm{i}} \mathrm{x}_{4}^{2-\mathrm{i}}+\sum_{\mathrm{j}=0}^{3} \mathrm{~b}_{\mathrm{j}} \mathrm{x}_{2}^{\mathrm{j}} \mathrm{x}_{4}^{3-\mathrm{j}}+\mathrm{o}
$$


$\mathrm{x}_{3}^{\prime}=\sum_{\mathrm{m}=0}^{2} \mathrm{c}_{\mathrm{m}} \mathrm{x}_{2}^{\mathrm{m}} \mathrm{x}_{4}^{2-\mathrm{m}}+\sum_{\mathrm{n}=0}^{3} \mathrm{k}_{\mathrm{n}} \mathrm{x}_{2}^{\mathrm{n}} \mathrm{x}_{4}^{3-\mathrm{n}}+\mathrm{o}$

Se se substitui então em (6), obtém-se que a central é dada por

$W^{c}=\left\{\left(x_{1}, x_{2}, x_{3}{ }^{\prime}, x_{4}\right) \in \nabla^{3} \times S: x_{1}=a_{1} x_{2} x_{4}+o(4), x_{3}{ }^{\prime}=c_{0} x_{4}{ }^{2}++c_{2} x_{2}{ }^{2}+o(4)\right\}$

com

$a_{1}=\frac{2(-1)}{8-12 \delta+4 \delta^{2}+4 \gamma-\alpha \rho+\alpha \rho \delta}$

$\mathrm{c}_{0}=\frac{\mathrm{a}_{1}}{\rho}$,

$c_{2}=\frac{\phi-1}{\rho}\left\lceil 1-\frac{1+(-1)-\delta+\gamma}{8-12 \delta+4 \delta^{2}+4 \gamma-\alpha \rho+\alpha \delta \rho}\right]$

Então, o sistema vibratório sob Wc pode ser escrito como

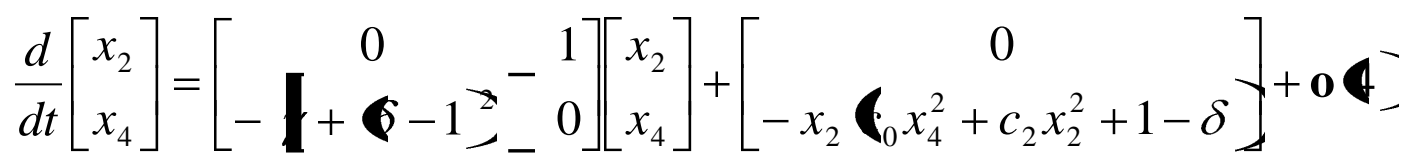

Nota-se que o sistema (10) é instável.

\section{Conclusões}

As equações foram linearizadas, mas isto é uma aproximação razoável para

o estudo da regularização de velocidade com um mecanismo inercial adaptativo por compensação de torque (ballerina flywheel); as equações não lineares para o problema podem ser escritas na forma Hamiltoniana. Elas exibem as características de instabilidade do problema.

Este trabalho mostra os distintos comportamentos dinâmicos nos casos em que se considera vibrações lineares e vibrações não lineares.

Em trabalhos futuros consideraremos a influência da fonte de energia (por exemplo um pequeno motor DC com limitações na potência disponível) na vibração de um dispositivo como o aqui considerado para se equacionar este 
problema de vibração em um sistema não ideal [Balthazar et al., 2003]; and [ Balthazar et al., 2004].

Deseja-se agradecer à Fundação de Amparo à Pesquisa do Estado de São Paulo - FAPESP e ao Conselho Nacional de Pesquisas -CNPq pelo suporte financeiro.

\section{Referências}

Balthazar, J.M., Brasil, R.M.L.R.F., Weber.H.I., Fenili, A., Belato, D., Felix, J.L.P. and Garzelli F.J. [2004], “ A Review of New Vibration Issues due to Non-Ideal Energy Sources “, in Udwadia, F.E., Webwer, H.I. and Leitmann, G. (eds), Dynamical Systems and Control, Stability and Control: Theory, Methods and Applications 22, Chapman \& Hall/ CRC, Boca Raton, Balthazar, J. M., Mook, D. T., Weber, H. I., Reyolando, M. L. R. F., Fenili, A., Belato \& D., Felix, J.L. P, [2003]. "An overview on non-ideal vibrations". Meccanica, 3 (5), 493- 503.

Carr, J., [1981], “Applications of Center Manifold Theory”. Springer-Verlag, New York, Heidelberg, Berlin, 1981.

Genta G [1995], “ Vibration of structures and machines “, Springer- Verlag, 1995

Guckenheimer, J. \& Holmes, P., [1985],' “Nonlinear oscillations, dynamical systems and Bifurcations of vector fields", Springer-Verlag, New York. 
Nayfeh, A. H. and Balachandran, B., [1995], "Applied nonlinear dynamics", John Wiley \& Sons, New York.

Rao, J S, [1983], “ Rotor dynamics”, Wiley Eastern Limited

Thompson JMT \& Bishop SR, [1994], "Nonlinearity and Chaos in Engineering Dynamics", John Wiley \& Sons.

Tondl, A, [1965]," Some Problems of Rotor Dynamics", Chapman \& Hall Limited.

Wiggins, S., [1980], "Global Bifurcation and Chaos. Applied Mathematical Sciences " (Volume 73). Springer Verlag. 